\title{
A hybrid moment of fluid-level set framework for simulating primary atomization
}

\author{
Anirudh Asuri Mukundan ${ }^{\mathrm{a}, 1}$, Thibaut Ménard ${ }^{\mathrm{a}, \mathrm{b}, *}$, Jorge César Brändle de Motta $^{\mathrm{a}, \mathrm{b}}$, Alain Berlemont ${ }^{\mathrm{a}}$ \\ ${ }^{a}$ CNRS UMR6614 CORIA, Saint-Étienne-du-Rouvray, France \\ ${ }^{b}$ Université de Rouen Normandie, Saint-Étienne-du-Rouvray, France
}

\begin{abstract}
This paper presents a hybrid moment of fluid-level set (HyMOFLS) method of liquid/gas interface reconstruction for the application to simulate primary atomization of liquid fuel. This method combines the moment of fluid (MOF) method and the level set framework in the coupled level set volume of fluid (CLSVOF) method. In this hybrid method, the MOF and CLSVOF methods are used to reconstruct the interface in the under-resolved and the resolved regions of the flow, respectively. An interface surface resolution metric called interface resolution quality (IRQ) is introduced to identify and classify these two flow regions in the computational domain. Such a strategy classifies/tags each computational cell with MOF or CLSVOF method based on a threshold value for the IRQ. The MOF method uses liquid volume fraction as well as centroids of liquid and gas phases for the interface reconstruction. The CLSVOF method uses the level set for describing the interface and liquid volume fraction for conserving the mass. The phase centroids in the HyMOFLS method are computed and transported on-the-fly during the cell tagging process. The transport of the liquid volume fraction, level set, and the phase centroids are performed using a directionally split algorithm. This algorithm is coupled with the Navier-Stokes equations solver that uses ghost fluid method as well as consistent mass and momentum flux computation for the momentum equation. Various numerical tests that exhaustively assess the capabilities, accuracy, and computational time consumption of the HyMOFLS method under multiple flow conditions and configurations are presented. The results from these tests suggests that this hybrid framework is capable of well capturing the liquid/gas interface belonging to thin and under-resolved structures that are often encountered in simulations of turbulent atomization of liquids. Following these tests, a detailed parametric study on the threshold value of IRQ is presented to test its effect on the accuracy of interface reconstruction. Finally, this hybrid framework is employed to simulate turbulent jet injection and pre-filming planar Airblast atomziation cases of engineering applications. The proposed HyMOFLS method is found to achieve a good balance between the accuracy and the computational cost of reconstructing the liquid/gas interface for complex and turbulent atomization configurations.
\end{abstract}

Keywords: DNS, Primary atomization, Incompressible flow, Multiphase flow, Moment of Fluid method, Level set method

\section{Motivation and objectives}

The numerical simulations of primary atomization of liquid fuel provide insights into the understanding of the droplet breakup. With the increase in the computational power over the past decades, it has become

\footnotetext{
${ }^{*}$ Corresponding author

Email addresses: anirudh.mukundan@math.ubc.ca, anirudh.mukundan@coria.fr (Anirudh Asuri Mukundan ), thibaut.menard@coria.fr (Thibaut Ménard)

${ }^{1}$ current address: Department of Mathematics, University of British Columbia, Vancouver, Canada. Contact Email address: anirudh.mukundan@math.ubc.ca
} 
possible to perform detailed numerical simulations of primary atomization process [1] on large scale geometries [2]. The atomization process is turbulent, multiphase (liquid and gas phases), multi-scale (varying drop sizes), and multi-physics (presence of various physical events such as Rayleigh-Taylor, Kelvin-Helmholtz, and Rayleigh-Plateau instabilities) in nature. The simulation of such a complex process requires accurate numerical methods and schemes that can handle large discontinuities across liquid/gas interface, jump in pressure and viscosity, singular nature of the surface tension force acting on the location of the interface, and finally, accurate description and transport of the liquid/gas interface.

Two of the most prominent methods of handling the discontinuities in the material properties across the interface are continuum surface force (CSF) model [3] and ghost fluid method (GFM) [4. These methods depend on the numerical methods responsible for the description and transport of the interface. The prominent classes of methods for this purpose are the volume of fluid (VOF) method [5, 6, 7] and the level set (LS) method [8, 9, 10. The former tracks the liquid volume fraction thereby ensuring mass conservation while the latter tracks the interface in the form of the iso-contour of a signed distance level set function thereby computing accurate geometrical properties of the phase interface. There have been many improvements made over the years for the VOF method [11, 12, 13, 14] and LS method [15, 10, 16. A combined strategy of coupled level set volume of fluid (CLSVOF) method [17, 18, 19, exploits the advantage of both the VOF and LS methods. This method has been successful in simulating liquid fuel primary atomization process as shown by Ménard et al. 18. Another type of interface tracking and reconstruction includes the refined level set grid (RLSG) method 20] which locally refines the LS mesh to control the errors arising from the interface transport and to compute the interface curvature accurately. Yet another approach of interface reconstruction was presented by Desjardins and Pitsch 21 using a spectrally refined interface (SRI) approach in which a polynomial reconstruction of the LS function is created in each computational cell leading to higher accuracy of the small scale transport.

In order to well understand the primary atomization, it is necessary to capture the small and often underresolved liquid structures (URLS). When such liquid structures are of the size of the computational mesh spacing, it can lead to inaccuracies in the computation of the interface geometrical properties and in the reproduction of physics of droplet breakup. The recently developed moment of fluid (MOF) method 22, 23. 24, 25, 26 of liquid/gas interface reconstruction specifically addresses this aspect. This method uses liquid volume fraction along with centroids of liquid and gas phases in each computational cell to reconstruct the interface. This approach simultaneously conserves the volume and preserves the accurate orientation of the interface. It has been shown 27] that the MOF method yields relatively low interface reconstruction errors and higher order of grid convergence of this error. There have been various implementations and extensions made in the recent past to the original MOF method [22, such as, the analytical interface reconstruction [28, 29] in two dimensions [30, 31] which was then extended to three dimensions [32, extension to compressible flow simulations 33], accurate capture of the thin filaments [34], two-plane interface reconstruction [35], and to many other applications and grid types [36, 37, 38, 39, 40, 41, 42, 43, 44.

In a recent study, Asuri Mukundan et al. [45] compared the MOF and CLSVOF methods for variety of tests and found that the MOF method outperformed the CLSVOF method in terms of accuracy of interface reconstruction. However, the MOF method was found to be computationally expensive than the CLSVOF method in simulating primary atomization simulations 46. Naturally, a numerical method having high accuracy and modest computational cost requirement is required to be employed for simulating large scale applications. Thus, to that end, we have developed in this work a hybrid moment of fluid-level set (HyMOFLS) method as a combination of MOF and CLSVOF methods. The coupling between these two methods is solely in choosing the way the interface reconstruction need to be made with a choice between MOF-based and level set-based method. The rationale behind the development of HyMOFLS method is to use the MOF method in the computational domain only when it is necessary while the CLSVOF method almost all the time for interface reconstruction. The necessity is driven by the presence of URLS in the computational domain. The obvious question at this juncture is, how to identify under-resolved regions of the flow? To answer that question, a metric for interface surface resolution called interface resolution quality (IRQ) [47] is employed in this work that classifies and distinguishes the under-resolved from the resolved flow regions. A similar criterion has been been employed before by Jemison et al. 27] in their simulations. Based on the threshold value for the IRQ criterion, a computational cell is classified/tagged as to whether it 
belongs to URLS or not; and by extension, a decision whether MOF method 45 is necessary to reconstruct the interface in that cell or not.

This paper is organized as follows. Section 2 presents the framework of the HyMOFLS method implemented in our solver followed by the canonical tests including Zalesak's notched disk, 2D, and 3D droplet deformation comparing the results between the HyMOFLS, MOF, and CLSVOF methods. Then, a detailed parametric study on the threshold value of the IRQ criterion is presented in which the optimal value is found such that a balance exist between the accuracy and computational costs for the HyMOFLS method. Section 3 presents the solution procedure employed to solve the Navier-Stokes equations along with the discretization of various terms and time integration scheme employed in our flow solver. The numerical validation tests are presented in Section 4 to assess the capabilities of the hybrid framework for a convection dominated two-phase shear layer, Rayleigh-Taylor instability, binary droplet collision case, and RayleighPlateau instability. Finally, Section 5 presents the results from the numerical simulations of engineering applications - turbulent jet atomization under diesel engine-like condition and planar pre-filming Airblast atomization using the HyMOFLS method.

\section{Hybrid MOF-Level set (HyMOFLS) method}

This section describes in detail the hybrid moment of fluid-level set (HyMOFLS) approach for liquid/gas interface reconstruction implemented in our in-house Navier-Stokes equations solver ARCHER [48]. The HyMOFLS method involves the coupling between the moment of fluid (MOF) method 45 and the coupled level set volume of fluid (CLSVOF) method [18. On one hand, the MOF method has been shown [45] to have higher order accuracy especially in capturing under-resolved liquid structures (URLS) such as thin ligaments and small droplets; however, incurs high computational cost. On the other hand, the CLSVOF method yields lesser computational cost 18 by reaching relatively lower order of accuracy. The motivation for the development of the HyMOFLS method stems from the requirement of increased accuracy to be achieved at a modest computational expense by coupling MOF and CLSVOF methods. The rationale to couple the more accurate MOF method with the level set framework is to use the former for capturing the interface of under-resolved flow regions while the latter for resolved flow regions of the computational domain. The presence of the under-resolved regions in the computational domain is determined using local mesh spacing $\Delta x$ and the local interface curvature $\kappa$ (see Section 2.3 for more details on coupling procedure). A similar method for interface reconstruction has been explored by Jemison et al. 27] in the past. The description of the CLSVOF and MOF methodologies along with the HyMOFLS coupling procedure are presented in the following subsections.

\subsection{Coupled level set volume of fluid (CLSVOF) method}

\subsubsection{Level set}

The level set methods [9, 49, 50] use a contiguous signed distance function $\phi(x, t)$ to describe the location of the interface between two phases where $\boldsymbol{x}$ and $t$ represent the spatial location and time instant, respectively. The value of $\phi(\boldsymbol{x}, t)>0$ defines the liquid phase, $\phi(\boldsymbol{x}, t)<0$ defines the gas phase, and $\phi(\boldsymbol{x}, t)=0$ defines the location of the liquid/gas interface. The signed distance represent the distance between any point in the computational domain to the interface.

The geometrical properties of the interface are directly computed from the level set function $\phi$. For example, the unit normal of the interface is computed as

$$
\boldsymbol{n}=\frac{\boldsymbol{\nabla} \phi}{\|\boldsymbol{\nabla} \phi\|_{2}} .
$$

The curvature of the interface is then computed as:

$$
\kappa=-\boldsymbol{\nabla} \cdot \boldsymbol{n}=-\boldsymbol{\nabla} \cdot\left(\frac{\boldsymbol{\nabla} \phi}{\|\boldsymbol{\nabla} \phi\|_{2}}\right),
$$


It is to be remarked that the interface unit normal points towards the liquid phase and the curvature is positive for convex surfaces while negative for concave surfaces in this work.

The transport of this interface with a velocity $\boldsymbol{u}$ is described by the solution of the transport equation given as

$$
\frac{\partial \phi}{\partial t}+\boldsymbol{u} \cdot \nabla \phi=0
$$

In order to mitigate the discontinuities in the solution of this equation and to avoid singularities in the distance function $\phi$, we have implemented a fifth-order WENO scheme for discretizing the convective term in Equation (3). The temporal derivative is discretized using forward Euler scheme. A directionally split advection algorithm [51 is used for solving this transport equation. Due to the very nature of this transport and high velocity gradients, spreading and stretching of the level set function may occur thereby losing its distance function property $\left(\|\boldsymbol{\nabla} \phi\|_{2}=1\right)$. A redistancing algorithm [49] is thus applied to regain this property.

The main drawback of the level set methods is the mass loss especially in the under-resolved regions of the computational domain. One way to mitigate this problem is by coupling level set with the volume of fluid (VOF) method as presented by Sussman and Puckett [17].

\subsubsection{Volume fraction}

The VOF method [5, 52, 11] uses the liquid volume fraction which is the fraction of the liquid in a computational cell. Within this work, the liquid/gas interface is represented in a piecewise linear interface calculation (PLIC) sense with the idea that a planar interface approximates the reference/original interface. The liquid volume fraction $F$ is defined as

$$
F(\boldsymbol{x}, t)=\frac{1}{\left|C_{\Omega}\right|} \int_{C_{\Omega}} H(\phi(\boldsymbol{x}), t) d \boldsymbol{x},
$$

where $C_{\Omega}$ represents a computational cell in the numerical simulation domain $\Omega$ and $H$ is the Heaviside function expressed as

$$
H(\phi(\boldsymbol{x}))=\left\{\begin{array}{l}
1, \text { if } \phi(\boldsymbol{x})>0 \\
0, \text { otherwise }
\end{array}\right.
$$

with the sign convention of the level set function $\phi$ taken as

$$
\phi(\boldsymbol{x})=\left\{\begin{array}{l}
>0, \text { if } \boldsymbol{x} \text { is inside liquid phase } \\
<0, \text { if } \boldsymbol{x} \text { is inside gas phase, }
\end{array}\right.
$$

Thus, $F$ obey the bounds of $0 \leq F \leq 1$. The gas phase volume fraction is given as $1-F$. The physical properties of the phases $\alpha$ in a computational cell, which can be density $\rho$ or viscosity $\mu$, are determined using $F$ as $\alpha(\boldsymbol{x})=\alpha_{\mathrm{liq}} F(\boldsymbol{x})+\alpha_{\text {gas }}(1-F(\boldsymbol{x}))$. This expression involves an assumption that the physical properties are constant within each phase.

The interface described within the context of VOF method is advected according to the following transport equation

$$
\frac{\partial F}{\partial t}+\boldsymbol{u} \cdot \nabla F=0
$$

A directionally-split algorithm proposed by Weymouth and Yue 51] is used for advecting the liquid volume fraction. To be consistent with this algorithm, the following modified form of the transport equation for incompressible divergence free flow is solved

$$
\frac{\partial F}{\partial t}+\nabla \cdot(F \boldsymbol{u})=c(\boldsymbol{\nabla} \cdot \boldsymbol{u}) ; \quad c=\left\{\begin{array}{l}
1, F>0.5 \\
0, \text { otherwise }
\end{array}\right.
$$

In each direction of advection, the liquid volume fraction is advected along one-dimensional velocity which is not divergence-free. Thus, the dilatation term $c(\boldsymbol{\nabla} \cdot \boldsymbol{u})$ appear in the modified form of the equation; otherwise, jetsam and flotsam occurs in the computational domain and the bounds of $F$ will not be respected. 
Due to the directionally-split nature of the transport, the advection directions are swapped between consecutive time steps, i.e.,

$$
\begin{aligned}
t^{n} \rightarrow t^{n+1} & : x-y-z \\
t^{n+1} \rightarrow t^{n+2} & : y-z-x \\
t^{n+2} \rightarrow t^{n+3} & : z-x-y
\end{aligned}
$$

repeat.

It is to be remarked that there is no mass loss observed in the computational domain using this directionallysplit algorithm.

\subsubsection{Coupling level set and VOF methods (CLSVOF method)}

The main idea of coupling level set framework with VOF method is to exploit the advantage of each of the methodologies which is to minimize the loss of mass and to preserve the description as well as geometrical properties of the interface. To that end, the coupling between the two methods are carried out according to the procedure described by Ménard et al. [18] in the context of CLSVOF method. In this method, the unit normal of the liquid/gas interface is computed using the least mean square approach [17. The coupling mainly involves the mutual correction of the level set and the liquid volume fraction values in the computational domain. In order to ensure that an accurate measure of level set and the geometrical properties of the interface are preserved, care is taken in correcting the level set signed distance function based on the liquid volume fraction in each computational cell. The redistancing procedure for the level set function is performed as described by [18 to regain the signed distance function property. Appendix A presents details on the implementation and algorithm of the CLSVOF method employed in this work.

\subsection{Moment of Fluid (MOF) method}

The CLSVOF method has been shown 48, 53, 54 to be successful in simulating the incompressible multiphase flows for various configurations. However, such a state-of-the-art numerical method can still fail in the computation of the geometrical properties for under-resolved interface topologies [18. To test this hypothesis, we consider a $2 \mathrm{D}$ liquid ligament placed in $[0,1] \times[0,1]$ domain. The domain is discretized using a $32^{2}$ mesh resulting in an equidistant mesh spacing of $\Delta x=\Delta y=0.03125$. The thickness of the ligament is specifically chosen to be $1.5 \Delta x$ which qualifies it to be under-resolved (since thickness $<2 \Delta x$ ). This is because at least two computational cells are needed for an interface to be resolved typically for an interface reconstruction method. The test comprises of advection of this ligament along the $x$-direction (horizontal direction) of the doubly periodic domain with a velocity of $u=1.0, v=0.0$, and $\mathrm{CFL}=0.5$ with predictorcorrector time integration scheme using CLSVOF method [18] in the ARCHER solver. The Navier-Stokes equations are not solved in this test but only the phase interface transport equations pertaining to liquid volume fraction (i.e., Equation (7)) and level set (i.e., Equation (3)) are solved. The conclusion of the test is determined at the time instant $t=T=1.0$ at which the ligament comes back to its initial location. The results from this test shown in Figure 1 demonstrates that the shape of the ligament is deformed at the end of the advection process. This highlights the limitation of the interface reconstruction method in capturing the under-resolved liquid structure that is physically relevant. Such under-resolved liquid ligaments are commonly observed in a large scale simulations of liquid atomization.

One of the ways to accurately capture the under-resolved liquid structures [55] and preventing artificial breakup has been demonstrated using the moment of fluid (MOF) method 22]. In one of our previous works 45], we have shown the higher accuracy of MOF method for simulating the incompressible multiphase flows. When the MOF method is used for the same test of under-resolved ligament advection under the same test conditions and using numerical schemes, we get the result as shown in Figure 2. Upon comparing Figures 1 and 2, we find that there are no corrugations or change of shape of the under-resolved liquid ligament when using the MOF method. This goes to show that MOF method is able to preserve the interface orientation and shape of the liquid structure during advection. 


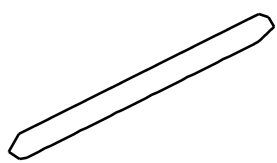

(a) $t=0$

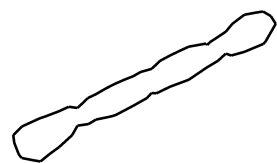

(b) $t=T / 2$

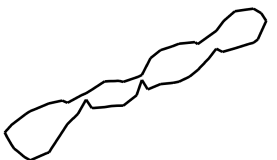

(c) $t=T$

Figure 1: Phase interface shape of liquid ligament obtained using CLSVOF method of interface reconstruction. The velocity field employed for advecting the ligament is $u=1, v=0, w=0$ with the advection direction from left to right in the computational domain.

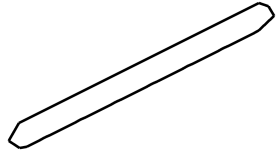

(a) $t=0$

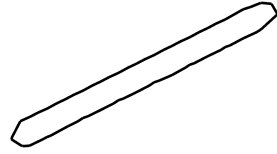

(b) $t=T / 2$

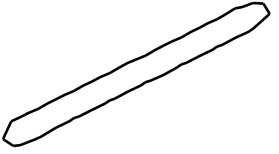

(c) $t=T$

Figure 2: Phase interface shape of liquid ligament obtained using MOF method of interface reconstruction. The velocity field employed for advecting the ligament is $u=1, v=0, w=0$ with the advection direction from left to right in the computational domain.

\subsubsection{Interface reconstruction}

The MOF method is to be considered as a superset of the classical VOF method as it uses both liquid phase volume fraction (zeroth moment of liquid volume) as well as centroids/center of mass (COM) of liquid and gas phase (first moment of liquid volume) in each computational cell to reconstruct the interface. Within the context of the MOF method, the liquid volume fraction (defined in Equation (4)) and the liquid phase centroid are computed as

$$
\begin{aligned}
F & =\frac{\int_{\omega} d \boldsymbol{x}}{\int_{\Omega} d \boldsymbol{x}}, \\
\boldsymbol{x}_{\mathrm{COM}} & =\frac{\int_{\omega} \boldsymbol{x} d \boldsymbol{x}}{\int_{\Omega} d \boldsymbol{x}},
\end{aligned}
$$

while the gas phase volume fraction and its corresponding phase centroid or center of mass (COM) are defined as

$$
\begin{aligned}
F^{\mathrm{gas}} & =\frac{\int_{\omega / \Omega} d \boldsymbol{x}}{\int_{\Omega} d \boldsymbol{x}}, \\
\boldsymbol{x}_{\mathrm{COM}}^{\mathrm{gas}} & =\frac{\int_{\omega / \Omega} \boldsymbol{x} d \boldsymbol{x}}{\int_{\Omega} d \boldsymbol{x}},
\end{aligned}
$$

where $\boldsymbol{x}_{\mathrm{COM}}$ is the phase centroid, and $\omega$ is the domain of the liquid packet (with its volume denoted by $|\omega|$ ) inside the computational cell $C_{\Omega}$ (with its volume denoted by $\left|C_{\Omega}\right|$ ) and $\omega / \Omega$ represents the region within the computational cell outside the liquid phase (i.e., gas phase region).

The MOF method [45] employed in this work was developed in a PLIC sense to approximate the reference/original interface. Thus, the equation of the reconstructed interface plane in 3D (line in 2D) is given 
as $a x+b y+c z+d=0$ where interface unit normal $\boldsymbol{n}=[a, b, c]^{T}$. The interface reconstruction involves determining the components of unit normal $\boldsymbol{n}$ and the distance $d$ which is the shortest distance of the computational cell center to the interface. This is carried out as the solution to a constrained optimization problem wherein both $\boldsymbol{n}$ and $d$ have to be simultaneously determined such that volume is conserved (Equation (14)) and centroid defect $E^{\mathrm{MOF}}$ is minimized (Equation 15 )

$$
\begin{aligned}
& \left|F^{\mathrm{ref}}-F^{\mathrm{act}}(\boldsymbol{n}, d)\right|=0, \text { and } \\
& E^{\mathrm{MOF}}(\boldsymbol{n}, d)=\left\|\boldsymbol{x}_{\mathrm{COM}}^{\mathrm{ref}}-\boldsymbol{x}_{\mathrm{COM}}^{\text {act }}(\boldsymbol{n}, d)\right\|_{2} .
\end{aligned}
$$

The centroid defect $E^{\mathrm{MOF}}$ is the distance between the phase centroids of the reference and reconstructed interfaces. It is to be remarked that all the variables with the superscript "ref" pertain to the reference (or original) interface while those with "act" pertain to the actual (or reconstructed) interface. For the purpose of illustration, Figure 3 shows a typical 3D computational cell with an interface and its $2 \mathrm{D}$ front view with the reference interface (solid curved line) and PLIC reconstructed interface (dashed straight line) based on liquid as reference fluid.

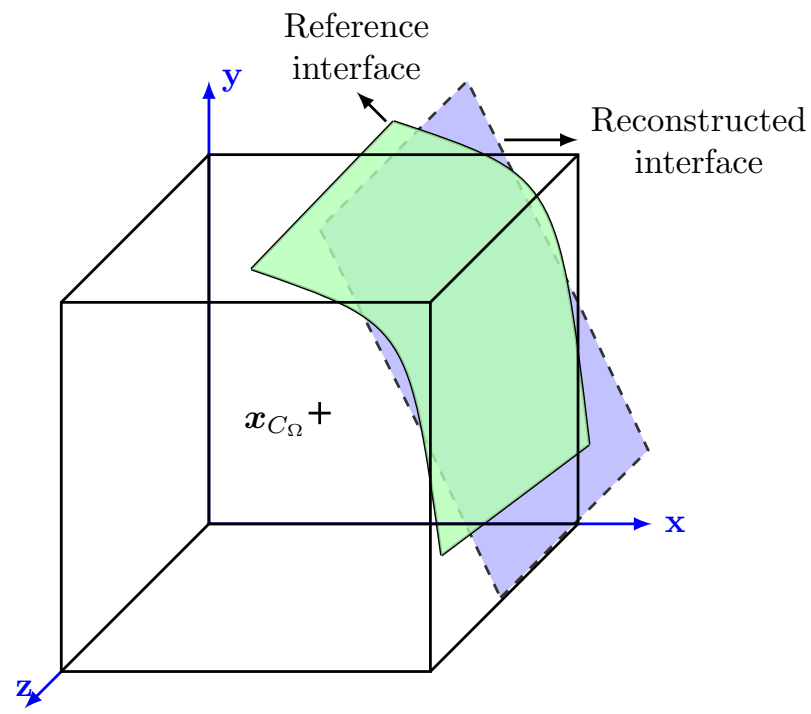

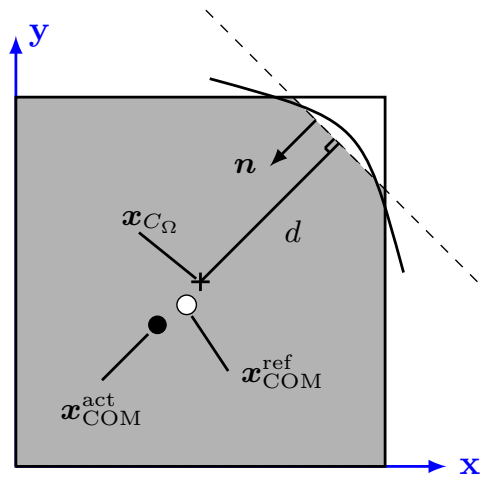

(b) Front view with centroids.

(a) 3D hexahedral computational cell.

Figure 3: Exemplary computational cell in 3D (in (a)) with its front view (in (b)) labelled with reference interface (curved solid line), reconstructed interface (straight dashed line), interface unit normal, and liquid phase centroids of reference interface (solid circle) and reconstructed interface (hollow circle), liquid phase region (gray region).

The MOF method [45, in our in-house solver ARCHER, reconstructs the liquid/gas interface in the following manner based on the methodology of Jemison et al. [27. First, the reference phase (between liquid and gas phases) is chosen such that its centroid is farthest from the cell center. Second, an initial guess for the components of unit normal (i.e., $\boldsymbol{n}^{0}=\left[a^{0}, b^{0}, c^{0}\right]^{T}$ ) are computed from the gradient of the local value of the level set function, i.e.,

$$
a^{0}=\frac{\phi_{i+1, j, k}-\phi_{i-1, j, k}}{2 \Delta x}, \quad b^{0}=\frac{\phi_{i, j+1, k}-\phi_{i, j-1, k}}{2 \Delta y}, \quad c^{0}=\frac{\phi_{i, j, k+1}-\phi_{i, j, k-1}}{2 \Delta z}
$$

Using this initial guess, the value of $d$ is determined by solving Equation (14) using a geometric method [56] upto the machine precision. Then, in order to compute the interface unit normal, it is expressed the interface unit normal in polar coordinates as

$$
\boldsymbol{n}=\left[\begin{array}{l}
a \\
b \\
c
\end{array}\right]=\left[\begin{array}{c}
\sin \Phi \cos \Theta \\
\sin \Phi \sin \Theta \\
\cos \Phi
\end{array}\right] .
$$


using which $\Phi^{0}$ and $\Theta^{0}$ are computed. Next, using the values of $\Phi^{0}, \Theta^{0}$, and $d$, the (chosen) actual (i.e., reconstructed) phase centroid $\boldsymbol{x}_{\mathrm{COM}}^{\text {act }}$ is computed. It is to be remarked that coordinates of the $\boldsymbol{x}_{\mathrm{COM}}^{\mathrm{ref}}$ are already available based on the liquid volume fraction data (c.f. Section 2.2.2). Once the coordinates of the actual centroid are determined, the Gauss-Newton iterative algorithm is used to minimize the centroid defect $E^{\mathrm{MOF}}$ yielding the optimal value of $\Phi$ and $\Theta$ (and by extension optimal $\boldsymbol{n}$ ). The procedure to determine these optimal values is given in Algorithm 1 where $\boldsymbol{g}(\Phi, \Theta, d)=\boldsymbol{x}_{\mathrm{COM}}^{\mathrm{ref}}-\boldsymbol{x}_{\mathrm{COM}}^{\text {act }}(\Phi, \Theta, d)$ is the objective function (representing $E^{\mathrm{MOF}}$ ) to be minimized, $\boldsymbol{J}$ is the Jacobian matrix of this objective function, ctr is the iteration counter number, tolg and tolJ are the tolerance values. For more details on the implementation of the MOF method and the algorithms on minimization of the centroid defect, the reader is referred to our previous work [45].

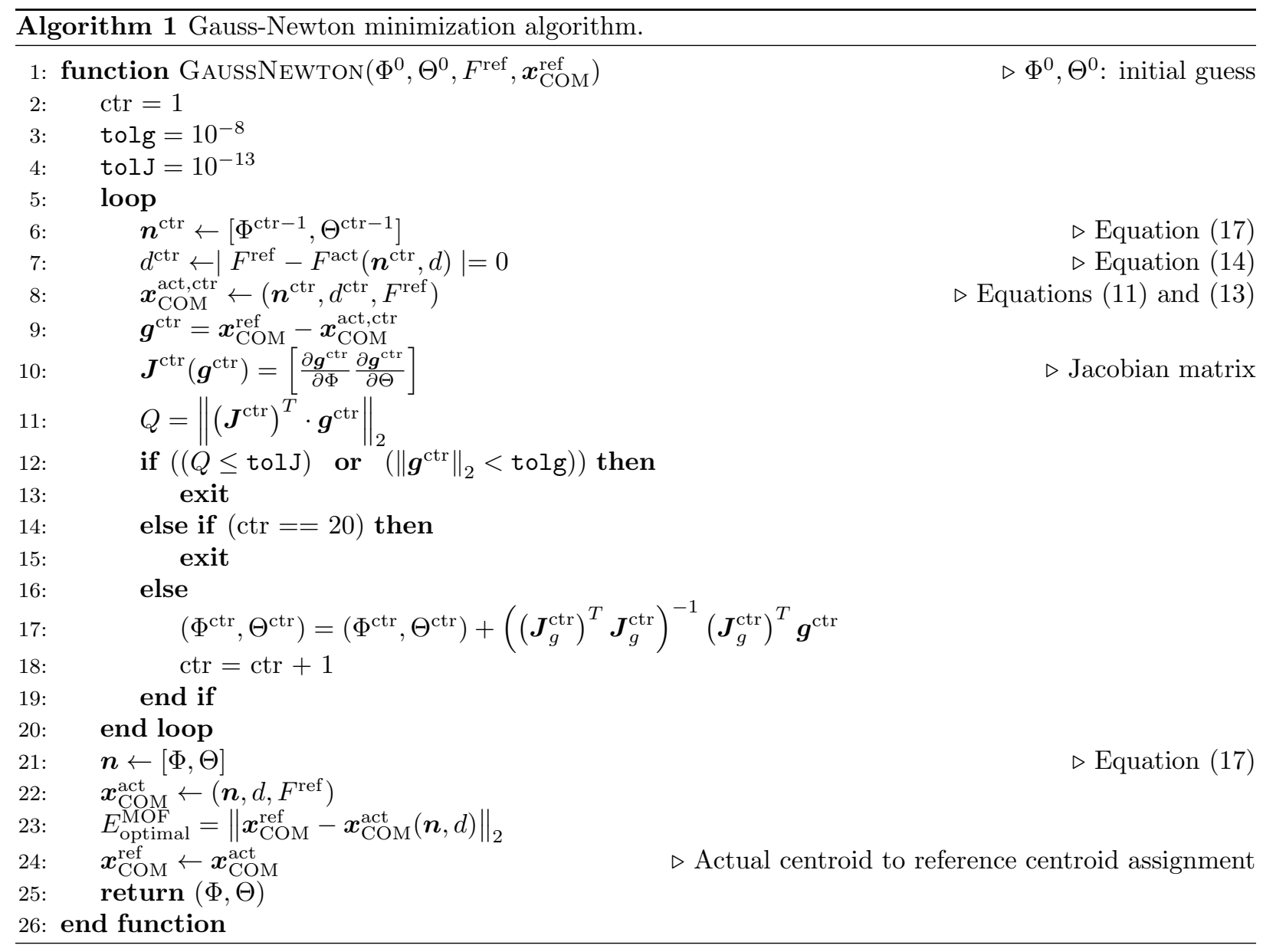

\subsubsection{Computation of reference centroid}

The methodology involved in the computation of the phase centroid is explained as follows. It is to be remarked that the interface reconstruction is needed only in the mixed cells (i.e., cells with both liquid and gas phases), thus, the centroid computation is performed only for such cells. For the cells with only liquid phase (full cell) or gas phase (empty cell), the location of the phase centroid coincide with that of the computational cell center. In fact, it is sufficient to know the coordinates of the centroid of one phase to compute those for the other phase since the phase centroids are linked to each other in each computational cell according to the following relation,

$$
V_{\text {liq }} x_{\mathrm{COM}}^{\text {liq }}+V_{\text {gas }} \boldsymbol{x}_{\mathrm{COM}}^{\mathrm{gas}}=V_{\text {cell }} \boldsymbol{x}_{C_{\Omega}},
$$


where $V_{\text {liq }}$ is the volume of liquid, $V_{\text {gas }}$ is the volume of gas, $\boldsymbol{x}_{C_{\Omega}}$ is the coordinates of the cell center, and $V_{\text {cell }}$ is the volume of the computational cell, i.e., $V_{\text {cell }}=\Delta x \times \Delta y \times \Delta z$.

We now consider an exemplary hexahedral computational cell with the liquid/gas interface shown in Figure 4a. The liquid phase under the interface is shown in gray color in Figure $4 \mathrm{~b}$ and the unit normal of the interface points towards the liquid phase. The basic idea of the phase centroid computation revolves

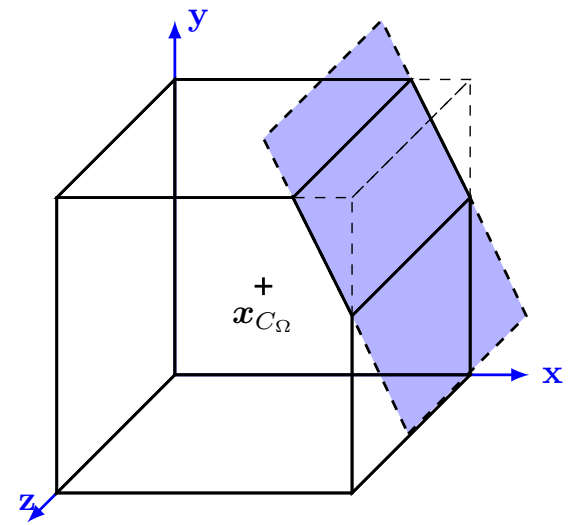

(a) 3D hexahedral computational cell.

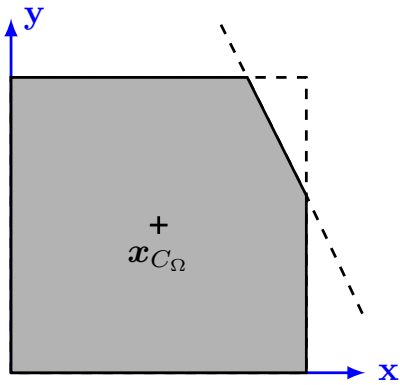

(b) Front view with liquid phase shown in gray.

Figure 4: Exemplary computational cell used in ARCHER flow solver mesh.

around the triangulation of the corresponding phase in the cell. The premise of this algorithm pivots to the equispaced hexahedral cells (i.e., $\Delta x=\Delta y=\Delta z$ ) in the mesh for discretizing the domain. Thus, the total number of vertices in each cell is 8 and total number of faces in a cell is 6 remaining constant. For the sake of simplicity and without loss of generality, the steps described below pertain to the computation of coordinates of the centroid of the liquid phase for the hexahedral cell shown in Figure 4a. The algorithm for the computation of centroid of the gas phase can be derived in a straightforward manner based on the below procedure. The following steps are implemented to compute the coordinates of the phase centroid.

(a) First, the computational cell is transformed from its global coordinates (i.e., $\left.\left(x_{1}, y_{1}, z_{1}\right) \ldots\left(x_{2}, y_{2}, z_{2}\right)\right)$ to local coordinates $((0,0,0) \ldots(1,1,1))$ (c.f. Figures $5 \mathrm{a}$ and $5 \mathrm{~b}$ ).

(b) Then, the coordinates of the points of intersection of the interface with the cell faces are computed. The cell formed by the intersection of the original hexahedral computational cell and the interface will be called as truncated cell (shown in Figure 5c).

(c) Using the vertices of this truncated cell, the barycenter of the liquid phase is computed within the local coordinate system of the computational cell (shown in Figure 5d).

(d) Then, each face of this truncated cell is triangulated to subsequently form tetrahedral elements (see Figure 5 e.

(e) Using the liquid phase barycenter as apex of each tetrahedron, triangulation is performed to form tetrahedra as shown in Figure $5 f$.

(f) The volume $V_{\text {tetra }}$ and barycenter $\boldsymbol{x}_{\text {tetra }}$ of each tetrahedron are then computed. The expression for the volume of tetrahedron with vertices $\boldsymbol{a}=\left(a_{1}, a_{2}, a_{3}\right), \boldsymbol{b}=\left(b_{1}, b_{2}, b_{3}\right), \boldsymbol{c}=\left(c_{1}, c_{2}, c_{3}\right)$, and $\boldsymbol{d}=\left(d_{1}, d_{2}, d_{3}\right)$ is given as

$$
V_{\text {tetra }}=\frac{1}{6}|\operatorname{det}(\boldsymbol{a}-\boldsymbol{d}, \boldsymbol{b}-\boldsymbol{d}, \boldsymbol{c}-\boldsymbol{d})|
$$


The barycenter of the tetrahedron $\left(x_{\text {tetra }}, y_{\text {tetra }}, z_{\text {tetra }}\right)$ is computed as

$$
\begin{aligned}
x_{\text {tetra }} & =\frac{1}{4}\left(a_{1}+b_{1}+c_{1}+d_{1}\right) \\
y_{\text {tetra }} & =\frac{1}{4}\left(a_{2}+b_{2}+c_{2}+d_{2}\right) \\
z_{\text {tetra }} & =\frac{1}{4}\left(a_{3}+b_{3}+c_{3}+d_{3}\right)
\end{aligned}
$$

It is to be noted that the barycenter and the centroid of tetrahedron coincide. Hence, for each tetrahedron, the barycenter will be hereon referred as centroid.

(g) The centroid of liquid phase in this 3D hexahedral computational cell is then computed as the volume weighted average of the centroids of each tetrahedron weighted using the tetrahedral volume. This is expressed mathematically as

$$
\boldsymbol{x}_{\mathrm{COM}}=\frac{\sum_{i_{\text {tetra }}=1}^{N_{\text {tetra }}} V_{i_{\text {tetra }}} \boldsymbol{x}_{i_{\text {tetra }}}}{\sum_{i_{\text {tetra }}=1}^{N_{\text {tetra }}} V_{i_{\text {tetra }}}}
$$

The steps described are pictorially illustrated in Figure 5. The overall algorithm for this methodology is given in Algorithm 2

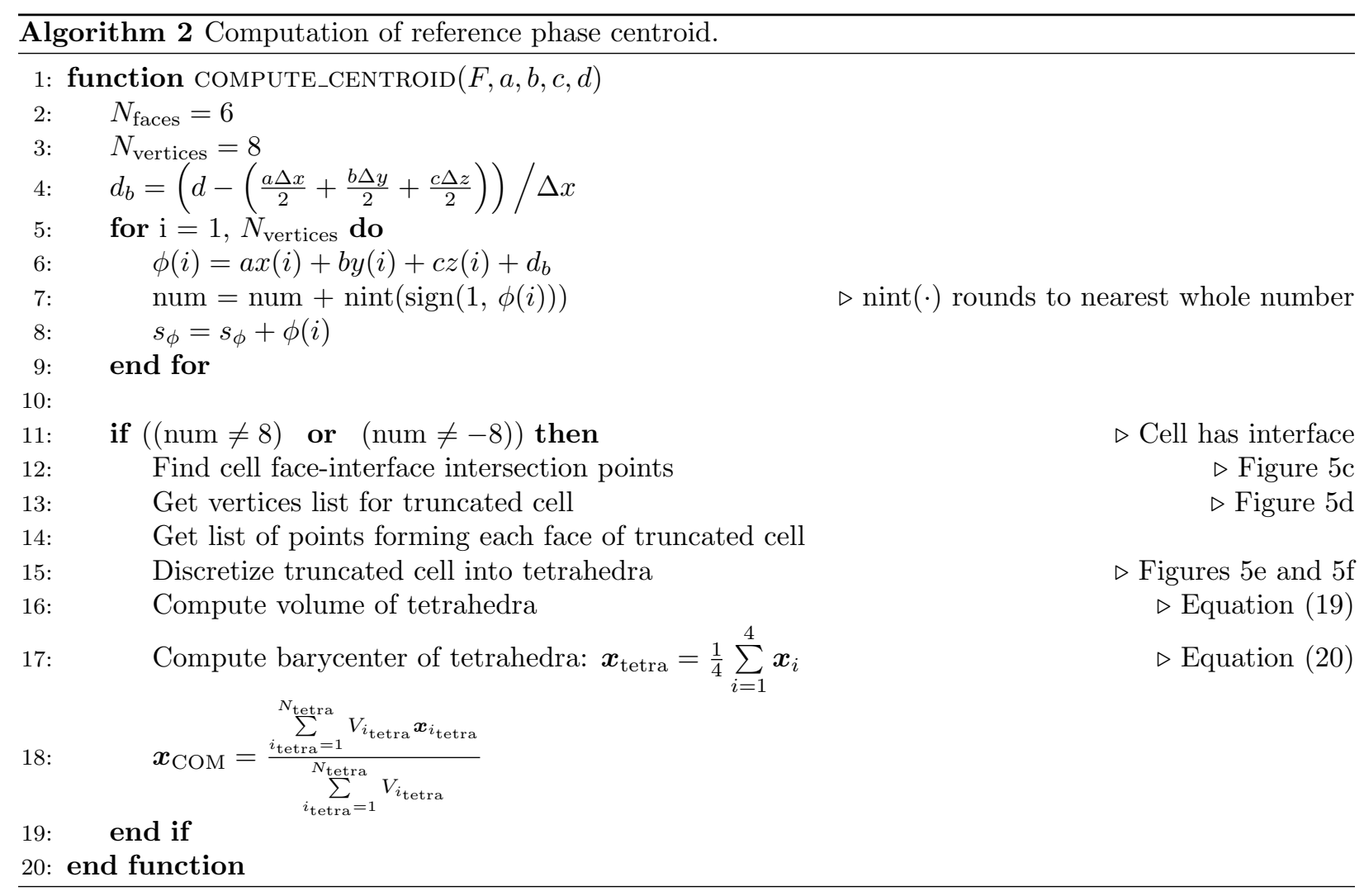

\subsubsection{Phase transport}

The transport of the liquid phase (and gas phase) within the context of MOF method involves the transport of the liquid volume fraction $F$ and the reference phase centroids $\boldsymbol{x}_{\mathrm{COM}}^{\mathrm{ref}}$. The transport of $F$ (c.f. 


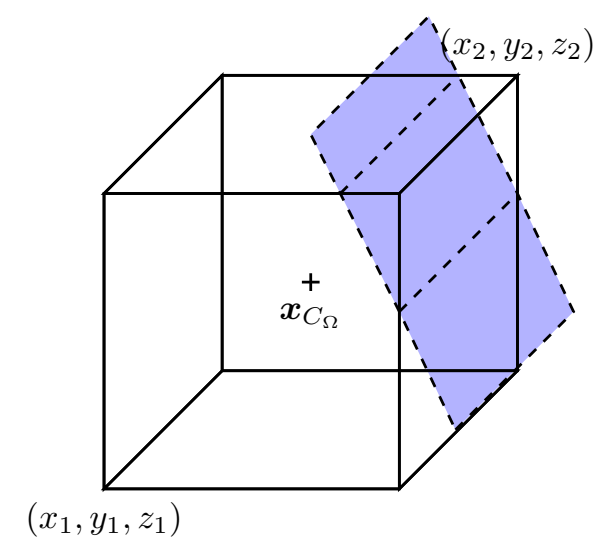

(a) Cell in global coordinates.

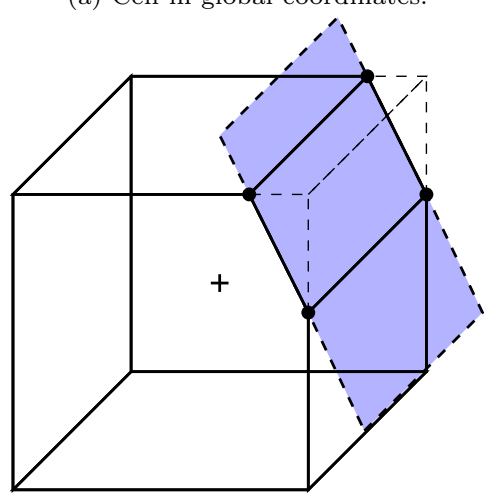

(c) Cell face-interface intersection points.

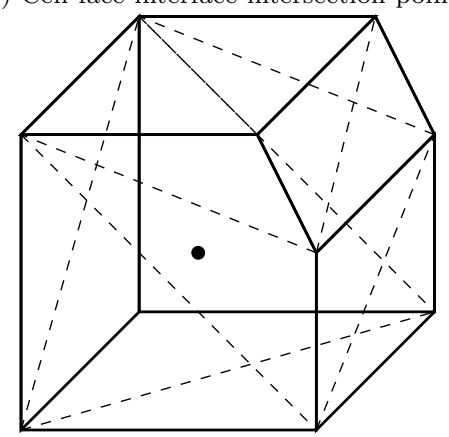

(e) Formation of diagonals on cell faces.

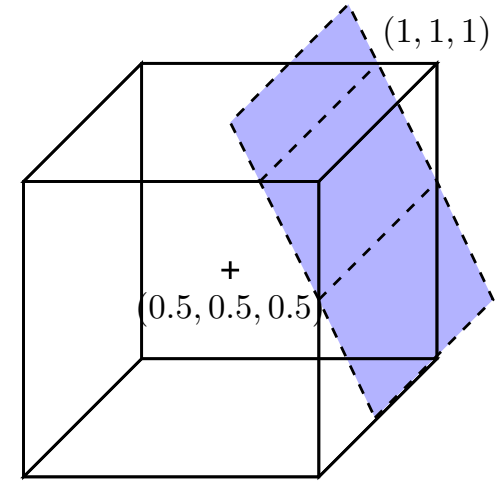

$(0,0,0)$

(b) Cell in local coordinates.

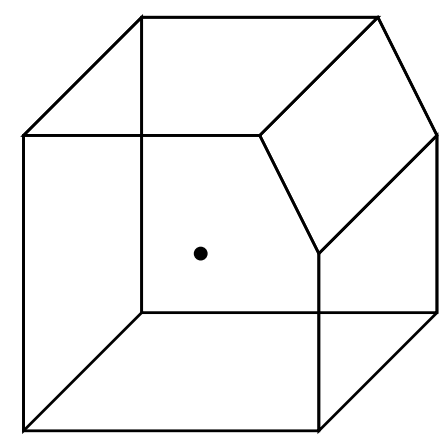

(d) Liquid phase barycenter and truncated cell.

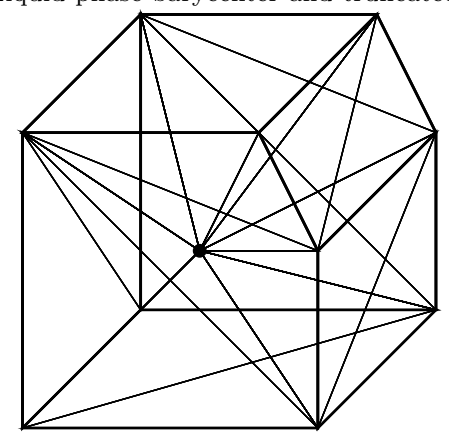

(f) Tetrahedral elements.

Figure 5: Step-by-step (from (a) to (f)) pictorial representation of liquid phase centroid computation.

Equation (7) ) along with the numerical methods for discretization of the various terms have been shown in Section 2.1.2 The transport of the phase centroid is carried out as an approximated Lagrangian particle associated with its corresponding volume packet (also referred as flux volume) consistent to the literature 22]. The centroid is transported according to the following equation

$$
\frac{\partial \boldsymbol{x}_{\mathrm{COM}}}{\partial t}=\boldsymbol{u}\left(\boldsymbol{x}_{\mathrm{COM}}\right)
$$

where $\boldsymbol{u}\left(\boldsymbol{x}_{\mathrm{COM}}\right)$ is the velocity at the location of the centroid $\boldsymbol{x}_{\mathrm{COM}}^{\text {ref }}$ linearly interpolated from the cell face-centered velocity (see Figure B.43 in Appendix B). The reader is referred to Appendix A of Ref. 22] for the detailed derivation of the Equation (22). It is to be remarked that centroids of liquid and gas phase 
are stored for a mapped unit computational cell, hence, their value is always in the range [0,1]. During the advection step, each phase centroid coordinate is remapped back to the physical computational cell 45. This transport equation is solved using a directionally-split advection algorithm with an Eulerian Implicit-Lagrangian Explicit (EI-LE) scheme. The first order time integration of Equation 22 gives

$$
\boldsymbol{x}_{\mathrm{COM}}^{n+1}=\boldsymbol{x}_{\mathrm{COM}}^{n}+\boldsymbol{u}\left(\boldsymbol{x}_{\mathrm{COM}}^{*}\right) \Delta t .
$$

In the case of Eulerian Implicit (EI) scheme with $\boldsymbol{x}_{\mathrm{COM}}^{*}=\boldsymbol{x}_{\mathrm{COM}}^{n+1}$, the final transport equation derives to be

$$
x_{\mathrm{COM}}^{n+1}=E \times\left(x_{\mathrm{COM}}^{n}-\left(u_{+} x_{-}-u_{-} x_{+}\right)\right)
$$

where $E=1 /\left(1-\left(u_{+}-u_{-}\right)\right), x_{ \pm}=x_{i \pm 1 / 2}$, and $u_{ \pm}=u_{i \pm 1 / 2} \Delta t / \Delta x$ which becomes the local cell-based CFL number and are non-dimensional. On the other hand, for the Lagrangian Explicit (LE) scheme with $\boldsymbol{x}_{\mathrm{COM}}^{*}=\boldsymbol{x}_{\mathrm{COM}}^{n}$, the transport equation derives to be

$$
x_{\mathrm{COM}}^{n+1}=L x_{\mathrm{COM}}^{n}-\left(u_{+} x_{-}-u_{-} x_{+}\right)
$$

with $L=1+\left(u_{+}-u_{-}\right)$. The derivation of Equations (24) and (25) are detailed in Appendix B. The consistency in the advection of phase centroid and liquid volume fraction is achieved by alternating between the EI and LE scheme of advection between consecutive time steps of the simulation, i.e.,

$$
\begin{aligned}
& t^{n} \rightarrow t^{n+1}: x(\mathrm{EI}) \rightarrow y(\mathrm{LE}) \rightarrow z(\mathrm{EI}), \\
& t^{n+1} \rightarrow t^{n+2}: y(\mathrm{EI}) \rightarrow z(\mathrm{LE}) \rightarrow x(\mathrm{LE}), \\
& t^{n+2} \rightarrow t^{n+3}: z(\mathrm{EI}) \rightarrow x(\mathrm{EI}) \rightarrow y(\mathrm{LE}), \\
& \quad \text { repeat. }
\end{aligned}
$$

The centroid transport procedure employed in this work is same as presented by Asuri Mukundan et al. 45 following the work of Jemison et al. [27. Without loss of generality, the procedure for the transport of the reference liquid phase centroid $\boldsymbol{x}_{\mathrm{COM}}^{\text {liq }}$ (the same procedure is employed for gas phase centroid $\boldsymbol{x}_{\mathrm{COM}}^{\text {gas }}$ ) along the $x$-direction ( $y$ - and $z$-direction advection procedure follows the same way) is explained below. We consider the configuration of the three computational cells in $2 \mathrm{D}$ as shown in Figure 6 . In this case,

$$
i-1 \quad i+1
$$

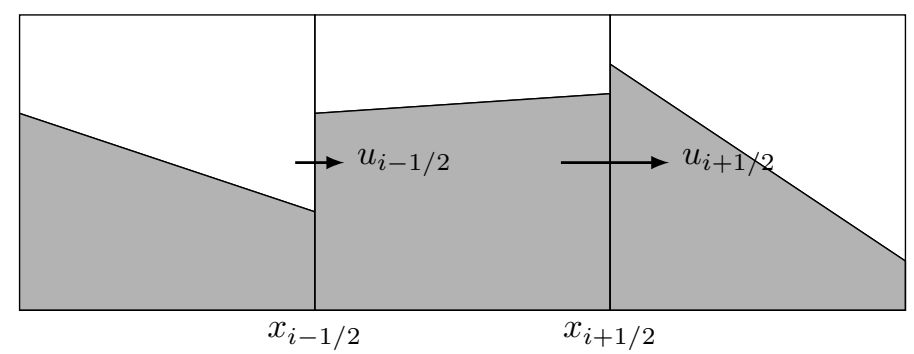

Figure 6: 3-cell stencil for advection of liquid centroid. Liquid depicted as dark fluid.

the liquid from cell $i-1$ moves into cell $i$ and the objective is to find the new centroid of the liquid phase in cell $i$. To that end, the advection procedure is given as follows:

(a) First, we find the region and its amount of liquid volume entering (or displacing) to (or within) cell $i$ hereon called as departure region corresponding to the red dashed outlined region in Figure $7 \mathrm{a}$. Thus,

$$
C_{\Omega_{i_{\text {depart }}}}=\left[x_{i-1 / 2}-u_{i-1 / 2} \Delta t, x_{i+1 / 2}-u_{i+1 / 2} \Delta t\right] \times\left[y_{j-1 / 2}, y_{j+1 / 2}\right] .
$$




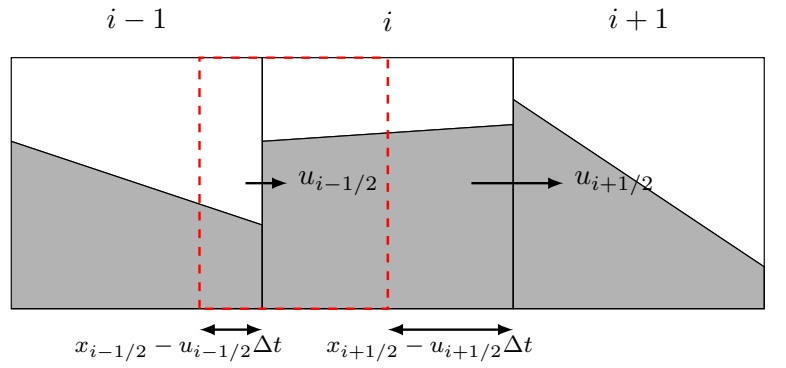

(a) Step 1: Departure region.

$\begin{array}{lll}i-1 & i & i+1\end{array}$

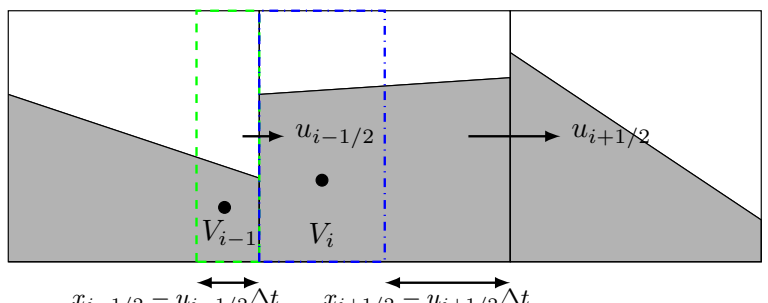

(c) Step 3: Compute phase centroid.

$$
i-1
$$

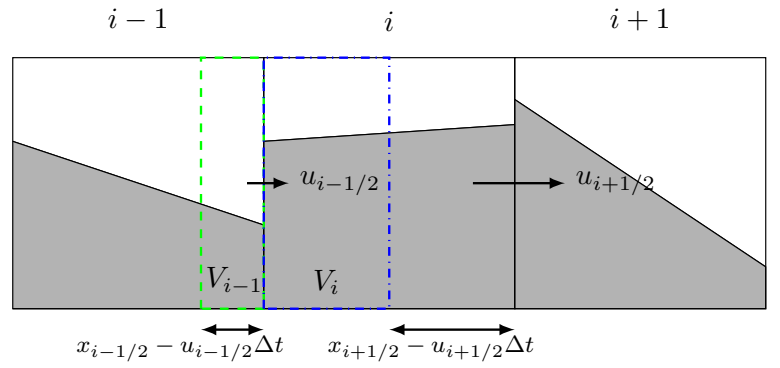

(b) Step 2: Individual departure regions. $i-1$

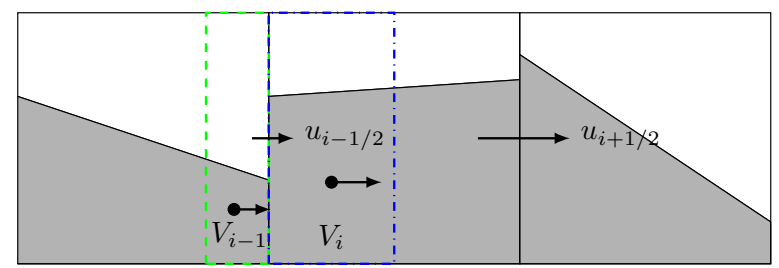

$x_{i-1 / 2}-\underset{i-1 / 2}{\longleftrightarrow} \Delta t \quad x_{i+1 / 2} \stackrel{\longleftarrow u_{i+1 / 2}}{\rightleftarrows} t$

(d) Step 4: Advect phase centroids. $i+1$

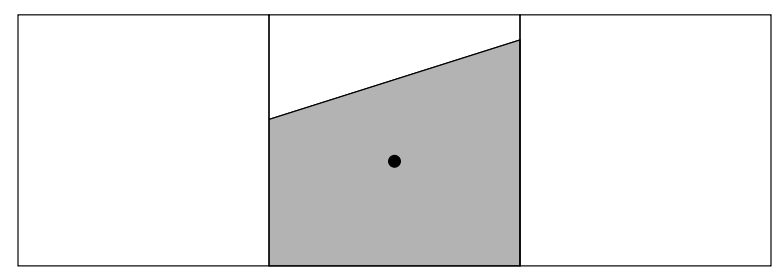

(e) Step 5: Weighted averaged centroid.

Figure 7: Step-by-step (from (a) to (e)) procedure of advection of liquid phase centroid.

(b) Next, we find the domain and volume of individual regions of liquid entering (or displacing) each from cell $i-1$ (dashed outlined region) and $i$ (dashdotted outlined region) hereon called as intersected departure regions as shown in Figure $7 \mathrm{~b}$. Therefore, we have

$$
C_{\Omega_{i^{\prime}, i}}=C_{\Omega_{i+i^{\prime}}} \cap C_{\Omega_{i_{\text {depart }}}} \forall i^{\prime}=-1,0,1 .
$$

The volume of the intersected departure region from cell $i-1$ is denoted by $V_{i-1}$ and the from cell $i$ is denoted by $V_{i}$ in Figure $7 \mathrm{~b}$.

(c) Then, we compute the liquid phase centroid of each of these intersected departure region (c.f. Figure 7c) using Equation (11).

(d) We then advect these centroids using EI-LE scheme according to Equation 22 (c.f. Figure 7d).

(e) Finally, we compute the new liquid phase centroid for cell $i$ as weighted average of all the centroids of liquid phase packets entered (or displaced) within cell $i$ with volume of each liquid packet given as

$$
\boldsymbol{x}_{\mathrm{COM}_{i}}^{n+1}=\frac{\sum_{i^{\prime}=-1}^{1} \boldsymbol{x}_{\mathrm{COM}_{\Omega_{i, i^{\prime}}}}\left|C_{\Omega_{i^{\prime}, i}}\right|}{\sum_{i^{\prime}=-1}^{1}\left|C_{\Omega_{i^{\prime}, i}}\right|},
$$

where $\left|C_{\Omega_{i^{\prime}, i}}\right|$ represent the liquid volume of the corresponding intersected departure region. 
It is to be remarked that the same procedure is employed for the advection of the gas phase centroid. The reader is referred to the work of Asuri Mukundan et al. [45] for detailed explanations on the methodologies, algorithms, and implementation of the MOF method.

\subsection{HyMOFLS: coupling MOF and CLSVOF methods}

In this work, we have developed a coupling between MOF and CLSVOF methods. Such a hybrid method uses MOF method of liquid/gas interface reconstruction for under-resolved regions of the flow while CLSVOF method for the resolved regions of the flow. One unique property of MOF method is its usage of the centroid which CLSVOF does not. Thus, an obvious question is - how are the centroid computation and transport handled in the hybrid method? To that end, we have adopted a strategy in which the reference centroids are computed on-the-fly only for those cells tagged with MOF interface reconstruction. Moreover, the centroid transport is performed only for those cells that are tagged with MOF interface reconstruction method with the anticipation that this cell will again be tagged with MOF method in the next time step. The coupling between the MOF and CLSVOF methods of interface reconstruction is achieved through the local interface curvature computed as

$$
\kappa(\phi)=-\nabla \cdot\left(\frac{\nabla \phi}{\|\nabla \phi\|_{2}}\right),
$$

where $\phi$ represents the level set signed distance function. The curvature is computed using finite difference method with a nine-point stencil in two dimension, the details of which are explained in Ref. [57. The rationale in developing HyMOFLS method is that the MOF method is used for capturing the under-resolved liquid structures (URLS) while the CLSVOF method is used for capturing resolved liquid structures (RLS). Within this work, a liquid structure is defined as under-resolved when it is of the size of the computational mesh spacing. The resolution of the liquid structures is determined according to interface resolution quality (IRQ) 58 for each computational cell that belongs to a liquid structure. The IRQ is expressed as,

$$
\operatorname{IRQ}=\frac{1}{\kappa \Delta x}
$$

where $\Delta x$ is the grid spacing in the computational mesh used for the simulations. The curvature $\kappa$ computed in the cell center in our flow solver is associated to the surface of the liquid/gas interface (if one such exist in the cell). It is to be remarked that the curvature used in Equation (31) is the sum of two principal curvatures of the liquid structures, i.e., $\kappa=\left|\kappa_{1}+\kappa_{2}\right|$. Such a computation of curvature is inspired from the work of Canu [58; however, the drawback with this method of computation is that the curvature becomes infinity on a saddle point of the interfacial surface.

In the past, an IRQ based criterion for differentiating the resolved and under-resolved droplets was used by Wardle and Weller [59] wherein the gradient of liquid volume fraction was used rather than curvature since diffused interface tracking method was employed in their study. An IRQ based differentiation between resolved and under-resolved liquid structures was also used in the work of Anez et al. [1] which used diffused interface tracking methodology. In contrast, our work is focused for the sharp interface capturing method for which interface curvature is a relevant quantity for classification of the resolution of the liquid structures. Moreover, the wrinkling in the interface can be well represented through the local variations in the interface curvature. Hence, the under-resolved liquid structure/resolved liquid structure (URLS/RLS) criterion implemented in this work is

$$
\mathrm{IRQ}=\frac{1}{\kappa \Delta x}=\left\{\begin{array}{l}
<\alpha, \Rightarrow \text { Under-resolved structure } \\
\geq \alpha, \Rightarrow \text { Resolved liquid structure. }
\end{array}\right.
$$

The value of $\alpha=2$ is chosen in this work based on the study by Canu [58; higher value of $\alpha$ tends towards using MOF method of interface reconstruction everywhere in the computational domain. This value necessitates that a minimum of 8 computational cells spanning the length of the major axis of the liquid structure to be classified as RLS else it is URLS. The reader is referred to Appendix C for the derivation of this criterion (Equation (32) and to Section 2.5 for the rationale behind choosing $\alpha=2$. 
In order to find the effect of $\alpha$ on the interface reconstruction, a parametric study for various values of $\alpha$ is performed and presented in Section 2.5. A similar formulation of the IRQ has also been employed by Jemison et al. 27] with the condition that MOF method would be used for interface reconstruction when there are less than 24 cells spanning the length of the major axis of the liquid structure. For more details on the numerical aspect of the URLS/RLS crtierion, the reader is referred to Canu 58 .

The HyMOFLS coupling works as follows. First, we compute the IRQ (Equation (31)) for each computational cell containing the interface using the local mesh spacing and local interface curvature. Then, the URLS/RLS criterion (Equation (32)) is checked in each such cell whose result determines whether the cell belongs to an URLS or a RLS. When the cell belongs to URLS, then MOF method of interface reconstruction is labeled/tagged and used in this cell, else, CLSVOF method is labeled/tagged and used in this cell. The algorithm of cell-labelling with MOF and CLSVOF method based on the URLS/RLS criterion is given in Algorithm 3 . In this algorithm, the variables imin, imax, jmin, jmax, kmin, and kmax correspond to the bounds on the computational cell numbers along the $x-, y-$, and $z$-directions. Moreover, once a cell is identified to be under-resolved (or resolved) the MOF (or CLSVOF) method is labeled to its 26-cell neighbors in 3D (8-cell neighbor in 2D). Such a neighboring cell labelling is performed to avoid local inaccuracies/changes in the interface orientations.

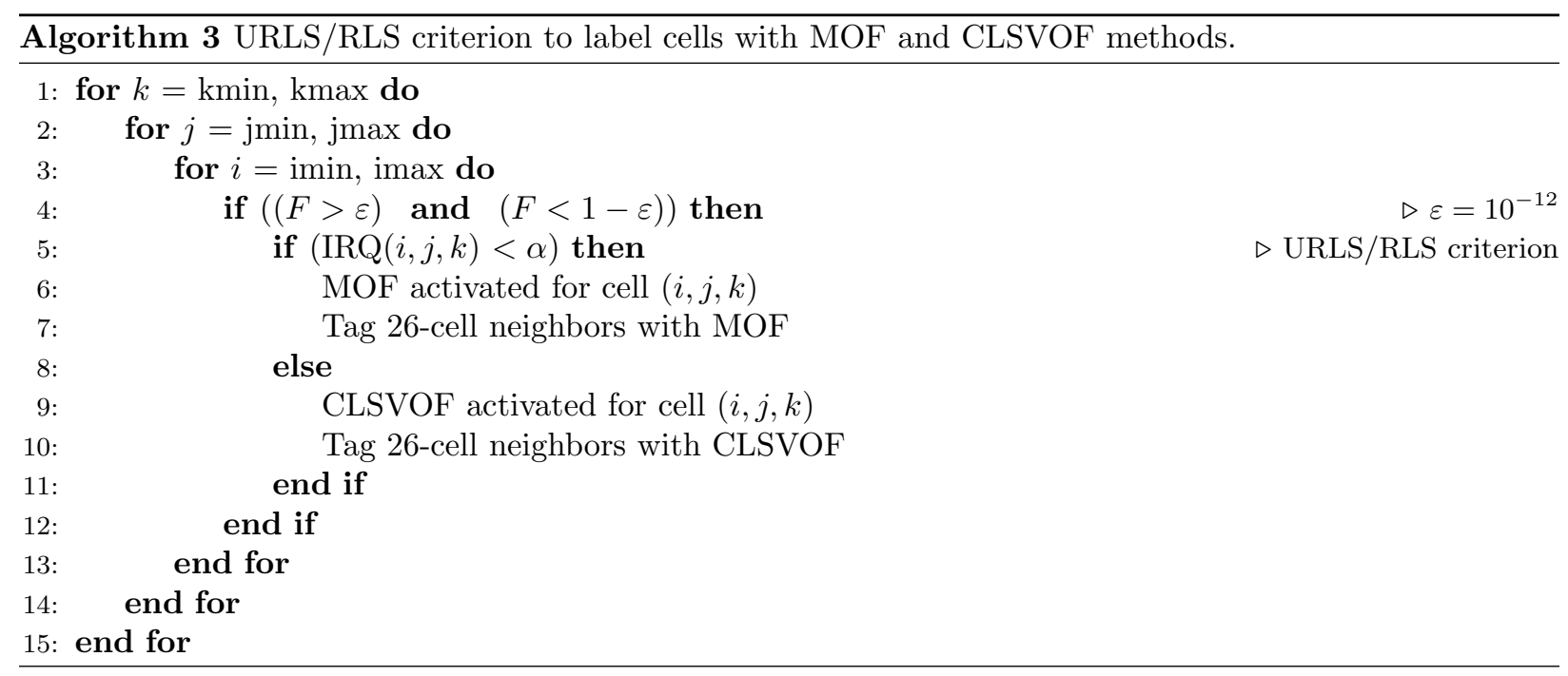

The full algorithm of the implementation of the computation of IRQ value, URLS/RLS criterion (Equation (32) along with the 26-cell neighbor labelling is presented in Algorithm 4. This algorithm of URLS/RLS criterion is implemented in the simulations on multiple processors, hence, care has been taken in ensuring the communications of the MOF and CLSVOF label for each cell are not overwritten for the same cell from other processors (see line numbers 14 and 15 in Algorithm 4). An illustration of tagging of cells (and its neighbors) with MOF and CLSVOF methods within the HyMOFLS framework is shown in Figure 8

In a typical multiphase flows simulations, not all computational cells will be occupied by the liquid structures. In fact, these structures span only a small concentrated region of the whole computational domain. Thus, in order to reduce the computational costs, only the cells containing the interface are checked for determining whether MOF or CLSVOF method need to be used for interface reconstruction. To that end, the liquid volume fraction $F$ in each cell is compared with a threshold value of $\varepsilon=10^{-12}$ in this algorithm. Moreover, as seen in Algorithm 4, the steps involved in tagging a cell to MOF/CLSVOF method and computation of phase centroid for these corresponding cells are split over three loops to mitigate the issues with the miscommunication and over-writing of the tagged label in a cell among the processors. Such miscommunications and over-writings often occurs for the computational cells that are on the processor boundaries. 


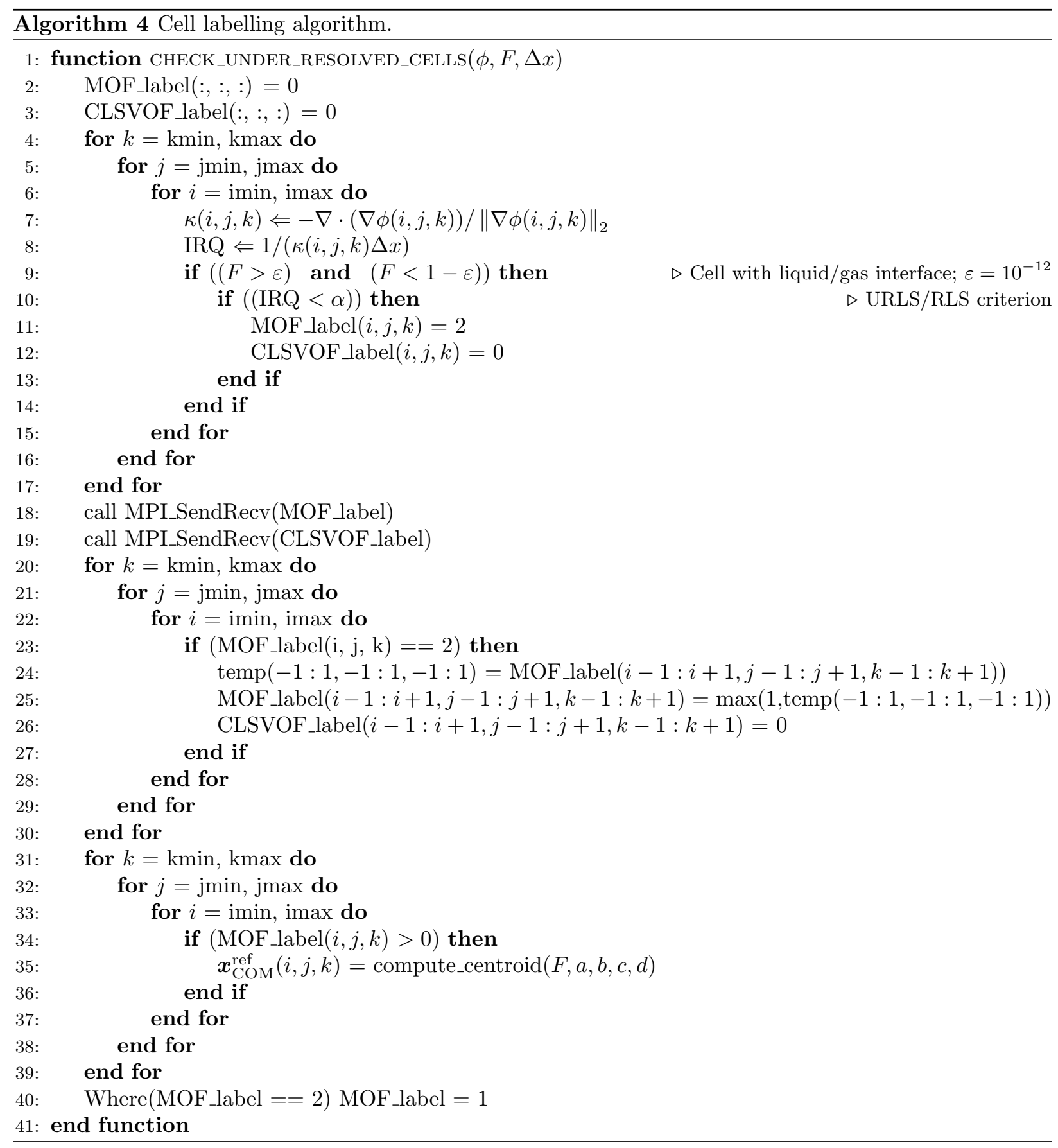

\subsection{Computational comparison tests}

Having described the HyMOFLS method, numerical tests are now performed to find the accuracy of the interface reconstruction and transport. The default value of the parameter $\alpha$ is chosen to be 2 for the tagging of computational cells with MOF or CLSVOF method. Three different tests are presented in this subsection - notched disk rotation, 2D, and 3D droplet deformation. For each test, the results from HyMOFLS, MOF, and CLSVOF interface reconstruction methods such as the shapes of phase interface, error norms (symmetric difference error and geometric error), computational time will be compared. Finally, a parametric study of 


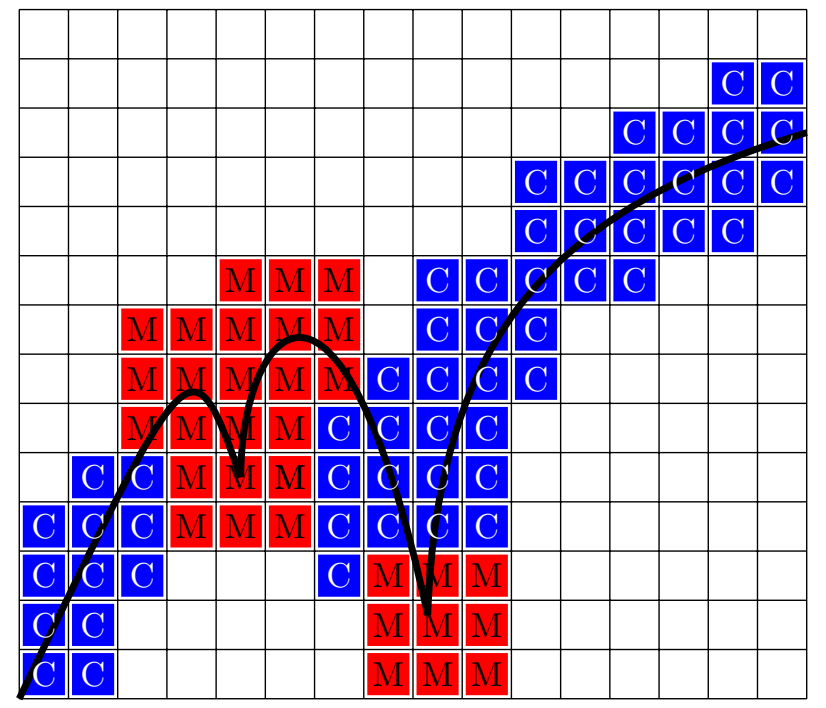

\section{$\mathrm{C}=\mathrm{CLSVOF}$ $\mathrm{M}=\mathrm{MOF}$}

Figure 8: Narrow band of the MOF method tagged cells (red cells with "M" label) and CLSVOF method tagged cells (blue cells with "C" label) around the interface (shown in black solid line).

the threshold value $\alpha$ along with an alternative cell tagging criterion are presented. In each test, the CFL is fixed to a value of 0.5 and the time integration is performed using forward Euler method.

The error norms considered for the quantitative analyses are:

- Symmetric difference error: This error measures the difference in volume in three dimensions (area in two dimensions) between the two interfaces (reference and reconstructed interfaces) giving a measure of the accuracy in the orientation of the interface normal in addition to the amount of liquid volume encompassed under the interface. It is shown as the gray region in Figure 9. The computation of this

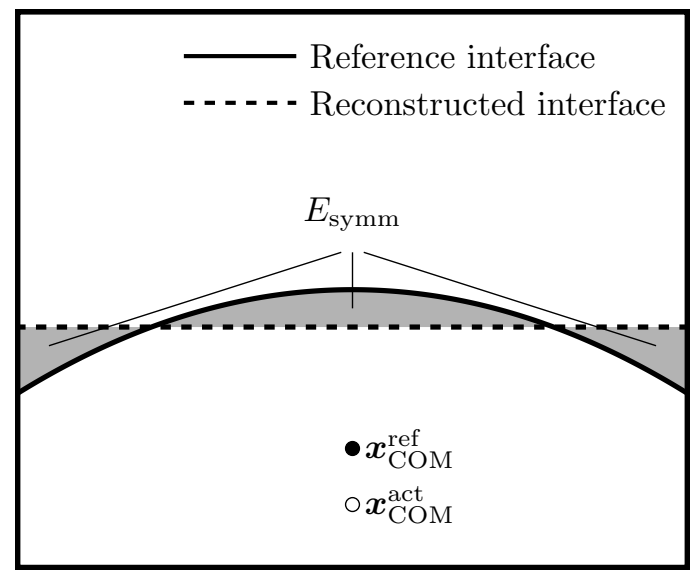

Figure 9: Illustration of symmetric difference area error shown in grey with reference interface (solid line) and reconstructed interface (dashed line).

error can be mathematically expressed as

$$
E_{\text {symm }}=\left|\omega^{\text {ref }} \cup \omega^{\text {act }}-\omega^{\text {ref }} \cap \omega^{\text {act }}\right|
$$


This expression can be simplified in terms of the Heaviside function $H$ as

$$
E_{\text {symm }}=\sum_{i, j, k} \int_{C_{\Omega_{i, j, k}}}\left|H\left(\boldsymbol{n} \cdot\left(\boldsymbol{x}-\boldsymbol{x}_{\boldsymbol{C}_{\boldsymbol{\Omega}}}\right)+d\right)-H\left(\phi_{\text {exact }}(\boldsymbol{x})\right)\right| d \boldsymbol{x},
$$

where $\phi_{\text {exact }}$ is the level set function of the exact interface that determined analytically. The integral in this expression is evaluated using quadrature method by dividing each computational cell $C_{\Omega_{i, j, k}}$ into 128 subcells in each spatial direction.

- Geometric error: this error measures the difference in the shape between reference and reconstructed interfaces. It is computed as

$$
E_{\text {geo }}=\int_{\Omega}|F(\boldsymbol{x}, T)-F(\boldsymbol{x}, 0)| d \boldsymbol{x} .
$$

Both these error norms are computed for all the following time-periodic numerical tests at the end of their time period, i.e., at $t=T$ where $T$ is the time period. The computational time presented in these tests correspond to the average CPU time consumption per timestep which is computed as $t_{\mathrm{CPU}}^{\text {avg }}=t^{\text {total }} \times N_{\text {proc }} / N_{x}$ where $N_{x}$ is the number of computational cells along $x$-direction in the domain.

\subsubsection{Notched disk rotation}

In this test case, a notched Zalesak's disk is revolved around the center of a $[0,1] \times[0,1]$ domain. The disk with a diameter of $D=0.30$ contains a notch with its width 0.06 and length 0.2 with its center located at the $(0.5,0.75)$. The test concludes when the disk comes back to its initial position after one full revolution around the center of the domain based on the following velocity field

$$
\begin{aligned}
u & =\frac{\pi}{3.14}(0.5-y), \quad \text { and } \\
v & =\frac{\pi}{3.14}(x-0.5) .
\end{aligned}
$$

In order to check the order of convergence of the error norms, multiple mesh resolutions ranging from $32^{2}$ to $1024^{2}$ have been used to discretize the domain.

Figure 10 shows the phase interface shape after one full rotation of the notched disk around the domain center for HyMOFLS, MOF, and CLSVOF methods obtained using $64^{2}$ mesh resolution (i.e., $D / \Delta x=$ 19.2). The final interface solution (dashed lines) are compared against the initial (exact) solution (black solid line) for each method of interface reconstruction. By the formulation of the HyMOFLS method, the cells containing the interface are tagged either with MOF or CLSVOF method of interface reconstruction. Consequently, the interface gets the red color when the cell is tagged with MOF method and blue color with CLSVOF method as shown in Figure 10a. From this figure, we can draw two inferences: first, the HyMOFLS method is able to capture the sharp corners relatively accurately than CLSVOF method even for such a coarse mesh resolution; second, the sharp corners of the disk are prone to high change in the curvature and the cell tagging algorithm is appropriately assigning these areas of the interface with MOF interface reconstruction method.

The effect of increasing mesh resolution on the final shape of the notched disk can be seen from Figures 11 and 12 shown for the $128^{2}$ and $256^{2}$ mesh resolutions. The final shapes of the interface become better and regions of the domain with MOF method tagging is reducing with increasing mesh resolution albeit such an observation is natural.

The convergence of the error norms are shown in Figures $13 \mathrm{a}$ and $13 \mathrm{~b}$, It can be observed that the HyMOFLS method consistently yields the error estimate of the same order of magnitude as that of MOF method even for coarse mesh resolutions. Such accuracy of the HyMOFLS is obtained by consuming less average CPU time per time step compared to MOF method even for high mesh resolution (c.f. Figure 13c). Obviously, the CLSVOF method will consume least time due to the absence of computation and advection of phase centroid. Moreover, a second-order convergence of the symmetric and geometric shape errors is observed in Figures $13 \mathrm{a}$ and 13b. Finally, the values of the error norms for the HyMOFLS, MOF, and CLSVOF methods along with the order of convergence (given within parentheses) are given in Table 1 and the average CPU time consumption per timestep in seconds is summarized in Table 2 . 


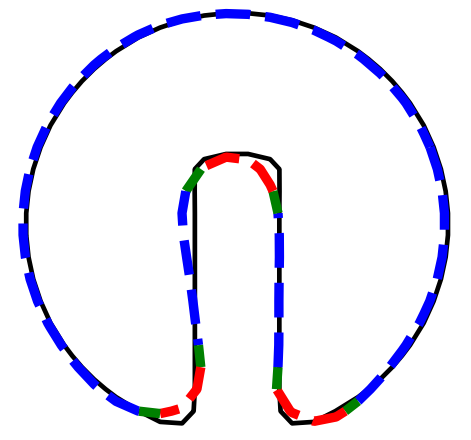

(a) HyMOFLS

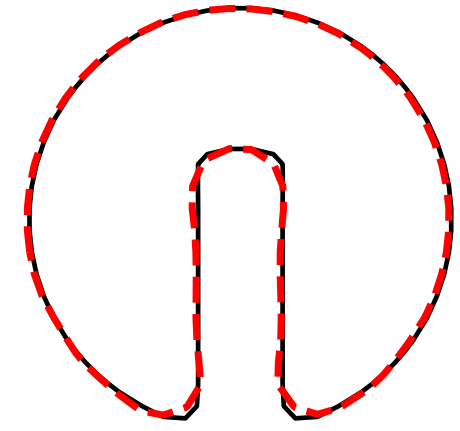

(b) $\mathrm{MOF}$

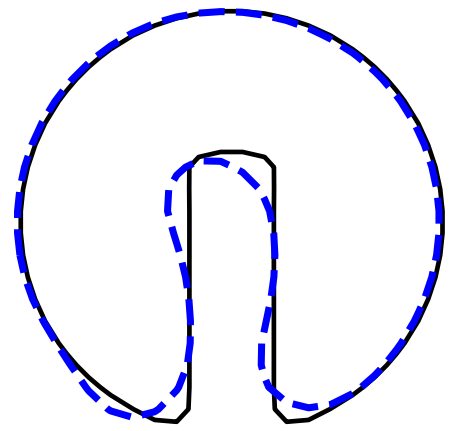

(c) CLSVOF

Figure 10: Phase interface shape of Zalesak's disk after one rotation for $64^{2}$ grid (i.e., $\left.D / \Delta x=19.2\right)$ at initial (solid line) and final (dashed line) time instants. Black color correspond to initial interface while red, blue, and green colors correspond to interface reconstruction using MOF, CLSVOF, and numerical interpolation between MOF and CLSVOF tagged label values, respectively.

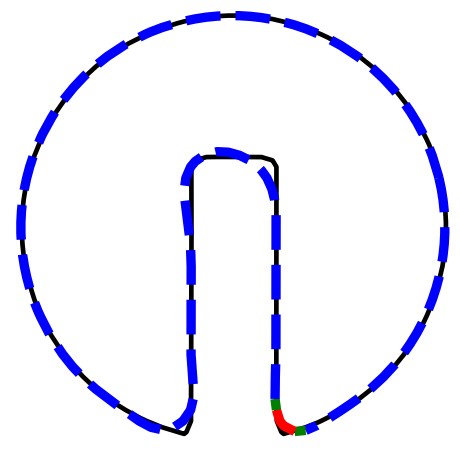

(a) HyMOFLS

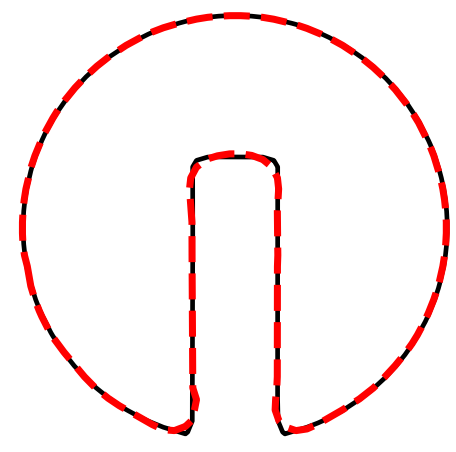

(b) $\mathrm{MOF}$

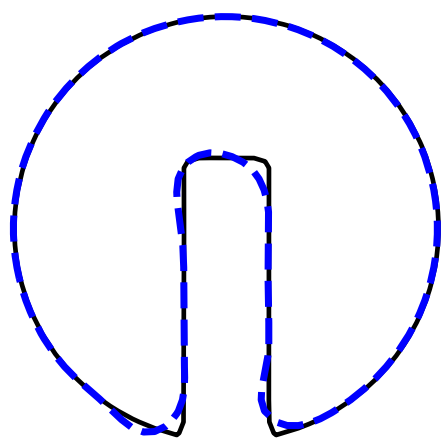

(c) CLSVOF

Figure 11: Phase interface shape of Zalesak's disk after one rotation for $128^{2}$ grid (i.e., $D / \Delta x=38.4$ ) at initial (solid line) and final (dashed line) time instants. Black color correspond to initial interface while red, blue, and green colors correspond to interface reconstruction using MOF, CLSVOF, and numerical interpolation between MOF and CLSVOF tagged label values respectively.

Table 1: Summary of error estimates for Zalesak's disk test with spatial order of error convergence given within parentheses.

\begin{tabular}{|c|c|c|c|c|c|c|}
\hline \multirow[t]{2}{*}{$N_{x}$} & \multicolumn{2}{|c|}{ HyMOFLS } & \multicolumn{2}{|c|}{ MOF } & \multicolumn{2}{|c|}{ CLSVOF } \\
\hline & $E_{\text {symm }}$ & $E_{\text {geo }}$ & $E_{\text {symm }}$ & $E_{\text {geo }}$ & $E_{\text {symm }}$ & $E_{\text {geo }}$ \\
\hline 32 & $4.17 \mathrm{E}-04 \quad(-)$ & $1.92 \mathrm{E}-04 \quad(-)$ & $3.95 \mathrm{E}-04 \quad(-)$ & $1.18 \mathrm{E}-04 \quad(-)$ & $1.65 \mathrm{E}-03 \quad(-)$ & $6.15 \mathrm{E}-04 \quad(-)$ \\
\hline 64 & $6.63 \mathrm{E}-05(2.65)$ & $3.42 \mathrm{E}-05(2.49)$ & $5.99 \mathrm{E}-05(2.72)$ & $1.95 \mathrm{E}-05(2.60)$ & $2.96 \mathrm{E}-04(2.48)$ & $1.13 \mathrm{E}-04(2.44)$ \\
\hline 128 & $1.58 \mathrm{E}-05(2.07)$ & 7.47E-06 (2.20) & $1.53 \mathrm{E}-05(1.97)$ & $6.19 \mathrm{E}-06(1.66)$ & $8.31 \mathrm{E}-05(1.83)$ & $2.52 \mathrm{E}-05(2.17)$ \\
\hline 256 & $3.52 \mathrm{E}-06(2.17)$ & $2.26 \mathrm{E}-06(1.72)$ & $3.21 \mathrm{E}-06(2.25)$ & $1.27 \mathrm{E}-06(2.29)$ & $2.20 \mathrm{E}-05(1.92)$ & $7.32 \mathrm{E}-06(1.78)$ \\
\hline 512 & $9.19 \mathrm{E}-07(1.82)$ & $5.52 \mathrm{E}-07(2.03)$ & $7.42 \mathrm{E}-07(2.11)$ & $2.70 \mathrm{E}-07(2.23)$ & $5.22 \mathrm{E}-06(2.08)$ & $1.41 \mathrm{E}-06(2.38)$ \\
\hline 1024 & $2.22 \mathrm{E}-07(2.05)$ & $1.24 \mathrm{E}-07(2.15)$ & $1.58 \mathrm{E}-07(2.23)$ & $6.01 \mathrm{E}-08(2.17)$ & $1.39 \mathrm{E}-06(1.91)$ & $4.61 \mathrm{E}-07(1.61)$ \\
\hline
\end{tabular}

\subsubsection{D droplet deformation}

The velocity field used for the rotation of notched disk is linear and hence tagging the cells with MOF and CLSVOF methods will be straightforward. In contrast, the numerical simulations of the atomization applications will involve more complex non-linear velocity field (like a turbulent eddy) in which the liquid structure will be present. To that end, as a first step, we test the deformation of a $2 \mathrm{D}$ circular droplet of 


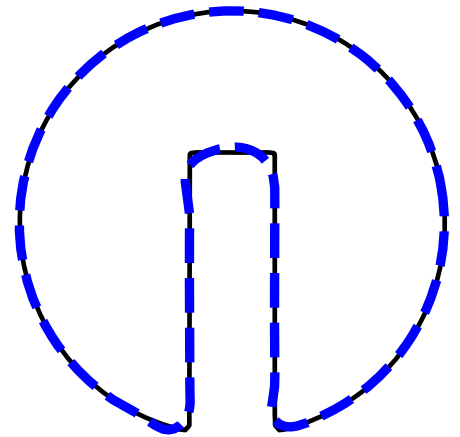

(a) HyMOFLS

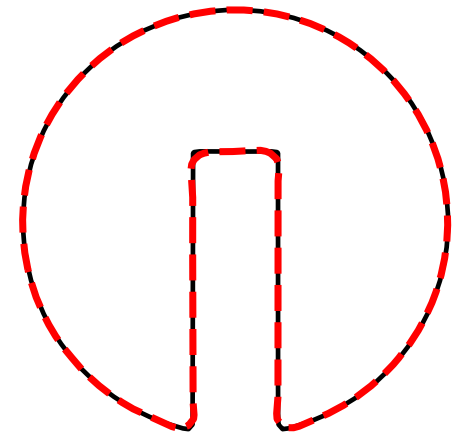

(b) $\mathrm{MOF}$

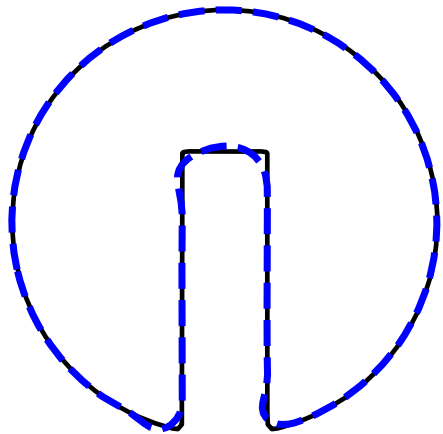

(c) CLSVOF

Figure 12: Phase interface shape of Zalesak's disk after one rotation for $256^{2}$ grid (i.e., $D / \Delta x=76.8$ ) at initial (solid line) and final (dashed line) time instants. Black color correspond to initial interface while red, blue, and green colors correspond to interface reconstruction using MOF, CLSVOF, and numerical interpolation between MOF and CLSVOF tagged label values respectively.

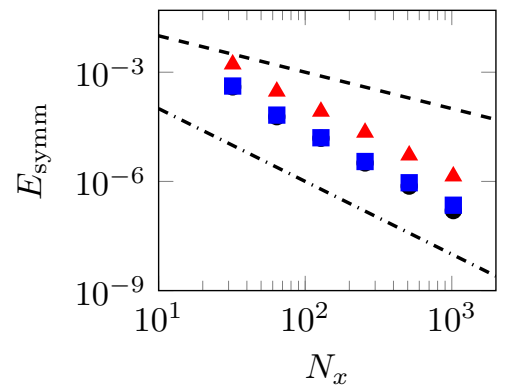

(a) Symmetric difference error

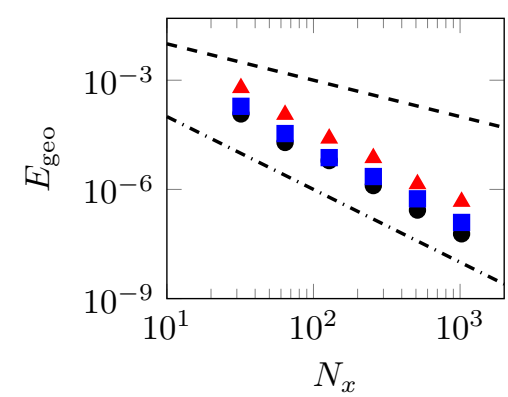

(b) Geometric error

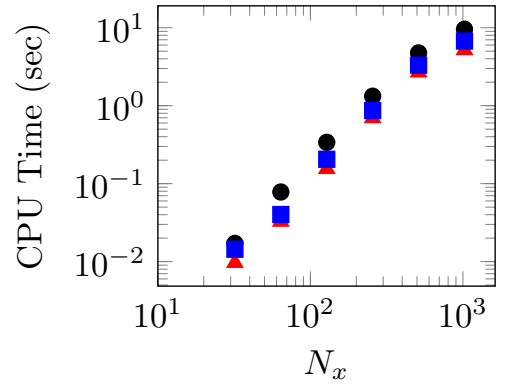

(c) Average CPU time

Figure 13: Error estimates for Zalesak's notched disk rotation test for HyMOFLS (日), MOF (日), and CLSVOF (A) methods along with first-order convergence --- and second-order convergence $-\cdot-\cdot-$ lines.

Table 2: Summary of average CPU time consumption per timestep (in seconds) for Zalesak's disk test.

\begin{tabular}{cccc}
\hline$N_{x}$ & HyMOFLS & MOF & CLSVOF \\
\hline 32 & $1.44 \mathrm{E}-02$ & $1.71 \mathrm{E}-02$ & $9.66 \mathrm{E}-03$ \\
64 & $4.01 \mathrm{E}-02$ & $7.81 \mathrm{E}-02$ & $8.14 \mathrm{E}-03$ \\
128 & $2.05 \mathrm{E}-01$ & $3.39 \mathrm{E}-01$ & $1.55 \mathrm{E}-01$ \\
256 & $8.67 \mathrm{E}-01$ & $1.32 \mathrm{E}+00$ & $6.95 \mathrm{E}-01$ \\
512 & $3.31 \mathrm{E}+00$ & $4.76 \mathrm{E}+00$ & $2.68 \mathrm{E}+00$ \\
1024 & $6.75 \mathrm{E}+00$ & $9.58 \mathrm{E}+00$ & $5.18 \mathrm{E}+00$ \\
\hline
\end{tabular}

liquid under a non-linear velocity field given as

$$
\begin{aligned}
& u=-2 \sin ^{2}(\pi x) \sin (\pi y) \cos (\pi y) \cos (\pi t / T) \\
& v=2 \sin ^{2}(\pi y) \sin (\pi x) \cos (\pi x) \cos (\pi t / T)
\end{aligned}
$$

The droplet has a diameter $D=0.3$ having its center located at $(0.5,0.75)$ inside a $[0,1] \times[0,1]$ domain. In this test, the droplet is subjected to the above non-linear velocity field which progressively entraps it into vortex thereby stretching to small filament-like structure at the moment of maximum deformation and returns back to its original shape at the final time instant. The time period of this test is kept to $T=8$, i.e., the maximum deformation moment is at $t=T / 2=4$ and the final time instant is $t=T=8$. To study the convergence of the error metrics, this test has been carried out for various mesh resolutions ranging 
from $32^{2}$ to $1024^{2}$. The solution obtained on the $1024^{2}$ grid (i.e., $D / \Delta x=307.2$ ) will be used as reference solution against which the results obtained on coarser mesh resolutions are compared.

Figure 14 shows the shape of the interfaces at the maximum deformation time instant and final time instant for HyMOFLS, MOF, and CLSVOF methods for $64^{2}$ mesh resolution (i.e., $D / \Delta x=19.2$ ) with the reference solution (shown in solid black line). By the formulation of the HyMOFLS method, the cells containing the interface are tagged either MOF (red color) or CLSVOF (blue color) method of interface reconstruction. From this figure, it is obvious that the MOF and HyMOFLS methods behave similarly especially when capturing the thin filament-like region at the tail of the stretched shape. Moreover, the interface shape at the final time instant is better with HyMOFLS method than the MOF method. The reason for this super-performance behavior is unclear and requires deeper investigation. The MOF method (i.e., red region) tagged for the tail of the stretched droplet in Figure 14a is an anomaly. This is because this tail region does not experience high curvature but merely due to its under-resolved nature of the liquid structure, the curvature computed using classical nine-point stencil method is leading to a high value since $\|\boldsymbol{\nabla} \phi\|_{2}$ is far from unity. This results in the phenomenon of over-tagging with MOF method. Such a phenomenon can be solved by improving the method of computation of curvature.

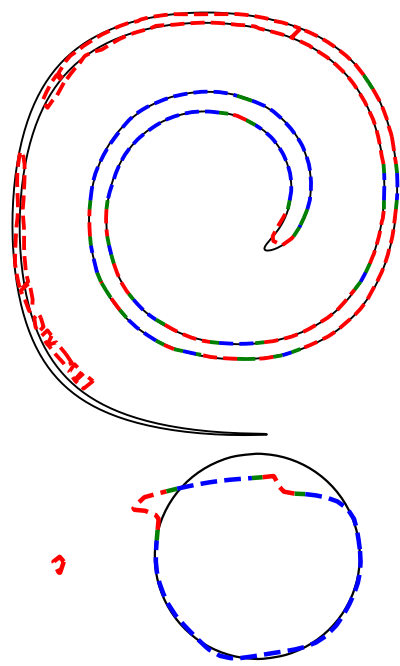

(a) HyMOFLS

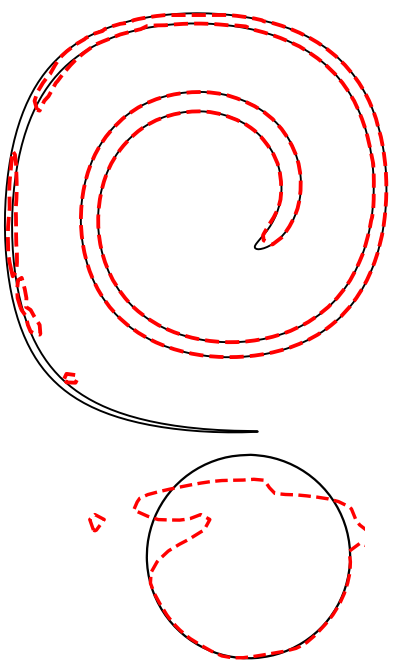

(b) $\mathrm{MOF}$

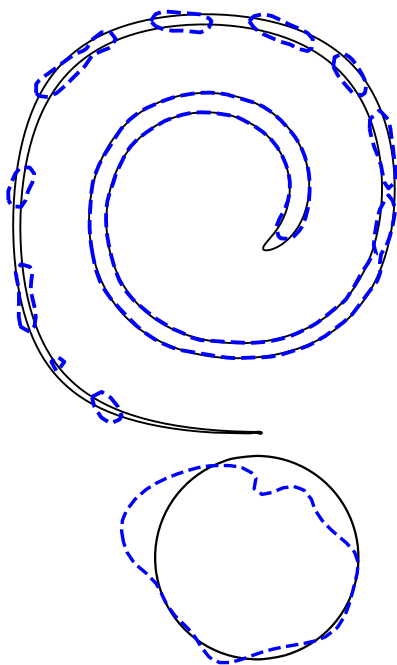

(c) CLSVOF

Figure 14: Phase interface shape of $2 \mathrm{D}$ droplet deformation at maximum deformation (top row) and final time instant (bottom row) for $64^{2}$ grid (i.e., $D / \Delta x=19.2$ ) shown along with reference solution (black line) computed on $1024 \times 1024$ grid. The blue dashed line represent CLSVOF tagged regions, red dashed line represent MOF tagged region, and green dashed line correspond to numerical interpolation between MOF and CLSVOF tagged label values.

The effect of the interface shape and capture of the tail of the deformed droplet with increasing mesh resolution are shown in Figures 15 and 16 for $128^{2}$ and $256^{2}$ mesh resolutions, respectively. It can be seen that not only the tail is progressively better captured but also the interface shape at the final time instant becomes increasingly closer to the reference solution.

The quantitative error metrics and their respective mesh convergence are shown in Figure 17 for this test. From the symmetric difference error shown in Figure 17a, we can see that this error experiences a first-order convergence with increasing mesh resolution. The second-order accuracy of the MOF method is lost due to the first-order of the Eulerian Implicit-Lagrangian Explicit (EI-LE) numerical scheme used for the discretization of the advection equation of the phase centroids (c.f. Equation (22)). However, the convergence of the geometric error metric displays a second-order accuracy since it measures the error in the shape and not the exact error in the interface reconstruction. Moreover, the velocity is non-linear and within the context of reversible test, the errors from the numerical scheme for centroid advection created from $t=0$ to $T / 2$ cancels those from $t=T / 2$ to $T$. Thus, it is unaffected with the numerical scheme 


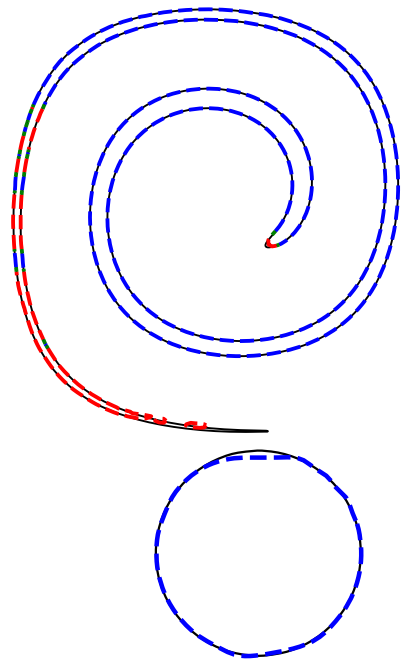

(a) HyMOFLS

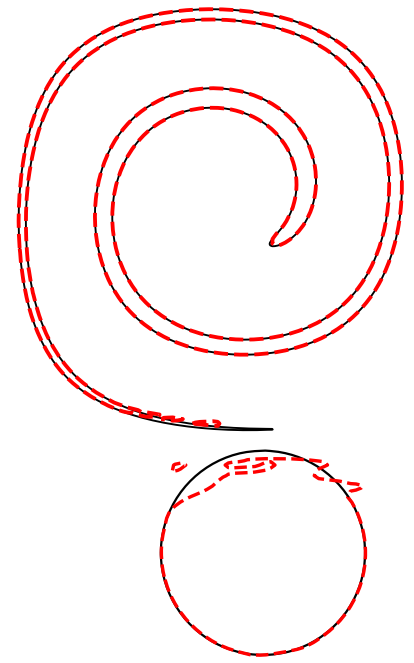

(b) MOF

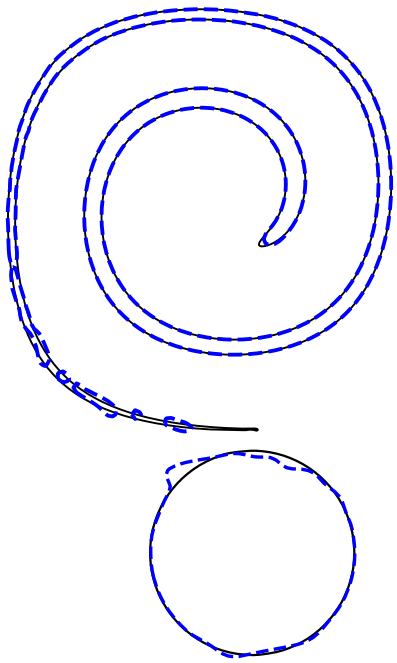

(c) CLSVOF

Figure 15: Phase interface shape of 2D droplet deformation at maximum deformation (top row) and final time instant (bottom row) for $128^{2}$ grid (i.e., $D / \Delta x=38.4$ ) shown along with reference solution (black line) computed on $1024 \times 1024$ grid. The blue dashed line represent CLSVOF tagged regions, red dashed line represent MOF tagged region, and green dashed line correspond to numerical interpolation between MOF and CLSVOF tagged label values.

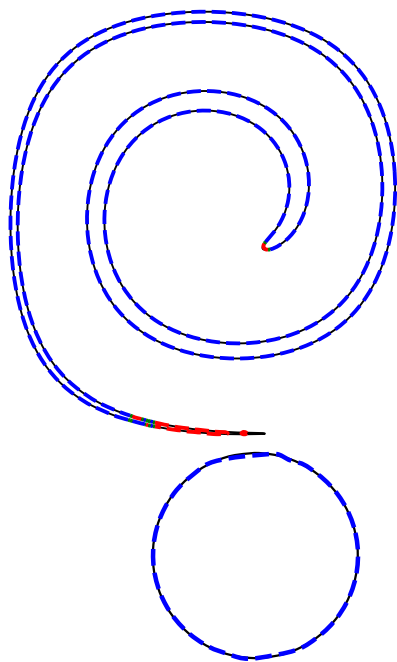

(a) HyMOFLS

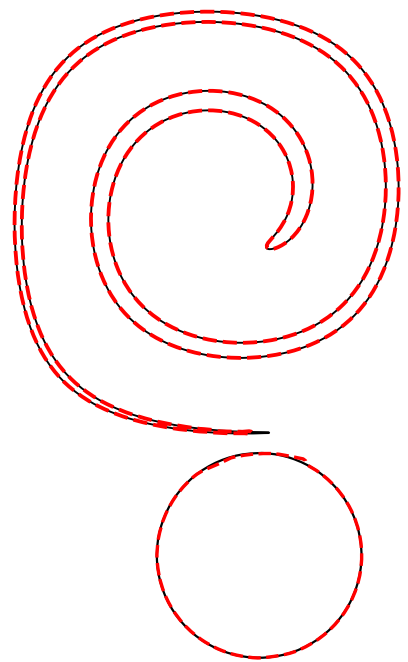

(b) MOF

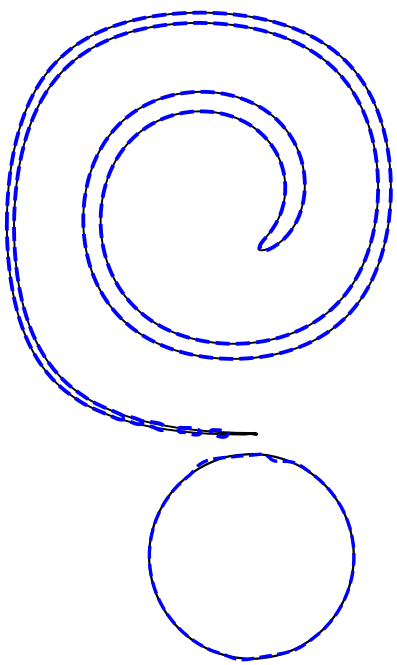

(c) CLSVOF

Figure 16: Phase interface shape of 2D droplet deformation at maximum deformation (top row) and final time instant (bottom row) for $256^{2}$ grid (i.e., $D / \Delta x=76.8$ ) shown along with reference solution (black line) computed on $1024 \times 1024$ grid. The blue dashed line represent CLSVOF tagged regions, red dashed line represent MOF tagged region, and green dashed line correspond to numerical interpolation between MOF and CLSVOF tagged label values.

for the discretization of the advection equation of phase centroid. As expected, the HyMOFLS method is consistently consuming less average CPU time per timestep in comparison to the full MOF method. A summary of the error norms along with their order of convergence (given within parentheses) for HyMOFLS, MOF, and CLSVOF methods are listed in Table 3.

The advantage of the HyMOFLS method is that the MOF method is used only when it is necessary. The high computational expense of the MOF method is directly associated to the number of calls to the Gauss- 


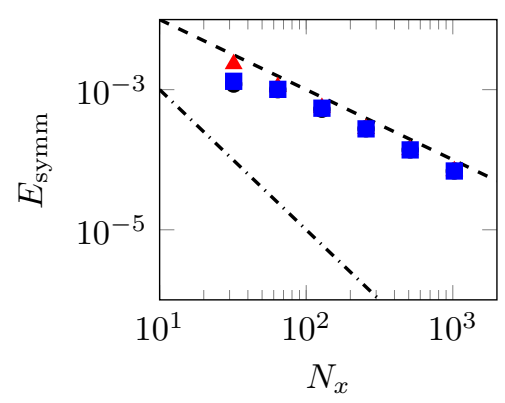

(a) Symmetric difference error

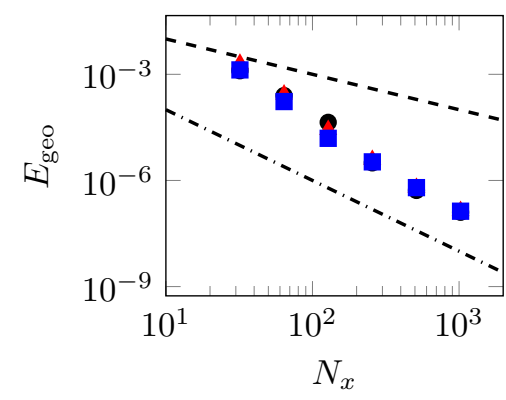

(b) Geometric error

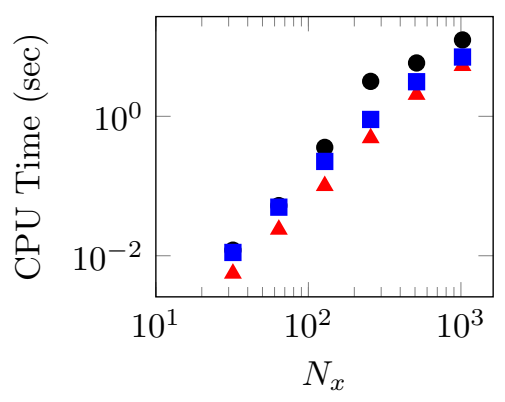

(c) Average CPU time

Figure 17: Error estimates for 2D droplet deformation test for HyMOFLS first-order convergence --- and second-order convergence ---- lines.

Table 3: Summary of error estimates for 2D droplet deformation test with spatial order of error convergence given within parentheses.

\begin{tabular}{|c|c|c|c|c|c|c|}
\hline \multirow[t]{2}{*}{$N_{x}$} & \multicolumn{2}{|c|}{ HyMOFLS } & \multicolumn{2}{|c|}{ MOF } & \multicolumn{2}{|c|}{ CLSVOF } \\
\hline & $E_{\text {symm }}$ & $E_{\mathrm{geo}}$ & $E_{\text {symm }}$ & $E_{\text {geo }}$ & $E_{\text {symm }}$ & $E_{\text {geo }}$ \\
\hline 32 & $1.32 \mathrm{E}-03 \quad(-)$ & $1.33 \mathrm{E}-03 \quad(-)$ & $1.20 \mathrm{E}-03 \quad(-)$ & $1.27 \mathrm{E}-03 \quad(-)$ & $2.32 \mathrm{E}-03 \quad(-)$ & $2.02 \mathrm{E}-03 \quad(-)$ \\
\hline 64 & $1.01 \mathrm{E}-03(0.39)$ & $1.70 \mathrm{E}-04(2.97)$ & $9.97 \mathrm{E}-04(0.27)$ & $2.51 \mathrm{E}-04(2.34)$ & $1.10 \mathrm{E}-03(1.08)$ & $2.71 \mathrm{E}-04(2.90)$ \\
\hline 128 & $5.44 \mathrm{E}-04(0.89)$ & $1.55 \mathrm{E}-05(3.46)$ & $5.25 \mathrm{E}-04(0.93)$ & $4.39 \mathrm{E}-05(2.52)$ & $5.52 \mathrm{E}-04(0.99)$ & $2.70 \mathrm{E}-05(3.33)$ \\
\hline 256 & $2.76 \mathrm{E}-04(0.98)$ & $3.36 \mathrm{E}-06(2.21)$ & $2.76 \mathrm{E}-04(0.93)$ & $3.15 \mathrm{E}-06(3.80)$ & $2.76 \mathrm{E}-04(1.00)$ & $3.85 \mathrm{E}-06(2.81)$ \\
\hline 512 & $1.38 \mathrm{E}-04(1.00)$ & $6.27 \mathrm{E}-07(2.42)$ & $1.38 \mathrm{E}-04(1.00)$ & $5.23 \mathrm{E}-07(2.59)$ & $1.38 \mathrm{E}-04(1.00)$ & $6.22 \mathrm{E}-07(2.63)$ \\
\hline 1024 & $6.90 \mathrm{E}-05(1.00)$ & $1.35 \mathrm{E}-07(2.22)$ & $6.90 \mathrm{E}-05(1.00)$ & $1.26 \mathrm{E}-07(2.05)$ & $6.90 \mathrm{E}-05(1.00)$ & $1.38 \mathrm{E}-07(2.17)$ \\
\hline
\end{tabular}

Table 4: Summary of average CPU time consumption per timestep (in seconds) for 2D droplet deformation test.

\begin{tabular}{cccc}
\hline$N_{x}$ & HyMOFLS & MOF & CLSVOF \\
\hline 32 & $1.12 \mathrm{E}-02$ & $1.20 \mathrm{E}-02$ & $5.60 \mathrm{E}-03$ \\
64 & $4.98 \mathrm{E}-02$ & $5.24 \mathrm{E}-02$ & $2.38 \mathrm{E}-02$ \\
128 & $2.25 \mathrm{E}-01$ & $3.58 \mathrm{E}-01$ & $1.01 \mathrm{E}-01$ \\
256 & $9.02 \mathrm{E}-01$ & $3.17 \mathrm{E}+00$ & $4.88 \mathrm{E}-01$ \\
512 & $3.14 \mathrm{E}+00$ & $5.83 \mathrm{E}+00$ & $2.05 \mathrm{E}+00$ \\
1024 & $7.09 \mathrm{E}+00$ & $12.46 \mathrm{E}+00$ & $5.30 \mathrm{E}+00$ \\
\hline
\end{tabular}

Newton algorithm (c.f. Algorithm 1). This is because four interface reconstructions are performed in this algorithm to compute the derivative of the objective function before finally determining the optimal values of the components of interface unit normal. Therefore, in order to evaluate the step-up that that was obtained from HyMOFLS method, we now present in Table 5 the comparison between the MOF and HyMOFLS methods in terms of the total number of Gauss-Newton algorithm calls, total number of iterations, and average number of iterations per Gauss-Newton call for this test using $64^{2}$ mesh resolution. The total CPU time taken for the MOF method is $221.41 \mathrm{~s}$ while for the HyMOFLS method is $185.98 \mathrm{~s}$ each using 4 processors run in Myria supercomputer at CRIANN 60. It can be clearly seen that the HyMOFLS takes lesser number of Gauss-Newton iterations and lesser average CPU time consumption than that for the MOF method emphasizing the fruitfulness of this hybrid framework. However, it is to be remarked that the average number of iterations obtained from our implementation of MOF and HyMOFLS method is observed to be taking more number of Gauss-Newton iterations for the convergence in computation of the actual centroid $x_{\mathrm{COM}}^{\text {act }}$ and $E_{\mathrm{MOF}}$ than the study by Jemison et al. [27. This goes to show that the estimation of our initial guess for the MOF method can be improved. 
Table 5: Summary of total number of Gauss-Newton calls and iterations for interface reconstruction for MOF and HyMOFLS methods for $2 \mathrm{D}$ droplet deformation test using $64^{2}$ mesh resolution.

\begin{tabular}{lcccc}
\hline Metric of measurement & \multicolumn{2}{c}{ MOF } & \multicolumn{2}{c}{ HyMOFLS } \\
\cline { 2 - 4 } & $t=4.0$ & $t=8.0$ & $t=4.0$ & $t=8.0$ \\
\hline Total number of Gauss-Newton calls & 2214 & 493 & 952 & 65 \\
Total number of iterations for convergence & 3549 & 1317 & 2060 & 256 \\
Average number of iterations per call & 1.60 & 2.67 & 2.16 & 3.94 \\
\hline
\end{tabular}

\subsubsection{D droplet deformation}

The test of the non-linear velocity on the interface reconstruction is now extended to three dimensions. In this test 61 , a spherical droplet of diameter $D=0.3$ is placed at $(0.35,0.35,0.35)$ within a $[0,1] \times[0,1] \times[0,1]$ domain. This droplet is now subjected to the following velocity field 62 .

$$
\begin{array}{r}
u(x, y, z, t)=2 \sin ^{2}(\pi x) \sin (2 \pi y) \sin (2 \pi z) \cos (\pi t / 3) \\
v(x, y, z, t)=-\sin (2 \pi x) \sin ^{2}(\pi y) \sin (2 \pi z) \cos (\pi t / 3) \\
w(x, y, z, t)=-\sin (2 \pi x) \sin (2 \pi y) \sin ^{2}(\pi z) \cos (\pi t / 3)
\end{array}
$$

which stretches the interface to form a thin membrane at the time instant of maximum deformation $(t=T / 2)$ and the interface comes back to its spherical shape at the final time instant of $t=T=3$. The objectives of this test are to capture the thin membrane of the stretched sphere at $t=T / 2$ and to recover the spherical shape of the droplet at $t=T$. In order to study the convergence rate of the error metrics, six mesh resolutions ranging from $32^{3}$ to $512^{3}$ including $192^{3}$ have been utilized to discretize the domain.

Figure 18 shows the phase interface of the $3 \mathrm{D}$ droplet at the maximum deformation time instant (top row) and the final time instant (bottom row) for $192^{3}$ mesh resolution. The red regions indicate the MOF method tagged regions while the blue regions indicate the CLSVOF method tagged region. The following inferences are drawn from this figure: first, all the three interface reconstruction methods are able to capture the flat thin membranes in the maximum deformed spheres (see Section 2.4.3) using this mesh resolution at the time instant of maximum deformation; second, the HyMOFLS method is producing relatively more spherical shape at the final time instant with less surface deformation than the MOF and CLSVOF method; and third, HyMOFLS method is using MOF at the exact regions where there is high change in the curvature and at the thin membrane region. However, the flat thin membrane with small curvature value with thickness spanning 4 computational cells is tagged with MOF method. This MOF tagging (called over-tagging) is unnecessary and is occurring as the curvature is not well computed in ARCHER when the interface spans over few cells (in this case 4 cells) resulting in value of IRQ for these cells satisfying the under-resolved criterion. When the curvature computation is improved, it is envisaged that this thin membrane will be tagged entirely with CLSVOF method thereby reducing the total CPU time even further.

In order to emphasize the importance of the mesh resolution on the interface capture, Figures 19 and 20 show the phase interface for the $128^{3}(D / \Delta x=38.4)$ and $256^{3}(D / \Delta x=76.8)$ mesh resolutions. It can be seen that for a coarse mesh resolution of $128^{3}$, there are no significant differences in the phase interface shape at the maximum deformation time instant between HyMOFLS and MOF method. And as the mesh resolution is doubled to $256^{3}$, the final shape tends to be more spherical with less surface deformations.

With the qualitative comparisons shown above, we now present the quantitative comparisons of the results among HyMOFLS, MOF, and CLSVOF methods. To that end, the error metrics as well as the average CPU time consumption are shown in Figure 21. From this figure, we can see that both the symmetric difference error and geometric shape error approximately follows the second-order convergence rate with respect to the spatial mesh resolution. And the average CPU time consumed per timestep of the iteration in the test is consistently lower for the HyMOFLS method compared to the MOF method. One more observation is that this average CPU time consumption for the HyMOFLS method tend towards that for the CLSVOF method as seen in Figure 21c. This is because with the increase in mesh resolution, the interface becomes increasingly well resolved thereby leading to increase in the number of cells tagged with CLSVOF 


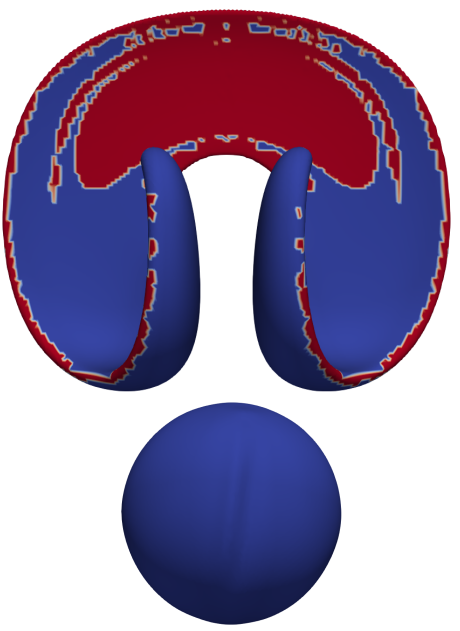

(a) HyMOFLS

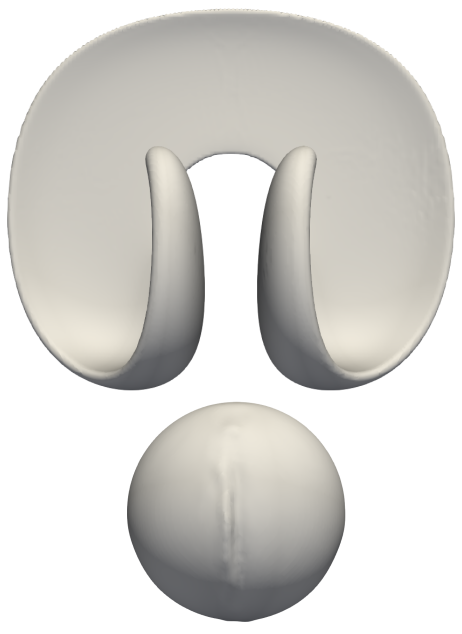

(b) $\mathrm{MOF}$

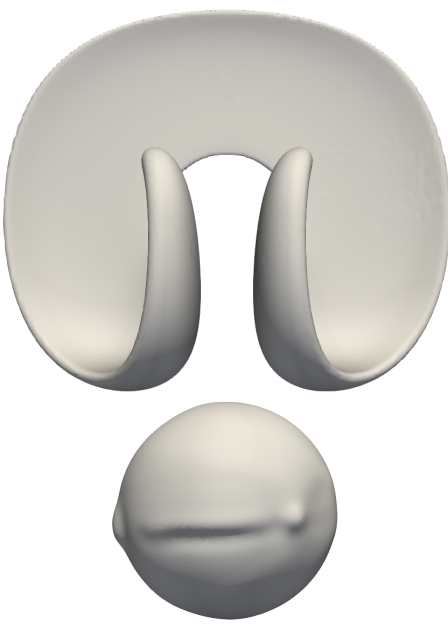

(c) CLSVOF

Figure 18: Phase interface shape of 3D droplet deformation at maximum deformation (top row) and final time instant (bottom row) for $192^{3}$ grid (i.e., $\left.D / \Delta x=57.6\right)$. Red region represent MOF method tagged region and blue region represent CLSVOF method tagged region.

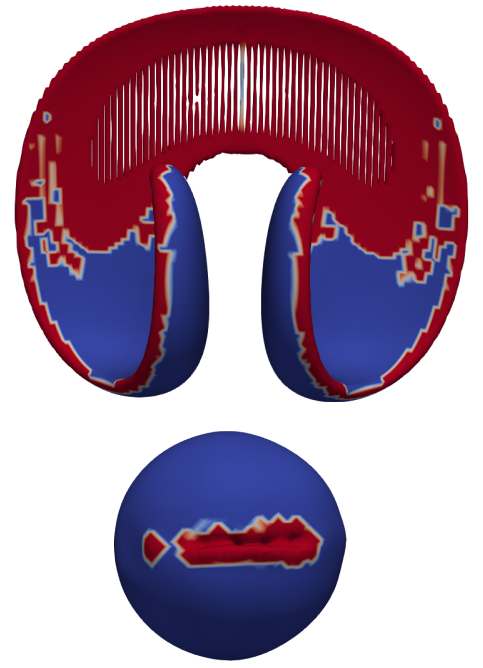

(a) HyMOFLS
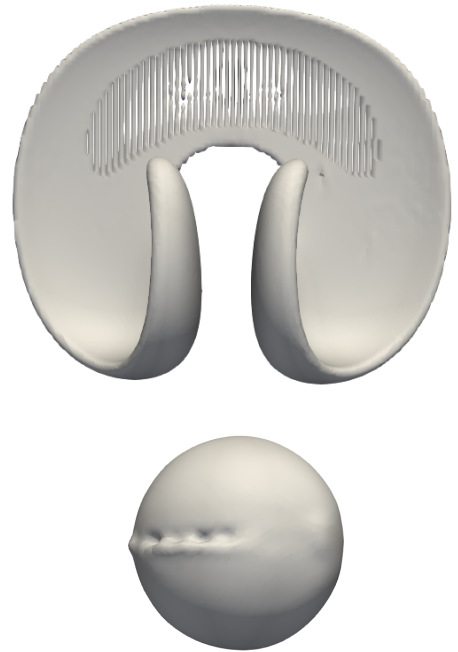

(b) $\mathrm{MOF}$
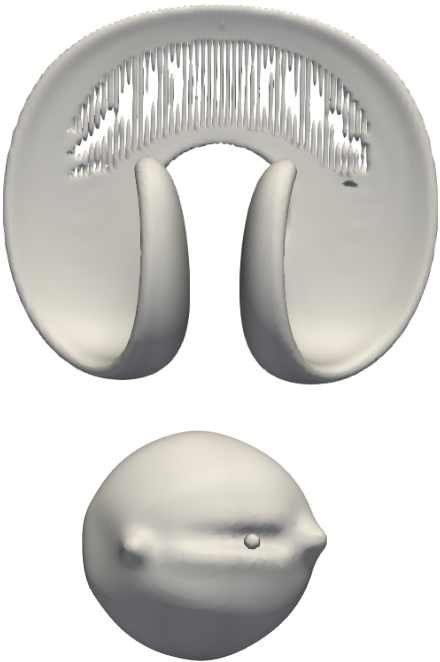

(c) CLSVOF

Figure 19: Phase interface shape of 3D droplet deformation at maximum deformation (top row) and final time instant (bottom row) for $128^{3}$ grid (i.e., $D / \Delta x=38.4$ ). Red region represent MOF method tagged region and blue region represent CLSVOF method tagged region.

method and hence, the HyMOFLS method virtually becomes CLSVOF method. The error estimate values for various mesh resolutions pertaining to HyMOFLS, MOF, and CLSVOF method are summarized in Table 6 with the error convergence order given within parentheses.

Finally, in order to evaluate the step-up obtained by combining MOF with CLSVOF method within the HyMOFLS framework, we now present in Table 8 the comparison between the MOF and HyMOFLS methods in terms of the total number of Gauss-Newton algorithm calls, total number of iterations, and average number of iterations per Gauss-Newton call for this test using $64^{3}$ mesh resolution. The total CPU time taken for the MOF method is $2848.77 \mathrm{~s}$ and for the HyMOFLS method is $2106.38 \mathrm{~s}$ on 8 processors 


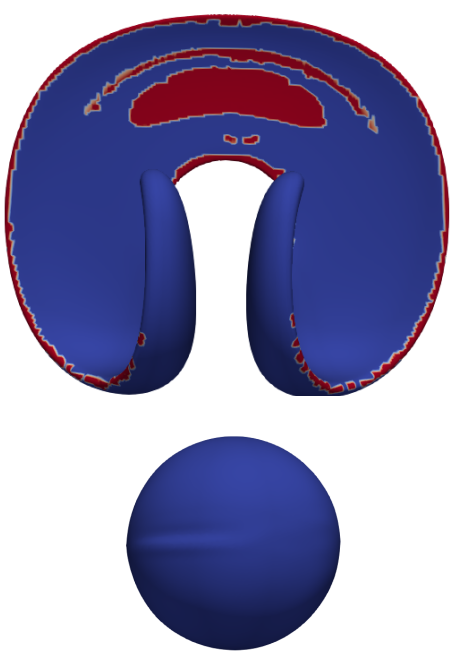

(a) HyMOFLS

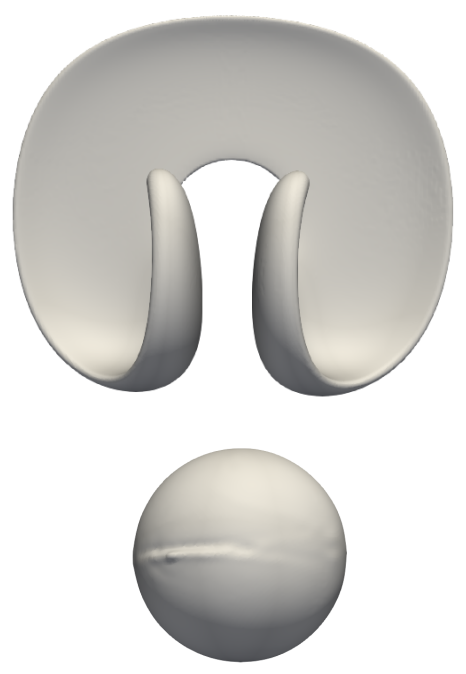

(b) $\mathrm{MOF}$

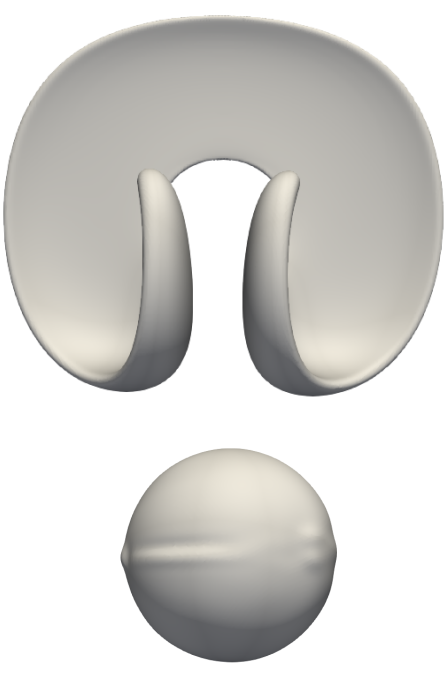

(c) CLSVOF

Figure 20: Phase interface shape of 3D droplet deformation at maximum deformation (top row) and final time instant (bottom row) for $256^{3}$ grid (i.e., $D / \Delta x=76.8$ ). Red region represent MOF method tagged region and blue region represent CLSVOF method tagged region.

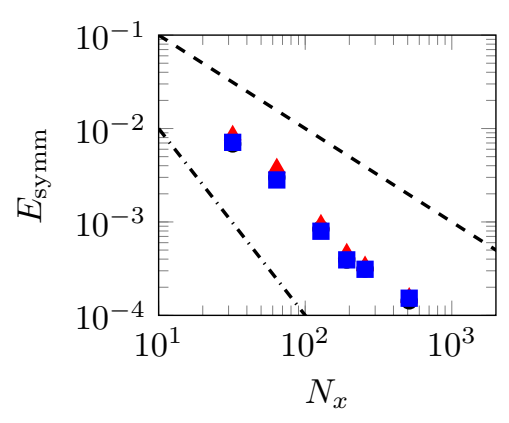

(a) Symmetric difference error

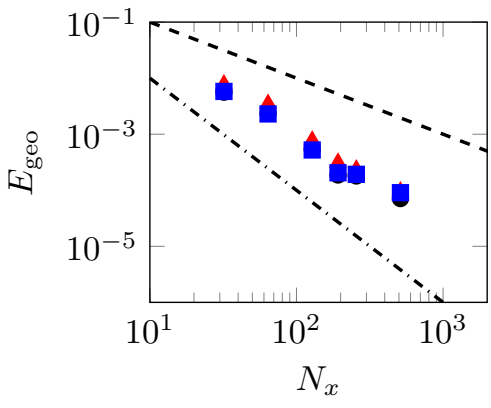

(b) Geometric error

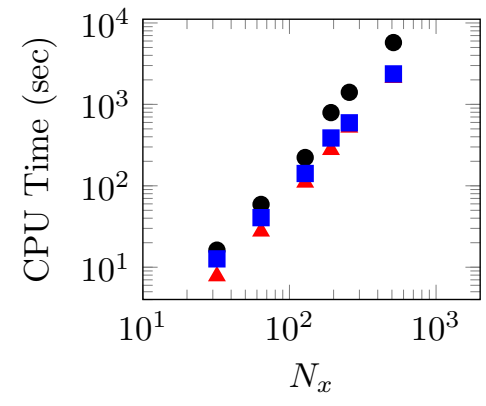

(c) Average CPU time

Figure 21: Error estimates for 3D droplet deformation test for HyMOFLS $(\boldsymbol{\nabla})$, MOF $(\boldsymbol{\theta}$, and CLSVOF $\boldsymbol{\Theta}$ methods along with first-order convergence --- ; second-order convergence ---- lines.

Table 6: Summary of error estimates for 3D droplet deformation test with spatial order of error convergence given within parentheses.

\begin{tabular}{|c|c|c|c|c|c|c|}
\hline \multirow[t]{2}{*}{$N_{x}$} & \multicolumn{2}{|c|}{ HyMOFLS } & \multicolumn{2}{|c|}{ MOF } & \multicolumn{2}{|c|}{ CLSVOF } \\
\hline & $E_{\text {symm }}$ & $E_{\text {geo }}$ & $E_{\text {symm }}$ & $E_{\text {geo }}$ & $E_{\mathrm{symm}}$ & $E_{\text {geo }}$ \\
\hline 32 & $7.12 \mathrm{E}-03 \quad(-)$ & $5.80 \mathrm{E}-03 \quad(-)$ & $6.83 \mathrm{E}-03 \quad(-)$ & $5.62 \mathrm{E}-03 \quad(-)$ & $8.52 \mathrm{E}-03 \quad(-)$ & $7.77 \mathrm{E}-03 \quad(-)$ \\
\hline 64 & $2.82 \mathrm{E}-03(1.34)$ & $2.30 \mathrm{E}-03(1.57)$ & $2.99 \mathrm{E}-03(1.19)$ & $2.35 \mathrm{E}-03(1.26)$ & $3.78 \mathrm{E}-03(1.17)$ & $3.50 \mathrm{E}-03(1.15)$ \\
\hline 128 & $7.96 \mathrm{E}-04(1.83)$ & $5.23 \mathrm{E}-04(2.14)$ & $8.38 \mathrm{E}-04(1.84)$ & $5.38 \mathrm{E}-04(2.13)$ & $9.47 \mathrm{E}-04(1.99)$ & $7.72 \mathrm{E}-04(2.18)$ \\
\hline 192 & $3.93 \mathrm{E}-04(1.74)$ & 2.05E-04 (2.31) & $3.91 \mathrm{E}-04(1.88)$ & $1.87 \mathrm{E}-04(2.60)$ & $4.64 \mathrm{E}-04(1.83)$ & $3.12 \mathrm{E}-04(2.23)$ \\
\hline 256 & $3.11 \mathrm{E}-04(1.36)$ & $1.92 \mathrm{E}-04(1.45)$ & $3.14 \mathrm{E}-04(1.42)$ & $1.80 \mathrm{E}-04(1.48)$ & $3.39 \mathrm{E}-04(1.50)$ & $2.35 \mathrm{E}-04(1.72)$ \\
\hline 512 & $1.53 \mathrm{E}-04(1.02)$ & $9.05 \mathrm{E}-05(1.09)$ & $1.43 \mathrm{E}-04(1.14)$ & $7.11 \mathrm{E}-05(1.12)$ & $1.56 \mathrm{E}-04(1.12)$ & $9.56 \mathrm{E}-05(1.30)$ \\
\hline
\end{tabular}

run in Myria supercomputer at CRIANN [60. It can be seen again that the HyMOFLS method takes less number of Gauss-Newton iteration to minimize the centroid error in interface reconstruction. 
Table 7: Summary of average CPU time consumption per timestep (in seconds) for 3D droplet deformation test.

\begin{tabular}{cccc}
\hline$N_{x}$ & HyMOFLS & MOF & CLSVOF \\
\hline 32 & $1.27 \mathrm{E}+01$ & $1.62 \mathrm{E}+01$ & $7.80 \mathrm{E}+00$ \\
64 & $4.06 \mathrm{E}+01$ & $5.91 \mathrm{E}+01$ & $2.76 \mathrm{E}+01$ \\
128 & $1.42 \mathrm{E}+02$ & $2.23 \mathrm{E}+02$ & $1.10 \mathrm{E}+02$ \\
256 & $3.87 \mathrm{E}+02$ & $7.92 \mathrm{E}+02$ & $2.77 \mathrm{E}+02$ \\
512 & $5.94 \mathrm{E}+02$ & $1.41 \mathrm{E}+03$ & $5.21 \mathrm{E}+02$ \\
1024 & $2.37 \mathrm{E}+03$ & $5.74 \mathrm{E}+03$ & $2.12 \mathrm{E}+03$ \\
\hline
\end{tabular}

Table 8: Summary of total number of Gauss-Newton calls and iterations for interface reconstruction for MOF and HyMOFLS methods for $3 \mathrm{D}$ droplet deformation test using $64^{3}$ mesh resolution.

\begin{tabular}{lcccc}
\hline Metric of measurement & \multicolumn{2}{c}{ MOF } & \multicolumn{2}{c}{ HyMOFLS } \\
\cline { 2 - 5 } & $t=1.5$ & $t=3.0$ & $t=1.5$ & $t=3.0$ \\
\hline Total number of Gauss-Newton calls & 40931 & 17052 & 17492 & 4860 \\
Total number of iterations for convergence & 195580 & 83335 & 90965 & 30548 \\
Average number of iterations per call & 4.78 & 4.89 & 5.20 & 6.29 \\
\hline
\end{tabular}

\subsection{Parametric study}

The HyMOFLS method consists of tagging computational cells with MOF and CLSVOF method based on the IRQ URLS/RLS criterion (c.f. Equation (32)) which employs a threshold value $\alpha$. This subsection presents a parametric study on the value of $\alpha$ and highlights the choice of $\alpha=2$ employed in the numerical tests presented above. To that end, we recall the criterion for tagging cell with MOF method (for cells belonging to URLS) and CLSVOF method (for cells belonging to resolved liquid structures) in Algorithm 5 where $i, j$, and $k$ are the indices of the computational cell. This IRQ criterion exploits the value of local

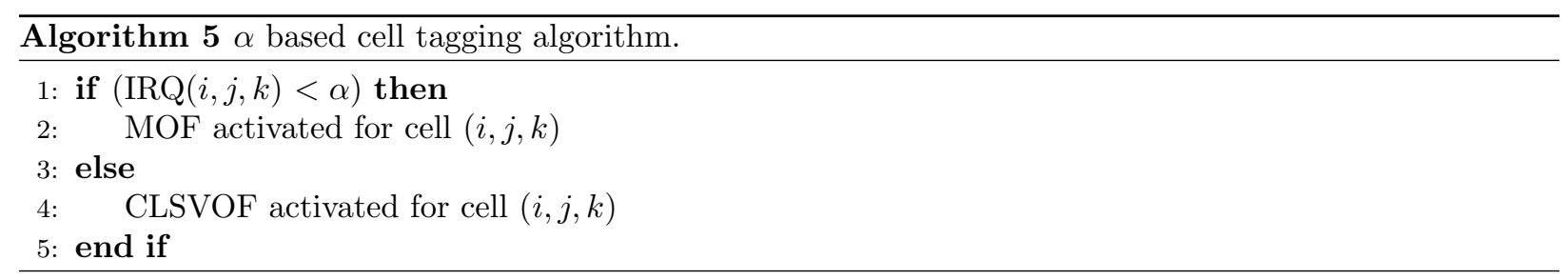

interface curvature and local mesh spacing. However, at times, when the computation of curvature is not highly accurate, there arises a situation that a cell is wrongly tagged as MOF cell leading to a scenario called over-tagging. Such a scenario has been demonstrated in Sections 2.4.2 and 2.4.3 for the 2D and 3D droplet deformation tests, respectively. On one hand, the 2D test revealed that the tail of the stretched droplet spanning few cells was assigned to MOF method even though the curvature of this structure is not very high. One the other hand, the 3D test showed light on the fact that even though the stretched membrane having low curvature values is spanning few cells, the MOF method is being used to reconstruct its interfacial area. One way to mitigate this issue is to have an another criterion $\left|\|\nabla \phi(i, j, k)\|_{2}-1\right|>\delta$ (where $\phi$ is the level set function) in addition to the IRQ criterion for the cell tagging algorithm. The value of $\delta$ can be chosen appropriately similar to the procedure described for $\alpha$ in the following subsection. The investigation of this combined criterion is beyond the scope of this work and is not presented here. However, the IRQ criterion is able to detect the thin structures as shown in Sections 2.4.2 and 2.4.3.

With this premise, we now present the parametric study of $\alpha$ for the interface reconstruction in $2 \mathrm{D}$ and $3 \mathrm{D}$ droplet deformation tests. To that end, we have chosen $64^{2}$ and $64^{3}$ mesh resolutions for respective tests to be performed using HyMOFLS method for the values of $\alpha=0.5,1,2,6$. 


\subsection{1. $2 D$ droplet deformation}

First, we find the effect of $\alpha$ on the results of interface reconstruction for $2 \mathrm{D}$ droplet deformation test. Figure 22 present the results with the shapes of the phase interface along with the result for $\alpha=2$ which was also shown in Figure 14. By qualitative inspection, we find that the result for $\alpha=2$ is matching relatively well with the reference results than for all the other $\alpha$ values.

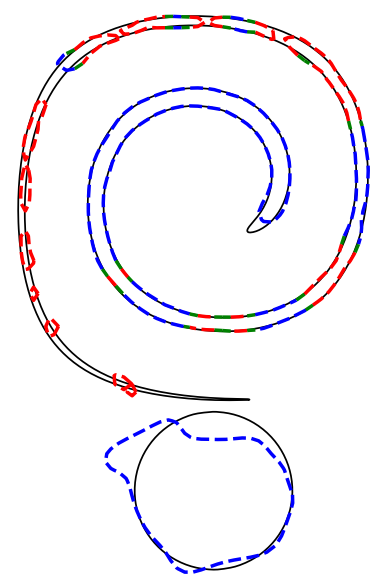

(a) $\alpha=0.5$

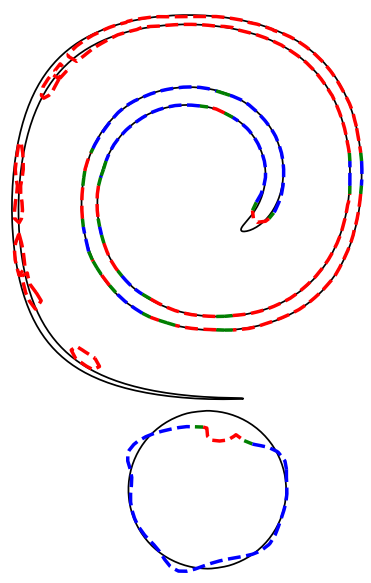

(b) $\alpha=1.0$

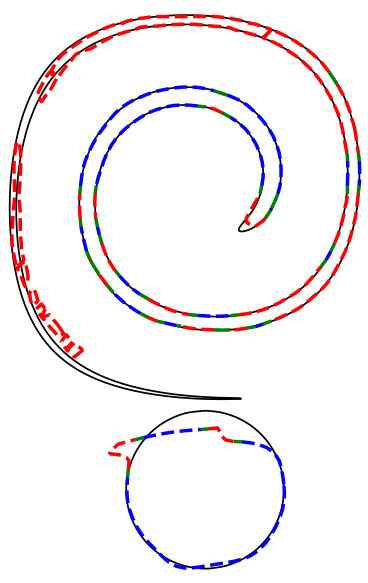

(c) $\alpha=2.0$

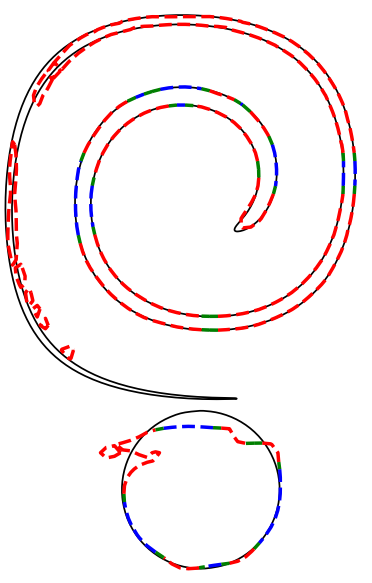

(d) $\alpha=6.0$

Figure 22: Effect of $\alpha$ on the phase interface shape for $2 \mathrm{D}$ droplet deformation test using $64^{2}$ grid (i.e., $D / \Delta x=19.2$ ) shown along with reference solution (black line) computed on $1024 \times 1024$ grid. Blue dashed line represent CLSVOF tagged regions, red dashed line represent MOF tagged regions, and green dashed line correspond to numerical interpolation between MOF and CLSVOF tagged label values.

The plot of the evolution of the error norms as a function of $\alpha$ is shown in Figure 23 with their values summarized in Table 9. Upon quantitative comparison of the error norms for the various values of $\alpha$, we see that the $\alpha=1,2,6$ give approximately the same error. In fact, it can be seen that the error remains constant for all values of $\alpha \geq 1$. This test was not conclusive in determining the exact value of $\alpha$ to be used, hence, we proceed to do the test for the $3 \mathrm{D}$ droplet deformation.

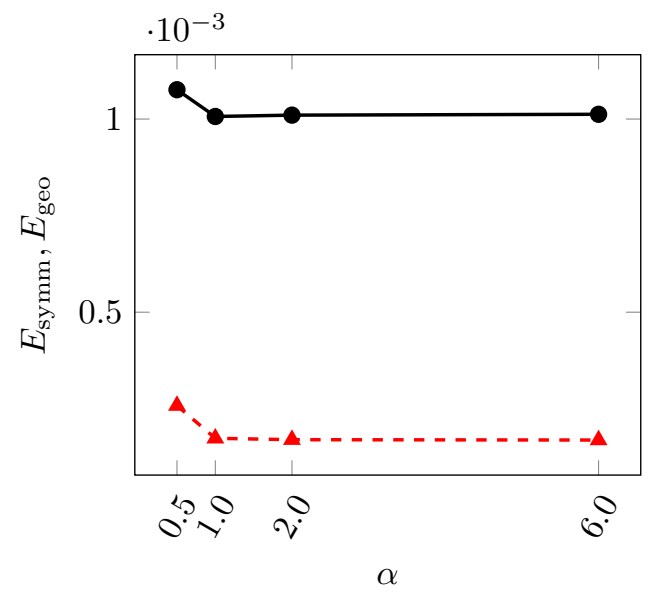

Figure 23: Variation of error norms $\left(E_{\mathrm{symm}}\right.$ : $E_{\text {geo }}:-\underline{\Delta-}$ with $\alpha$ for $2 \mathrm{D}$ droplet deformation test. 
Table 9: Summary of error norms for various $\alpha$ values for parametric study within the framework of HyMOFLS method for $2 \mathrm{D}$ droplet deformation test.

\begin{tabular}{|c|c|c|c|c|}
\hline Error Norm $\alpha$ & 0.5 & 1.0 & 2.0 & 6.0 \\
\hline$E_{\text {symm }}$ & $1.08 \mathrm{E}-03$ & $1.02 \mathrm{E}-03$ & $1.01 \mathrm{E}-03$ & $1.01 \mathrm{E}-03$ \\
\hline$E_{\text {geo }}$ & $2.58 \mathrm{E}-04$ & $1.74 \mathrm{E}-04$ & $1.70 \mathrm{E}-04$ & $1.69 \mathrm{E}-04$ \\
\hline
\end{tabular}

\subsubsection{D droplet deformation}

Next, we apply the same method of analysis to the 3D droplet deformation test. The shapes of the phase interface of the droplets at the mid and the final time instant are shown in Figure 24 for different values of $\alpha$. The plot of variation of error norms as a function of $\alpha$ is shown in Figure 25 while their values are

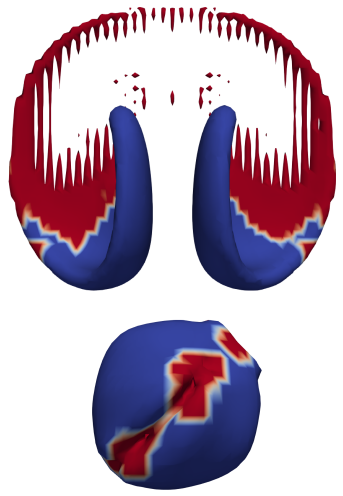

(a) $\alpha=0.5$

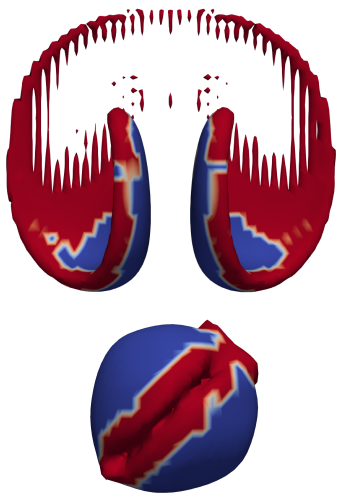

(b) $\alpha=1.0$

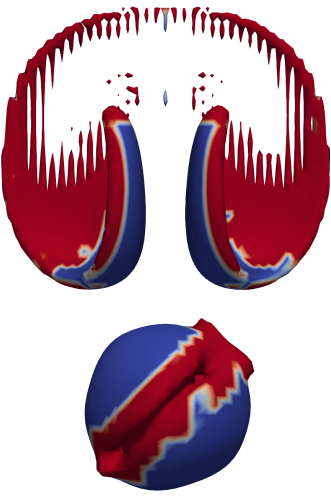

(c) $\alpha=2.0$

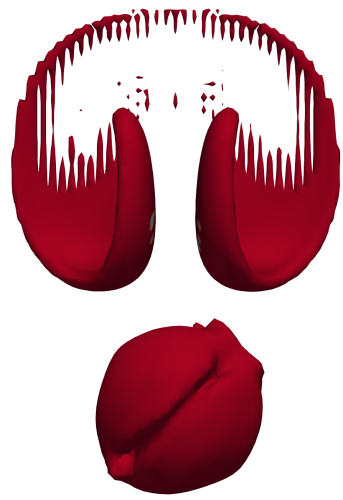

(d) $\alpha=6.0$

Figure 24: Effect of $\alpha$ on the phase interface shape for 3D droplet deformation test using $64^{3}$ grid (i.e., $D / \Delta x=19.2$ ). Red region represent MOF method labeled region and blue region represent CLSVOF method labeled region.

summarized in Table 10

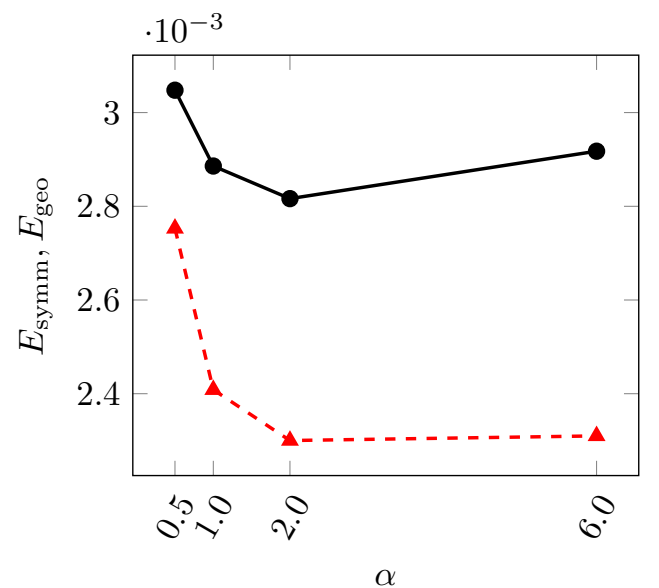

Figure 25: Variation of error norms $\left(E_{\mathrm{symm}}\right.$

$E_{\text {geo }}:$

Evidently, it can be seen that there are no significant differences in the interface shapes for all values of $\alpha$. However, it is unsurprising to see the increase in the amount of MOF method usage (red colored regions) in the interface with this increase in $\alpha$ value. In terms of quantitative comparison, it is apparent that the 
Table 10: Summary of error norms for various $\alpha$ values for parametric study within the framework of HyMOFLS method for 3D droplet deformation test.

\begin{tabular}{|c|c|c|c|c|}
\hline Error Norm $\alpha$ & 0.5 & 1.0 & 2.0 & 6.0 \\
\hline$E_{\text {symm }}$ & $3.05 \mathrm{E}-03$ & $2.89 \mathrm{E}-03$ & $2.82 \mathrm{E}-03$ & 2.92E-03 \\
\hline$E_{\text {geo }}$ & $2.75 \mathrm{E}-03$ & $2.41 \mathrm{E}-03$ & $2.30 \mathrm{E}-03$ & 2.31E-03 \\
\hline
\end{tabular}

value of $\alpha=2$ relatively yields the lowest error, hence, validating our choice of the default value of $\alpha=2$ in the HyMOFLS algorithm.

\section{Coupling with momentum solver}

\subsection{Incompressible Navier-Stokes equations}

In order to describe the incompressible multiphase flow, the following conservative form of the incompressible Navier-Stokes equations are solved

$$
\begin{aligned}
& \boldsymbol{\nabla} \cdot \boldsymbol{u}=0, \\
& \frac{\partial \rho \boldsymbol{u}}{\partial t}+\nabla \cdot(\rho \boldsymbol{u} \otimes \boldsymbol{u})=-\nabla P+\nabla \cdot(2 \mu \boldsymbol{D})+\boldsymbol{B},
\end{aligned}
$$

where $\boldsymbol{u}$ is the velocity field, $\rho$ is density, $\mu$ is dynamic viscosity, $P$ is the pressure field, $\boldsymbol{D}$ is the strain rate tensor given as $\boldsymbol{D}=\frac{1}{2}\left(\boldsymbol{\nabla} \boldsymbol{u}+(\boldsymbol{\nabla} \boldsymbol{u})^{T}\right)$, and $\boldsymbol{B}$ is the sum of the body and surface tension forces. $\boldsymbol{B}=\boldsymbol{B}_{\mathrm{b}}+\boldsymbol{B}_{\mathrm{st}}$ where $\boldsymbol{B}_{\mathrm{b}}$ is the force due to body gravity and $\boldsymbol{B}_{\mathrm{st}}$ is the force due to surface tension which is given as $\boldsymbol{B}_{\mathrm{st}}=\sigma \kappa \delta_{I} \boldsymbol{n} . \sigma$ represent the surface tension, $\boldsymbol{n}$ is the liquid/gas interface unit normal, $\kappa$ is the curvature of the interface, and $\delta_{I}$ is the Dirac delta function centered on surface of the interface. In this work, we neglect force due to gravity unless explicitly specified. The mass conservation is ensured through the solution of Equation (8).

In the context of multiphase flows, an interface $\Gamma$ separates the liquid from the gaseous phase. The material properties such as density and viscosity are constant within each phase, i.e., $\rho=\rho_{\text {liq }}$ and $\mu=\mu_{\text {liq }}$ in liquid phase and $\rho=\rho_{\text {gas }}$ and $\mu=\mu_{\text {gas }}$ in gaseous phase. But these properties are subject to a jump at the interface; the jump condition can be written as $[\rho]_{\Gamma}=\rho_{\text {liq }}-\rho_{\text {gas }}$ and $[\mu]_{\Gamma}=\mu_{\text {liq }}-\mu_{\text {gas }}$. The velocity field remain continuous across the interface, hence $[\boldsymbol{u}]_{\Gamma}=0$. However, the pressure is not continuous across the interface and it is possible to write the pressure jump [18] across an inert interface as

$$
[P]_{\Gamma}=\sigma \kappa(\phi)+2[\mu]_{\Gamma}(\nabla \boldsymbol{u} \cdot \boldsymbol{n}) \cdot \boldsymbol{n} .
$$

\subsection{Flow solver}

The flow solver used in this work is ARCHER [48] whose capabilities have been shown in the past works 45, 63, 64, 65. This solver is structured, parallel, and developed for direct numerical simulations (DNS) of complex and turbulent multiphase flows with the application to study primary breakup of liquid fuel jet. The interface between the phases is represented through level set (see Section 2.1.1) and the mass conservation is ensured using volume fraction (see Section 2.1.2). It has been validated for various cases of complex turbulent flow configurations [53, [54, thus, the numerical methods employed in this solver are tailored for treating turbulence in the system. A staggered grid configuration is used with central finite difference scheme for least numerical dissipation. The scalar variables such as liquid volume fraction, density, viscosity, level set function, and pressure are stored in the cell center while the vector variables such as components of velocity and vorticity are stored in cell faces. A consistent mass and momentum flux computation [48] technique is employed in the solver that facilitates to perform simulations of large density ratio between liquid and gas phases. 


\subsubsection{Numerical method}

A second-order central difference scheme is employed for discretization of the spatial derivatives to avoid any dissipation. However, the convection term is discretized using fifth-order WENO scheme to ensure a robust behavior of the solution. The ghost fluid method (GFM) 4 is employed for the spatial discretization of the Poisson equation (Equation (51)) to take into account the force due to surface tension as a pressure jump. The resulting linear system of symmetric and positive definite matrix with five diagonals is solved using multigrid algorithm for preconditioning a conjugate gradient (CG) method [66. The curvature of the interface $\kappa$ (c.f. Equation (2) ) is computed using finite difference approximation of the level set function $\phi$ with the interpolation to the cell-interface crossing point. The atomization process is turbulent and hence, the turbulent inflow condition is generated in the simulations using the synthetic turbulence method 67.

The viscous term is discretized using a formulation proposed by Sussman et al. 68. This method achieves second-order accuracy in regions away from the liquid/gas interface while first-order accuracy near the interface. The time integration of the Navier-Stokes equations is based on a predictor-corrector scheme with the time step size $\Delta t$ determined based on a CFL condition similar to that of Kang et al. 69. For a value of $\mathrm{CFL}=\gamma$, the time steps size is computed by satisfying the inequality

$$
\Delta t \leq \frac{\gamma}{\left(\frac{\left(C_{\mathrm{CFL}}+V_{\mathrm{CFL}}\right) \sqrt{\left(C_{\mathrm{CFL}}+V_{\mathrm{CFL}}\right)^{2}+4\left(G_{\mathrm{CFL}}\right)^{2}+4\left(S_{\mathrm{CFL}}\right)^{2}}}{2}\right)}
$$

where $C_{\mathrm{CFL}}, V_{\mathrm{CFL}}, G_{\mathrm{CFL}}$, and $S_{\mathrm{CFL}}$ represent the CFL conditions based on convective, viscous, gravity, and surface tension (capillary) forces, respectively. Now, considering the components of velocity $\boldsymbol{u}=[u, v, w]^{T}$ and acceleration due to gravity $\boldsymbol{g}=\left[g_{x}, g_{y}, g_{z}\right]^{T}$, each CFL number is computed as

$$
\begin{gathered}
C_{\mathrm{CFL}}=\frac{\max (|u|)}{\Delta x}+\frac{\max (|v|)}{\Delta y}+\frac{\max (|w|)}{\Delta z}, \\
V_{\mathrm{CFL}}=\max \left(\frac{\mu_{\mathrm{liq}}}{\rho_{\mathrm{liq}}}, \frac{\mu_{\mathrm{gas}}}{\rho_{\mathrm{gas}}}\right) \times\left(\frac{2}{(\Delta x)^{2}}+\frac{2}{(\Delta y)^{2}}+\frac{2}{(\Delta z)^{2}}\right), \\
G_{\mathrm{CFL}}=\sqrt{\frac{\left|g_{x}\right|}{\Delta x}}+\sqrt{\frac{\left|g_{y}\right|}{\Delta y}}+\sqrt{\frac{\left|g_{z}\right|}{\Delta z}}, \text { and } \\
S_{\mathrm{CFL}}=\sqrt{\frac{\sigma \max (|\kappa|)}{\rho_{\mathrm{gas}}(\Delta x)^{2}}}
\end{gathered}
$$

where $\max (\cdot)$ is the maximum value determined over all the cells in the domain.

\subsubsection{Projection method}

The momentum conservation equation (see Equation (43p) is solved using a projection method using the procedure of Vaudor et al. 48] with the predictor-corrector time integration scheme. The algorithm of implementation of this projection method in ARCHER is given in Algorithm 6 .

\section{Numerical validation tests}

Several two-phase flows validation tests are now presented to assess the behavior of the HyMOFLS framework of liquid/gas interface reconstruction. First, a two-phase double shear layer under extreme convective conditions is presented followed by the Rayleigh-Taylor instability, binary droplet collision, and finally, the results from Rayleigh-Plateau instability are presented and compared against the experimental observations [70]. 
Algorithm 6 Projection method in ARCHER solver.

1: Compute $\boldsymbol{u}^{*}$ (Predictor step):

$$
\boldsymbol{u}^{*}=\frac{1}{\rho^{n+1}}\left(\rho^{n} \boldsymbol{u}^{n}-\frac{\Delta t}{V}\left(\nabla \cdot\left(\boldsymbol{u}^{n} \otimes\left(\rho^{n} \boldsymbol{u}^{n}\right)\right)+\nabla \cdot\left(2 \mu^{n} \boldsymbol{D}^{n}\right)+\boldsymbol{B}\right)\right)
$$

where $V=\Delta x \times \Delta y \times \Delta z$ is the total volume of a computational cell.

2: Solve for pressure $P^{n+1}$ (Poisson equation for pressure):

$$
\boldsymbol{\nabla} \cdot\left(\frac{1}{\rho^{n+1}}\left(\nabla P^{n+1}\right)\right)=\frac{\boldsymbol{\nabla} \cdot \boldsymbol{u}^{*}}{\Delta t}
$$

3: Compute $\boldsymbol{u}^{n+1}$ (Corrector step):

$$
\boldsymbol{u}^{n+1}=\boldsymbol{u}^{*}+\frac{\Delta t}{\rho^{n+1}}\left(-\nabla P^{n+1}\right)
$$

\subsection{Two-phase double shear layer}

The two-phase double shear layer destabilization under highly convective regime is an empirical test used in our flow solver ARCHER to assess the stability of the numerical simulation when an interface reconstruction method is employed. In this test, a low speed liquid layer is destabilized by the high speed gas flowing above and below it under highly convective conditions of infinite Reynolds number (i.e., $R e=$ $\infty, \nu=0$ ) and infinite Weber number (We $=\infty, \sigma=0$ ). Under such conditions, the chances that NavierStokes equation becomes unstable are very high especially when the liquid/gas interface reconstruction is not accurate. This is because the inaccurate interface reconstruction can lead to inaccuracy in density computation and this error is propagated to the velocity components through Navier-Stokes equations thereby destabilizing the entire system. This test specifically assess the accuracy of interface reconstruction under extreme environment thereby giving the true capability of the HyMOFLS method. Furthermore, numerical simulations of liquid jet/sheet atomization processes involve complex topological structures arising under various flow conditions that need to be well captured.

To perform this test, we consider an $L \times L$ double shear layer configuration as shown in Figure 26 with $L=0.003$ being the length and width of the $2 \mathrm{D}$ domain with $\delta=L / 10$ being the width of the liquid layer. The density ratio between liquid and gas is taken to be 1000 . The viscous and surface tension forces are assumed to be many orders of magnitude smaller than the convective term, and therefore, are neglected. Thus, the flow Reynolds number $\mathrm{Re}=\infty$ and liquid based Weber number $\mathrm{We}=\infty$. A divergence free initial velocity field is prescribed in the domain given as

$$
\begin{aligned}
& u=A-0.04 \cos \left(\frac{2 \pi x}{L}\right)\left(\frac{L}{x}\right)\left(\frac{-2}{\delta}\right) \exp \left(-\frac{2 y}{\delta}\right), \\
& v=0.04 \sin \left(\frac{2 \pi x}{L}\right) \exp \left(-\frac{2 y}{\delta}\right),
\end{aligned}
$$

in which the value of $A$ is taken as

$$
A=\left\{\begin{array}{l}
30, \text { in gas phase } \\
2, \text { in liquid phase }
\end{array}\right.
$$

Five mesh resolutions are considered in this test case ranging from $32^{2}$ to $512^{2}$ with doubly periodic boundary conditions. The simulations are performed until the physical time $t=3 \times 10^{-3}$. The objective in this test is to maintain the total kinetic energy and maximum velocity devoid of intermittent bursts anywhere in the domain. The results shown for this test pertain to those obtained on a $128^{2}$ mesh resolution. 


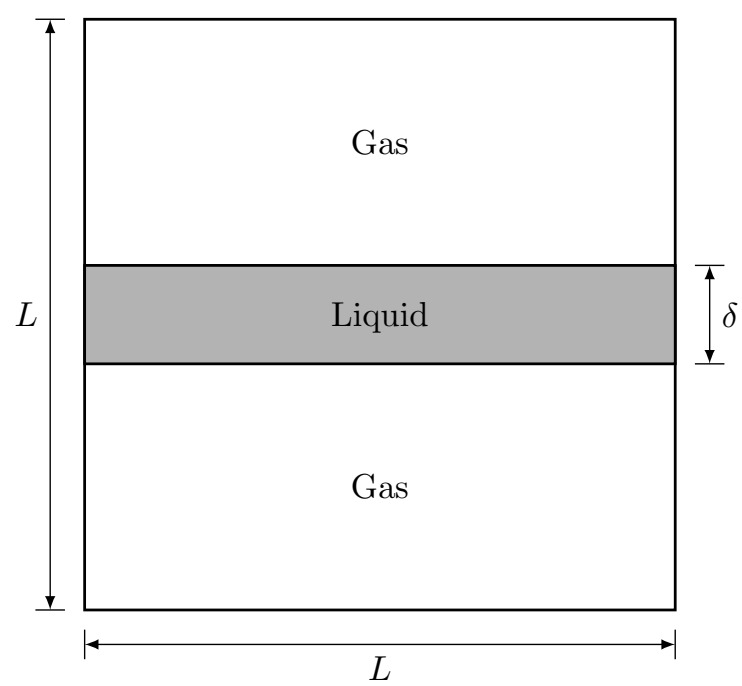

Figure 26: Configuration of a 2D double shear layer.

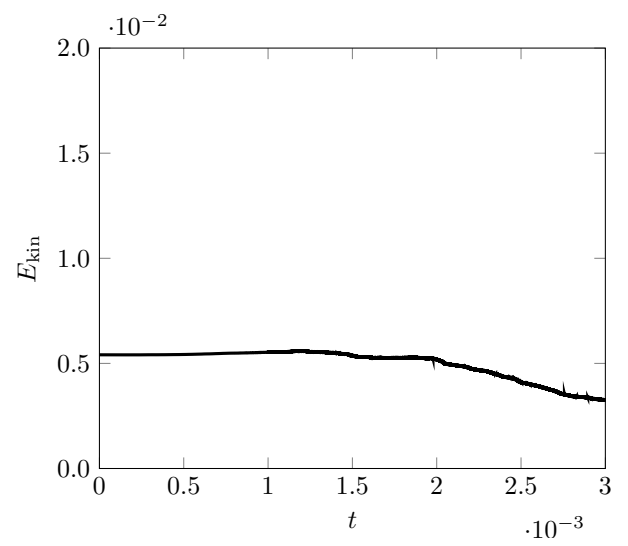

(a) Total kinetic energy.

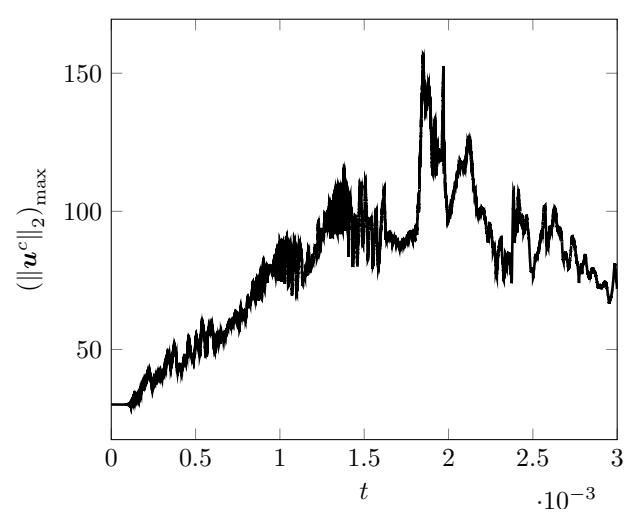

(b) Maximum velocity magnitude.

Figure 27: Time evolution of total kinetic energy and maximum cell centered velocity magnitude for $128^{2}$ mesh resolution.

First, the plots of the time evolution of the total kinetic energy (sum of kinetic energies of liquid and gas phase) and maximum cell centered velocity magnitude in the computational domain are shown in Figure 27. The cell centered velocity $\left\|\boldsymbol{u}^{c}\right\|_{2}$ is computed as $\left\|\boldsymbol{u}^{c}\right\|_{2}=\sqrt{\left(u^{c}\right)^{2}+\left(v^{c}\right)^{2}}$ and the maximum cell centered velocity is computed according to the expression $\left(\left\|\boldsymbol{u}^{c}\right\|_{2}\right)_{\max }=\max _{\forall \text { domain }}\left\|\boldsymbol{u}^{c}\right\|_{2}$. Due to the periodic boundary conditions, the total kinetic energy $E_{\text {kin }}$ must remain constant over time. From Figure 27a, it can be observed that the kinetic energy remains almost constant over all times with a reduction in value observed after $t=2 \times 10^{-3}$. This reduction could be attributed to the artificial diffusion introduced by the numerical discretization schemes used in the solver.

Next, focusing on Figure 27b that shows the time evolution of the maximum cell centered velocity magnitude, there are no intermittent velocity bursts observed. This highlights the ability of the HyMOFLS method to reconstruct the liquid/gas interface accurately. As shown by Asuri Mukundan et al. [45, the non-burst of the velocity goes to show that the interface reconstruction method is accurate for capturing especially the under-resolved liquid structures even under extreme convective flow conditions.

Finally, the phase interface of the destabilized shear layer for multiple time instants using the HyMOFLS method is shown in Figure 28 obtained on $128^{2}$ mesh resolution. It can be seen that there are few pockets 
in the computational domain away from the interface that are having higher velocity than those near the interface. It is to be remarked that the employed mesh resolution well resolves the interface resulting in its reconstruction using CLSVOF method indicated by blue color (see Section 2.3.).

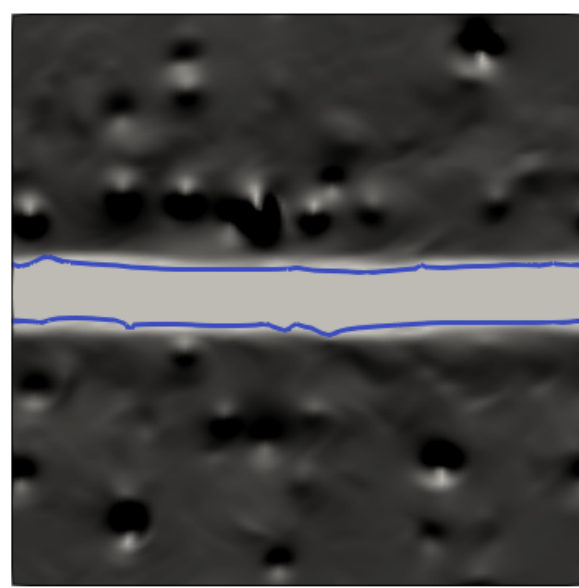

Velocity Magnitude

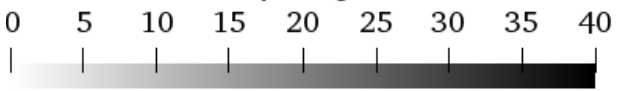

(a) $t=1.07 \mathrm{~ms}$

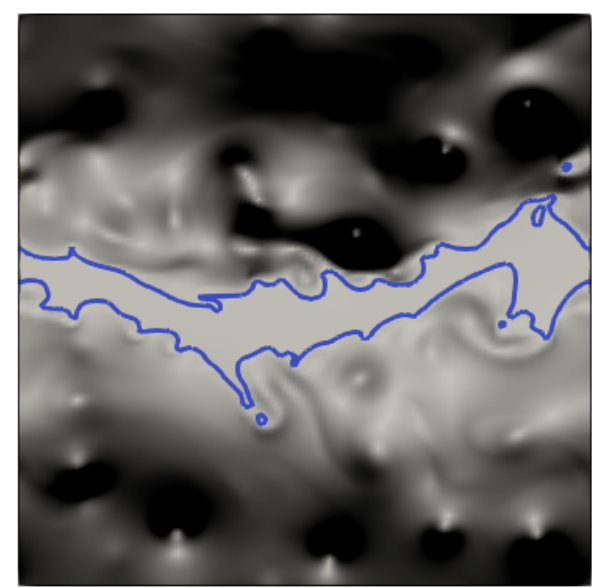

Velocity Magnitude

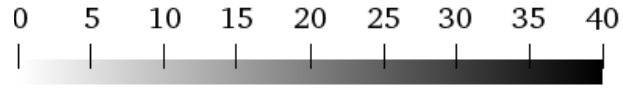

(b) $t=2.00 \mathrm{~ms}$

Figure 28: Phase interface (solid line) colored by interface method label and contour of magnitude of velocity for two-phase double shear layer test using HyMOFLS method for $128^{2}$ mesh resolution. Blue color of interface indicate CLSVOF interface reconstruction chosen within the context of HyMOFLS method.

\subsection{Rayleigh-Taylor instability}

Next, we employ the HyMOFLS method to simulate the the growth of a Rayleigh-Taylor instability. An extensive number of works in the past have focused on studying and analyzing this instability, for example, Refs. 71, 72, 73. Although these tests were performed devoid of surface tension effect, the recent works 19, 20, 21 have presented the results that includes this effect for the realistic simulation of the growth of the instability. In this test, the initial perturbed interface between the heavier (more dense) liquid on top and lighter (less dense) liquid on the bottom is allowed to grow exponentially over time. Within the context of this work, we follow the configuration as described by Desjardins and Pitsch [21]. In order to study the mesh convergence, we consider five mesh resolutions ranging from $32 \times 128$ to $512 \times 2048$ to discretize the computational domain. The mesh convergence study is performed for an error metric of depth of penetration of the mushroom head.

The initial interface given by the zero-level of the iso-contour of the level set function $\phi$ (within the context of ARCHER solver) is expressed as

$$
\phi(x, y)=y+A \cos (2 \pi x)
$$

where $A=0.05$ is chosen for this test case. This initial interface is placed inside a $[0,1] \times[0,4]$ computational domain with the periodic boundary condition along the horizontal direction while wall boundary condition along the vertical direction. The densities of the top fluid and bottom fluid (denoted as fluid 1 and fluid 2 , respectively) are $\rho_{1}=1.225 \mathrm{~kg} / \mathrm{m}^{3}$ and $\rho_{2}=0.1694 \mathrm{~kg} / \mathrm{m}^{3}$, respectively. The dynamic viscosity of the two fluids are taken as $\mu_{1}=\mu_{2}=3.13 \times 10^{-3} \mathrm{~kg} / \mathrm{ms}$ with the surface tension coefficient being $\sigma=0.1337 \mathrm{~kg} / \mathrm{s}^{2}$, and the acceleration due to gravity is $g=9.81 \mathrm{~m} / \mathrm{s}^{2}$. This test is run upto a physical time of $t=1.2 \mathrm{~s}$. 
Figure 29 shows the time evolution of the phase interface for the finest mesh resolution $512 \times 2048$ colored using the URLS/RLS tagging algorithm. The blue color of the interface indicate that it is reconstructed using CLSVOF method within the context of HyMOFLS method. Since the mesh resolution $512 \times 2048$ is well resolving the interfacial regions, the URLS/RLS tagging algorithm of HyMOFLS method is determining all the regions of the interface to be well resolved and hence tagging it with CLSVOF method of interface reconstruction. It is to be remarked that these results agree well with the study by Desjardins and Pitsch 21] (c.f. Figures 22 and 23 in this reference).

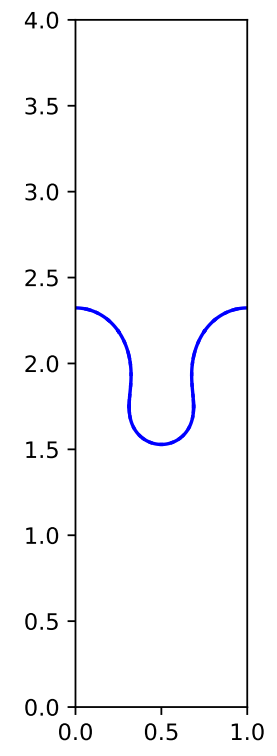

(a) $t=0.7 \mathrm{~s}$

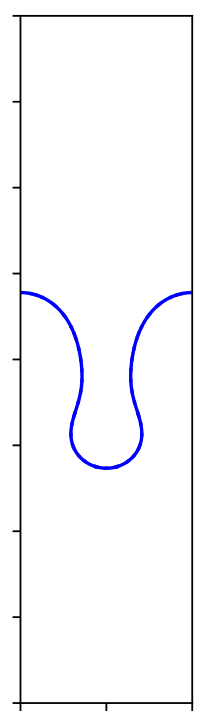

(b) $t=0.8 \mathrm{~s}$

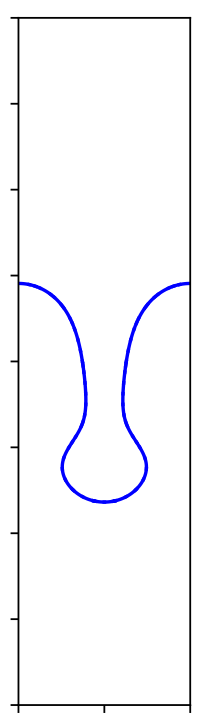

(c) $t=0.9 \mathrm{~s}$

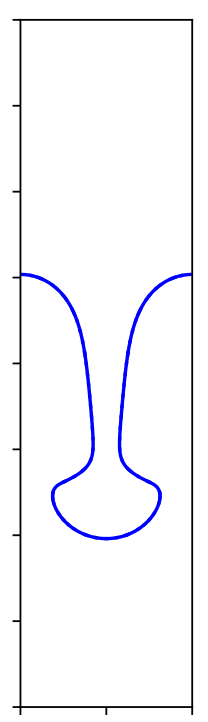

(d) $t=1.0 \mathrm{~s}$

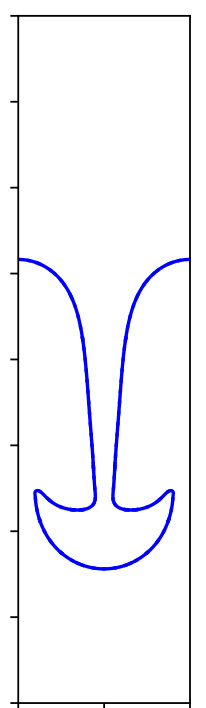

(e) $t=1.1 \mathrm{~s}$

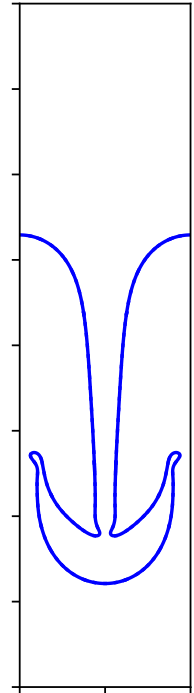

(f) $t=1.2 \mathrm{~s}$

Figure 29: Time evolution of phase interface for Rayleigh-Taylor instability test using HyMOFLS method with $512 \times 2048$ mesh resolution. Blue color of interface indicate CLSVOF tagged regions within the context of HyMOFLS method.

Due to the growing instability, the denser liquid (top liquid) accelerates and pushes its way through the lighter liquid (bottom liquid) forming a mushroom head-like structure hereon called as spike. The depth (in vertical $y$-direction) until which the denser fluid penetrates into the lighter fluid is defined as the spike penetration within this work. An error in this spike penetration for each mesh resolution is determined as the difference between the spike penetration of a given mesh resolution with respect to the reference solution computed on $512 \times 2048$ grid. To that end, the plots of the phase interface for various mesh resolution overlapped on each other for $t=1.0,1.1$, and 1.2 are shown in Figure 30 with the arrows indicating the direction of increasing mesh resolutions. It can be clearly seen that there are overlap among the phase interfaces for different mesh resolutions.

Next, we show the plot of mesh convergence of this spike penetration error at different time instants along with the first-order and second-order error convergence lines in Figure 31. We can observe that the error converges along a second-order slope which indicates that higher the mesh resolution lower is the spike penetration error validating our observation on overlap in phase interface (c.f. Figure 30.

\subsection{Binary droplet head-on collision}

So far, we have assessed the capability and accuracy of HyMOFLS method coupled with ARCHER solver for $2 \mathrm{D}$ cases. We now test it for the binary droplet head-on collision in which two individual droplets collide head-on such that their centers are oriented along the same axis. Such droplet collision scenarios are physically relevant and realistically possible scenario in a typical atomization simulation. Once the two droplets collide, there are three outcomes - droplet coalescence, bounce back with, and without satellite 


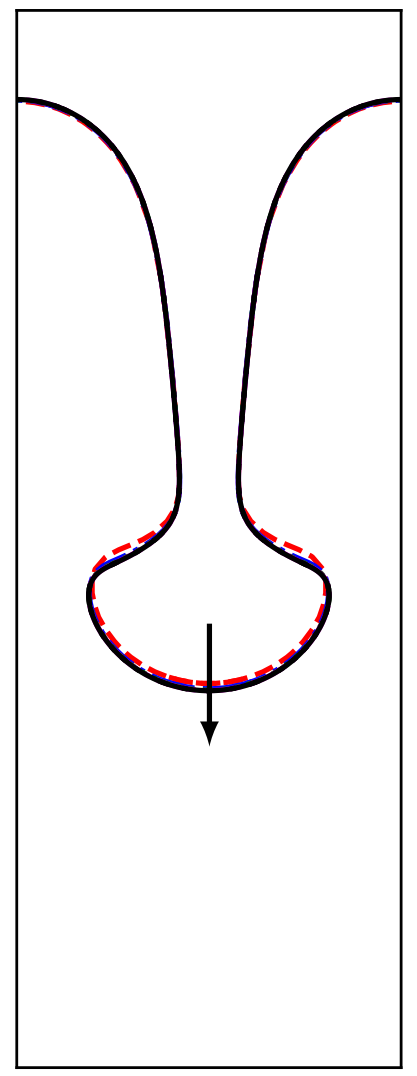

(a) $t=1.0 \mathrm{~s}$

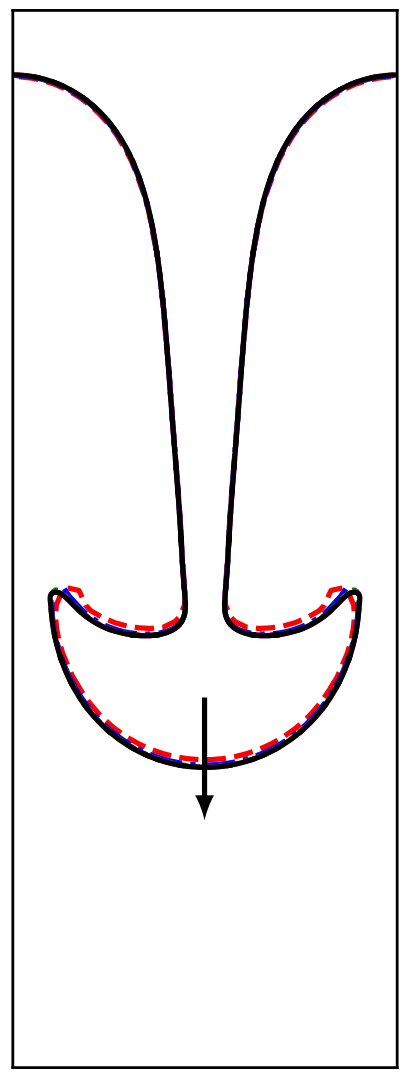

(b) $t=1.1 \mathrm{~s}$

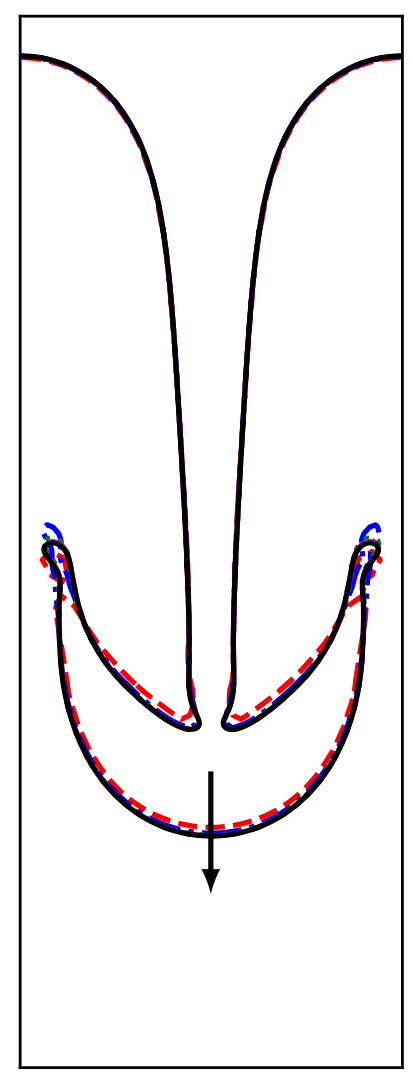

(c) $t=1.2 \mathrm{~s}$

Figure 30: Overlap of phase interfaces for various mesh resolutions for Rayleigh-Taylor instability using HyMOFLS method. Arrow indicates increasing mesh resolutions from $32 \times 128[--), 64 \times 256[-\cdots, 128 \times 512[\cdots \cdots, 256 \times 1024[-\cdots$, to $512 \times 2048$

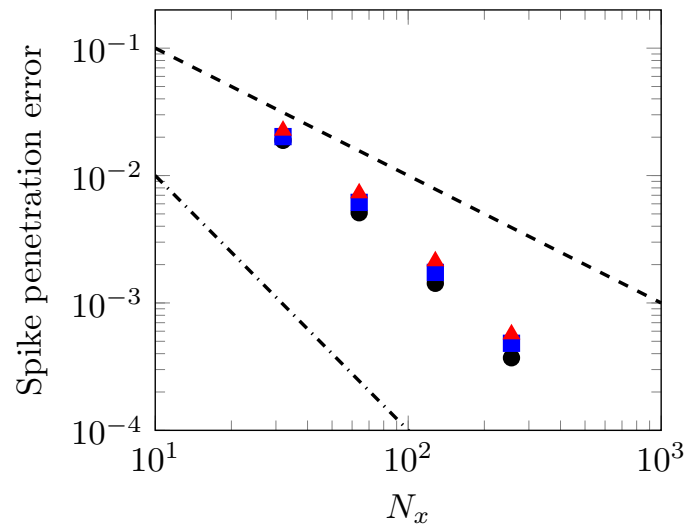

Figure 31: Mesh convergence of spike penetration error for Rayleigh-Taylor instability test at $t=1.0 \mathrm{~s}$ $\Lambda$ shown along with first-order convergence --- and second-order convergence $[----)$ lines.

droplet. The aim of this test is to compare the results from the simulations using HyMOFLS method and experiments [70] in a qualitative fashion. To that end, we have chosen the configuration, initial, and boundary conditions for this test as described by Tanguy and Berlemont 66. Two identical droplets of 
diameter $D=800 \mu \mathrm{m}$ are placed inside a $6 D \times 12 D \times 6 D\left(L_{x} \times L_{y} \times L_{z}\right)$ domain with their respective centers located at $\left(0, L_{y} / 9,0\right)$ and $\left(0,-L_{y} / 9,0\right)$ with Weber number We $=23$. These droplets are made to collide head-on with relative velocity of $1.44 \mathrm{~m} / \mathrm{s}$ and an impact parameter of 0 . The computational domain with slip wall boundary conditions on all its faces is discretized using a $128 \times 256 \times 128$ mesh resulting in equidistant mesh spacing of $\Delta x=\Delta y=\Delta z=37.5 \mu \mathrm{m}$ leading to $D / \Delta x=21.33$. The simulation is performed with a CFL $=0.25$. The results from the simulation are compared with the experimental study by Ashgriz and Poo [70] with droplet Weber number We $=23$ and impact parameter of 0.05 which is close to zero.

The comparison of the results between the simulation and experiments is shown in Figure 32 . The simulation results are observed to be agreeing well with the experimental observations along with the reproduction of the morphology of the droplets before, during, and after head-on collision as seen in Figure $32 \mathrm{~b}$. Next, it can be seen from Figure 32c that the hybrid framework is appropriately tagging the interfacial regions with the MOF (red regions) and CLSVOF (blue regions) methods. Overall, it can be inferred that the HyMOFLS method demonstrates the capability and accuracy to simulate droplet collision phenomenon.

\subsection{Rayleigh-Plateau instability}

As a final validation case, we test the HyMOFLS interface reconstruction framework for the simulation of Rayleigh-Plateau instability which is driven mainly by the surface tension forces. Similar to the work of Ménard et al. [18, we chose a $L \times L \times L$ domain with $L=150 \mu \mathrm{m}$. The initial profile for the level set function is given according to the following expression

$$
\phi(x, y, z)=a_{0}-r+A_{0} \cos (2 \pi x / \lambda)
$$

where $A_{0}$ is the amplitude of the initial disturbance set to $10 \%$ of the radius of ligament (i.e., cylinder) with the radius $R=33.4 \mu \mathrm{m}, \lambda$ being the wavelength set to $\lambda=2 L, a_{0}=k \lambda / 2 \pi$, and $r=\sqrt{y^{2}+z^{2}}$ are chosen for this test. The initial condition is chosen in order to have the wavenumber satisfying $k \lambda=0.7$ which correspond to fastest growth rate [74. The instability/disturbance is set along the $x$ direction with the boundary conditions in the computational domain chosen to be slip wall along $x$ direction, slip wall along the $y-$ and $z$ - planes, and outflow along $y+$ and $z+$ planes. The in-house Navier-Stokes solver ARCHER 48 has been used to solve the Navier-Stokes equations in the simulations using the HyMOFLS method of interface reconstruction. The physical properties are chosen to be $\rho_{\text {liq }}=1000 \mathrm{~kg} / \mathrm{m}^{3}, \mu_{\text {liq }}=0.001 \mathrm{Ns} / \mathrm{m}^{2}$, $\rho_{\text {gas }}=1 \mathrm{~kg} / \mathrm{m}^{3}, \mu_{\text {gas }}=1.879 \times 10^{-5} \mathrm{Ns} / \mathrm{m}^{2}$, and $\sigma=0.072 \mathrm{~kg} / \mathrm{s}^{2}$. Due to the symmetry of the shape of ligament under investigation, an eighth of the size of the cylindrical ligament is simulated using ARCHER. The dispersion relation for this Navier-Stokes solver has been already shown in the past [18] to be having satisfactory agreement with the linear theory of Weber [74. Therefore, in this paper, we investigate the convergence of the breakup time for various mesh resolutions. To that end, we have considered the mesh resolutions shown in Table 11 with $\Delta x=\Delta y=\Delta z$ for all the cases. It is to be noted that in this table, $N_{x}$ represents the number of cells along $x$-direction.

Table 11: List of mesh resolutions and number of computational cells per diameter for the Rayleigh-Plateau instability with $L=150 \mu \mathrm{m}$.

\begin{tabular}{ccc}
\hline Domain size & Mesh resolution & $\Delta x(=\Delta y=\Delta z)$ \\
\hline & $32^{3}$ & $4.6875 \mu \mathrm{m}$ \\
$L \times L \times L$ & $64^{3}$ & $2.3438 \mu \mathrm{m}$ \\
& $128^{3}$ & $1.1719 \mu \mathrm{m}$ \\
$256^{3}$ & $0.5859 \mu \mathrm{m}$ \\
\hline
\end{tabular}

The simulation is run for a long time for each mesh resolution to observe the breakup of the liquid ligament into mother and satellite drops. Figure 33 show the series of snapshots pertaining to $128^{3}$ mesh resolution displaying the development and propagation of the perturbation leading to the breakup into 

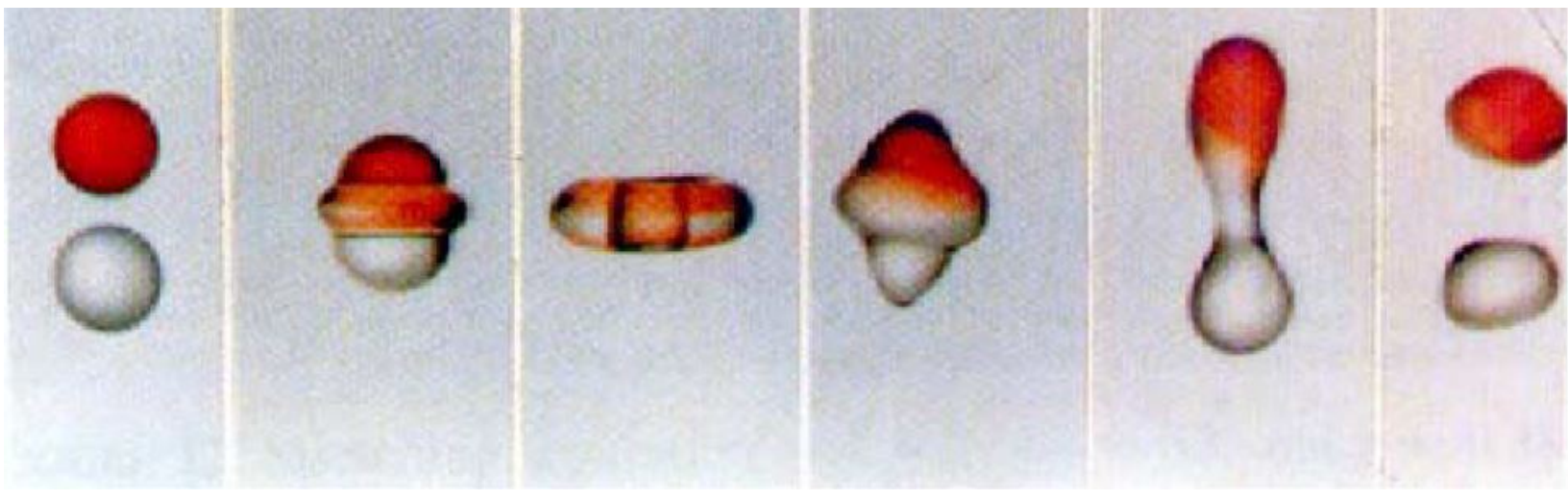

(a) Experimental snapshots from Ashgriz and Poo [70.
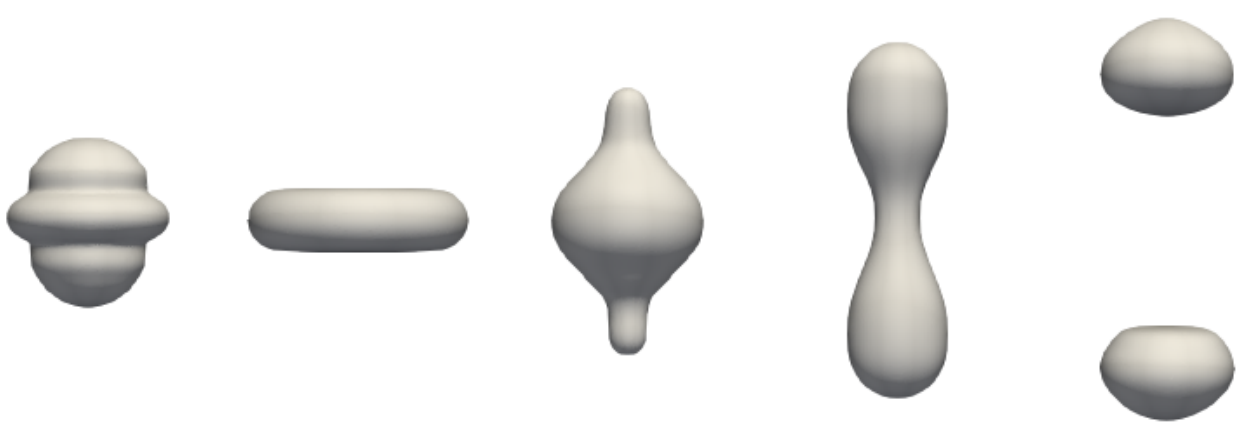

(b) Simulations with HyMOFLS method.
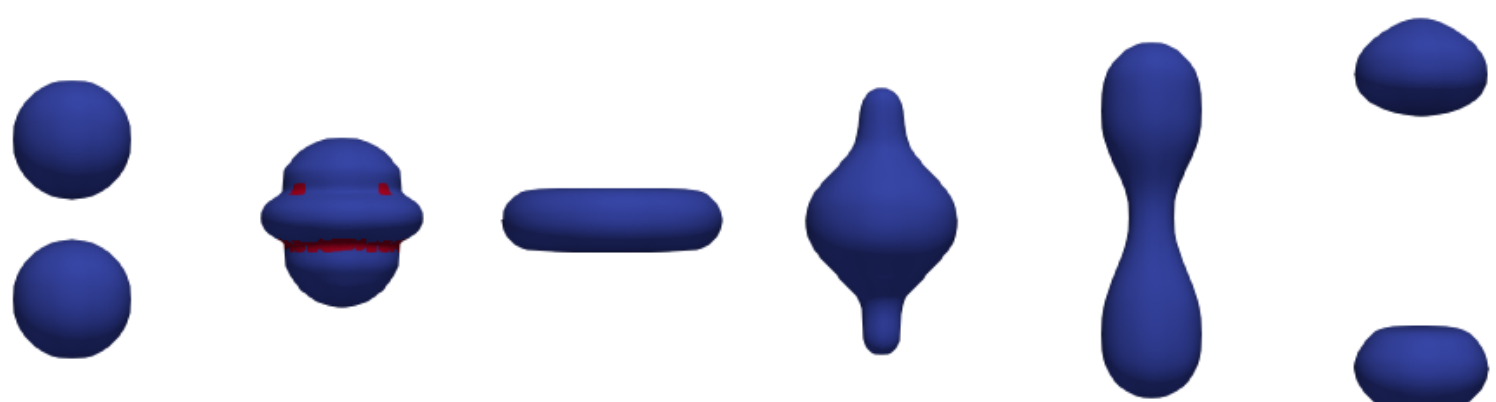

(c) Simulations with HyMOFLS method colored by cell tagging method

Figure 32: Binary droplet collision comparison between experiments 70 and simulations performed using HyMOFLS method with cell tagging colors - red regions represent MOF method tagging and blue region represent CLSVOF method tagging.

mother and satellite drops. The qualitative observation of these instantaneous snapshots show that the results are agreeing with the literature 18,10 .

To quantitatively analyze this test case, the time instance of the breakup is computed. This breakup time is defined as the time instance at which the first breakup event occurs in the simulation. A breakup 
Time: $0.0000 \mathrm{e}+00$

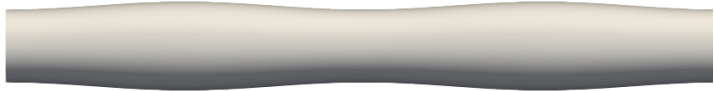

Time: $1.5250 \mathrm{e}-04$

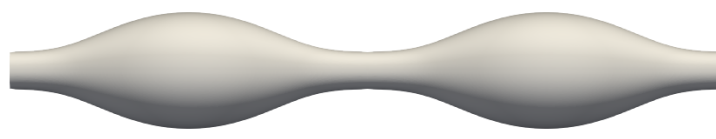

Time: $1.8250 \mathrm{e}-04$

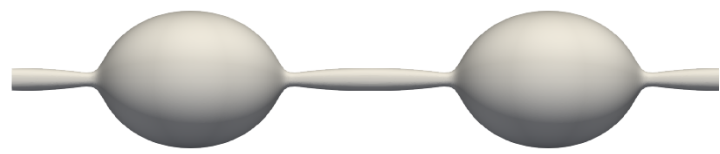

Time: 2.0500e-04
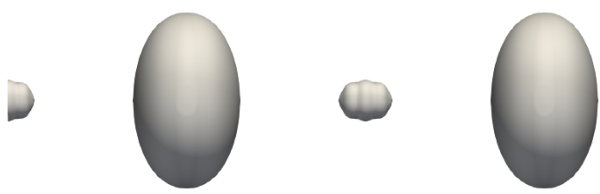

Time: 1.0750e-04

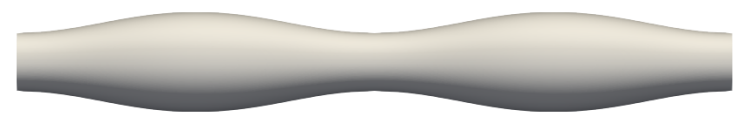

Time: $1.6000 \mathrm{e}-04$

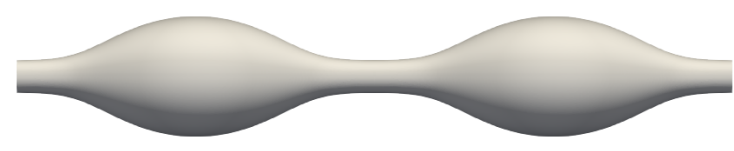

Time: $1.8750 \mathrm{e}-04$

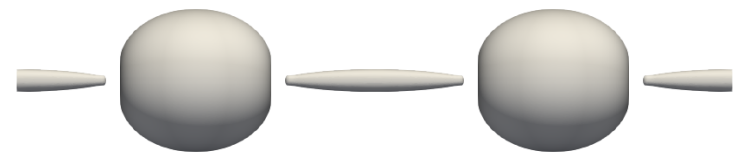

Time: 3.6819e-04

Figure 33: Instantaneous snapshots of the breakup of liquid ligament for the simulation of Rayleigh-Plateau instability using $128^{3}$ mesh resolution.

event within this context is defined as the moment at which more than one liquid structure is formed. At each simulation time step, the number of liquid structures are found using a connected component labelling (CCL) algorithm [75] and the breakup event is characterized by the moment at which there exist more than one liquid structure in the computational domain. The plot of the evolution of the breakup time is shown in Figure 34 for four mesh resolutions ranging from $32^{3}$ to $256^{3}$. From this plot, we can see that the breakup time indeed is converged starting from $N_{x}=64$ for the considered mesh resolutions for the HyMOFLS framework of liquid/gas interface reconstruction.

Finally, we present the CPU time consumption for the HyMOFLS method for various mesh resolutions considered for this test. To that end, we have compartmentalized the total time consumption per iteration into time taken for interface reconstruction subroutines (i.e., HyMOFLS framework subroutines), velocity solver subroutines, and Poisson solver subroutines. The literature works [10, 19] found that the Poisson solver to be the highest time consuming subroutine. Therefore, the results presented in this paper pertain to the relative CPU time consumption for the interface reconstruction subroutines and velocity solver subroutines 


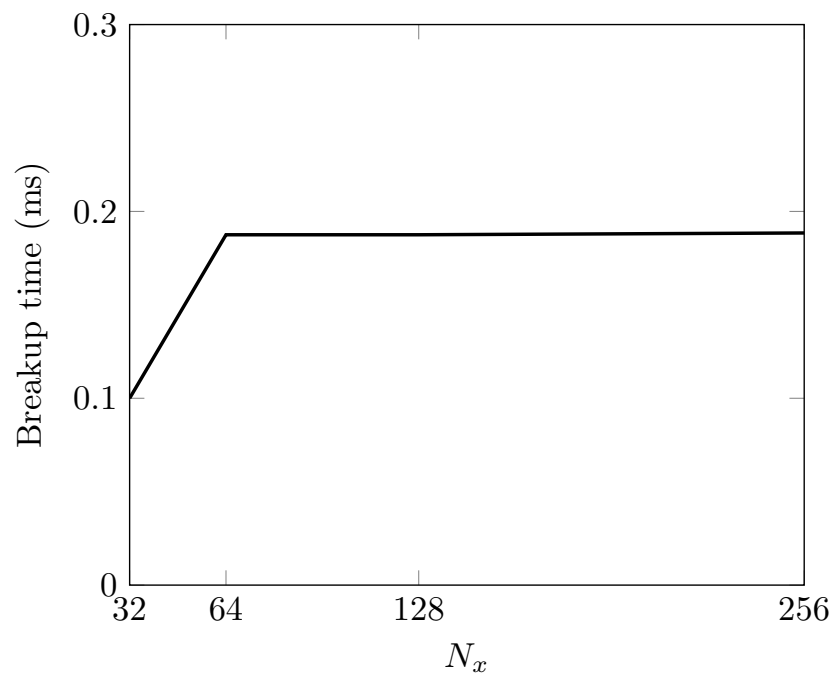

Figure 34: Plot of breakup time for Rayleigh-Plateau instability for various mesh resolutions to observe convergence. $N_{x}$ represents number of cells along $x$-direction.

compared to the Poisson solver. The relative time consumption for interface reconstruction subroutine is computed as the ratio of its time consumption to that of the Poisson solver while the relative time consumption for velocity solver subroutine is computed as the ratio of its time consumption to that of the Poisson solver. Figure 35 show the plots of the time evolution of this quantity for the interface reconstruction subroutine (solid black line) and velocity solver subroutine (dashed red line). It can be seen that for this 3D test case, the time taken for the interface reconstruction subroutines remains predominantly lesser than that of the Poisson solver. The intermittent spike observed around $0.2 \mathrm{~ms}$ in the time evolution of interface reconstruction relative time consumption (solid black line) is attributed to the beginning of tagging of the computational cells with MOF method near and after the breakup event. These intermittent spikes are profoundly visible for the lower mesh resolution while not for the higher mesh resolution. This is due to the fact that with increasing mesh resolution the interface gets well resolved thereby eliminating the need for MOF interface reconstruction within the HyMOFLS method. Based on the presented results, the HyMOFLS framework has demonstrated its capability as a computationally feasible numerical method to simulate liquid breakup.

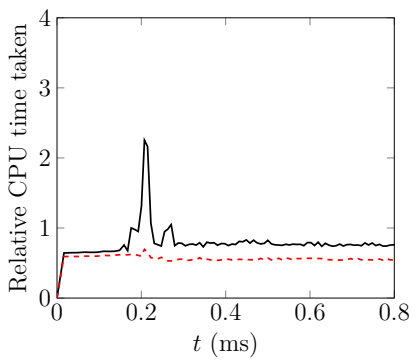

(a) $32^{3}$

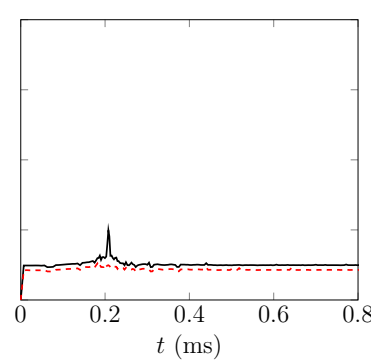

(b) $64^{3}$

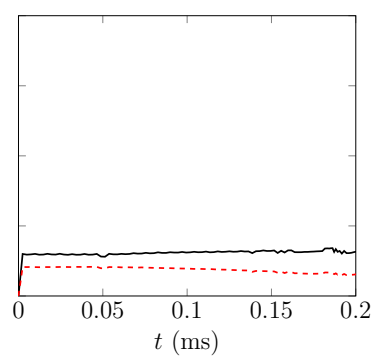

(c) $128^{3}$

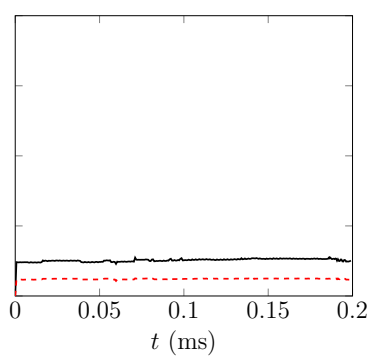

(d) $256^{3}$

Figure 35: Relative CPU time consumption per iteration for interface reconstruction subroutine and velocity solver subroutine - - relative to the time consumption of Poisson solver subroutine for Rayleigh-Plateau instability. 


\section{Engineering applications}

The HyMOFLS method is developed with the objective to accurately and (computationally) efficiently simulate the fuel injection scenarios for real-time engineering applications. So far, the computational comparison tests and validation tests have been performed for simple velocity profiles, flows under high convection, and those that are dominated by surface tension under confined setting and environment. In order to assess the capability of the HyMOFLS method to simulate the flows with fully developed turbulence, we now present two injection simulations - turbulent liquid jet injection into quiescent gas and planar pre-filming Airblast atomization. The simulation conditions for the former are inspired from the case presented by Ménard et al. 18 which is Diesel-like liquid jet injection condition while the latter is inspired from the simulations from the work of Warncke et al. 76]. On one hand, in the case of the Diesel fuel injection in a quiescent gaseous environment, the shear between the liquid and the gas phase and subsequent liquid fuel breakup is caused solely due to the high speed of the liquid Diesel fuel. On the other hand, the liquid fuel sheet on the pre-filmer plate is destabilized due to the high speed gas in the case of Airblast atomization.

\subsection{Turblent liquid jet atomization}

First, we apply the HyMOFLS method to the simulate turbulent liquid jet atomization inspired from the Diesel injection systems. In choosing the operating conditions for this case, we have reduced the Reynolds and Weber numbers compared to the real-time Diesel injection for the sake of computational feasibility of the simulations. The operating conditions for this simulation are given in Table 12

Table 12: Operating condition for the turbulent liquid jet atomization simulation.

\begin{tabular}{cccccccc}
\hline$\rho_{\text {liq }} / \rho_{\text {gas }}$ & $\mu_{\text {liq }} / \mu_{\text {gas }}$ & $\sigma$ & $\mathrm{Re}_{\text {liq }}$ & $\mathrm{We}_{\text {liq }}$ & $\mathrm{Re}_{\tau}$ & $D_{j} / \Delta x$ & $l_{t} / \Delta x$ \\
\hline 27.84 & 120 & 0.06 & 5800 & 11,600 & 58 & 42.74 & 4 \\
\hline
\end{tabular}

A $3 D \times 3 D \times 24 D$ domain is considered where $D=100 \mu \mathrm{m}$ is the diameter of the liquid jet. A uniform Cartesian grid of about 17 million cells is used to discretize the domain resulting in an uniform mesh spacing of $D / \Delta x=42.67$ throughout the domain. Although the smallest liquid structure might not be captured with this employed mesh resolution, no sub-grid scale (SGS) models have been used in the simulations. The liquid fuel is injected at a velocity of $U_{\text {liq }}=100 \mathrm{~m} / \mathrm{s}$. A fully developed turbulent pipe flow velocity profile is imposed on the liquid inlet as the inflow conditions with the velocity fluctuations $u^{\prime}=0.10 \bar{U}_{\text {liq }}$ and the turbulent integral length scale $l_{t}=0.1 D_{j}$. The turbulent inflow boundary conditions are generated using the synthetic turbulence generation method of Klein et al. 67] in which correlated random velocities with a prescribed length scale are generated. We considered this length scale to be equal to $l_{t}$. For the considered mesh resolution, we have $l_{t} / \Delta x \approx 4$ and the turbulent Reynolds number at injection $R e_{\tau}=\rho_{j} u^{\prime} l_{t} / \mu_{j}=58$. The simulation is run upto a time of $t^{*}=t U_{\text {liq }} / D=20$.

Figure 36 show several instantaneous snapshots from the simulations representing the time evolution of the jet penetration in the domain with a time spacing of $\Delta t^{*}=\Delta t U_{\text {liq }} / D=2.5$ between each image. We observe that small ligaments and droplets are being ejected from the mushroom head of the liquid jet due to the Rayleigh-Taylor and Rayleigh-Plateau instabilities. Furthermore, we can observe the three dimensional waves on the surface of the liquid column in the Figure 36 caused by the transverse velocity gradients. These instability waves are vital in the detachment or breakup of the liquid column of the jet into ligaments and droplets.

Upon further analysis of the jet, we find three zones of instability and breakup as shown in Figure 37 . First zone is the near nozzle zone which is upto five jet diameters downstream in which there is no observation of breakup but only generation of waves on the liquid column. In the second zone (or the transition zone), the gas enters the dense part of the liquid jet causing the waves to roll up and leading to first breakup of the liquid ligaments. The third and final zone is chaotic in nature in which many ligaments and drops are ejected from the liquid core thereby leading to primary atomization.

Since we have used HyMOFLS which uses MOF and CLSVOF methods of interface reconstruction, it is interesting to see the regions where these methods are activated/used. To that end, we present snapshots 


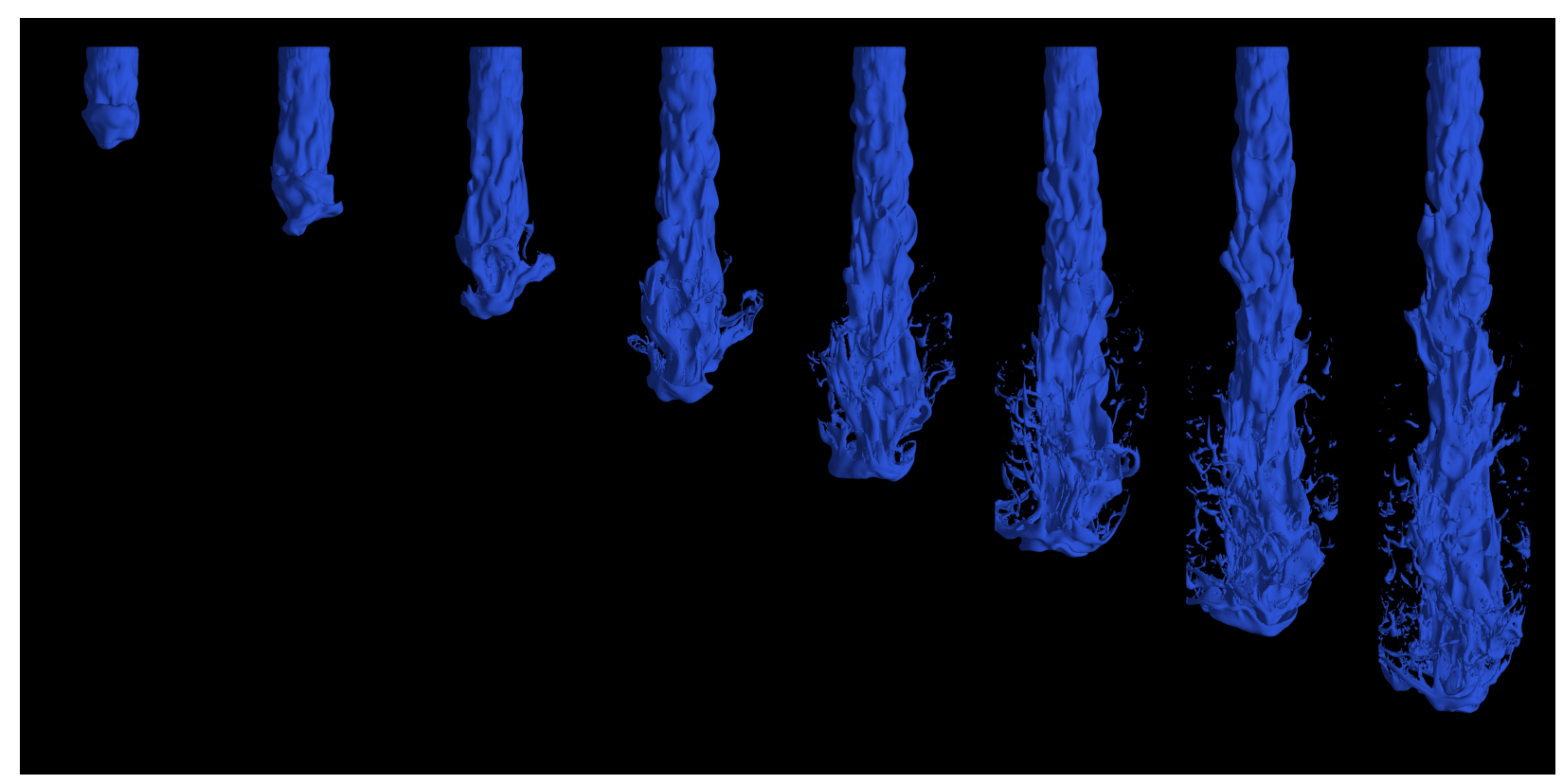

Figure 36: Time evolution of turbulent atomization of liquid diesel jet. The temporal spacing between each consecutive snapshot (from left to right) is $\Delta t^{*}=2.5$.

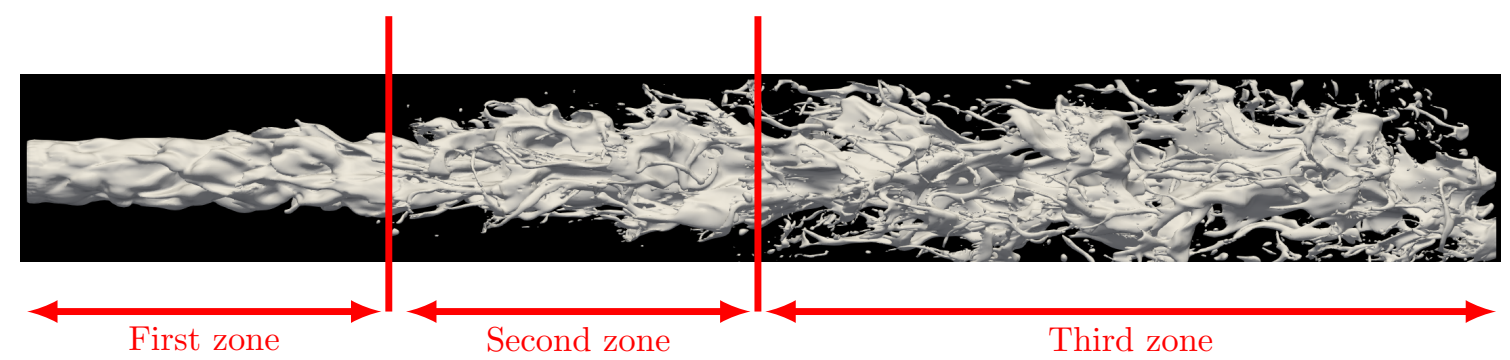

Figure 37: Zones of instabilities on surface of atomizing turbulent liquid jet.

from the simulations of the liquid jet at multiple time instants with each region colored with red (MOF tagged region) and blue (CLSVOF tagged region) in Figure 38. It can be observed that the MOF method is used appropriately for both high curvature regions (waves formed on the liquid column) and the small (under-resolved) liquid structures (ligaments and droplets near the mushroom head breakage). In fact, the initial conditions of this simulation pushes our Navier-Stokes solver towards its limits of operation. Moreover, it can be seen that the results are comparable to those observed in experimental investigation [77] as well as previous numerical studies [18, 10, 19].

On observing Figure 38, we see that as the jet grows downstream, the number of cells tagged with MOF (red cells) are increasing. Therefore, in order to assess the efficiency of the HyMOFLS method with the MOF method, the time evolution of percentage of total number of interfacial cells in the computational domain tagged with MOF method (red regions) and CLSVOF method (blue regions) would be a good metric. To that end, the Figure 39 shows the plot of the time evolution of the percentage of number of MOF and CLSVOF tagged cells for the total simulation duration. It can be seen that when using the HyMOFLS framework, a maximum of $4 \%$ the total number of computational cells containing interface are tagged with MOF method [45]. In our previous study [45, we have demonstrated the ability of the MOF method to simulation primary atomization of turbulent liquid injection, however, yielding higher computational cost. The result presented in Figure 39 demonstrates a significant step-up in terms of computational efficiency for 

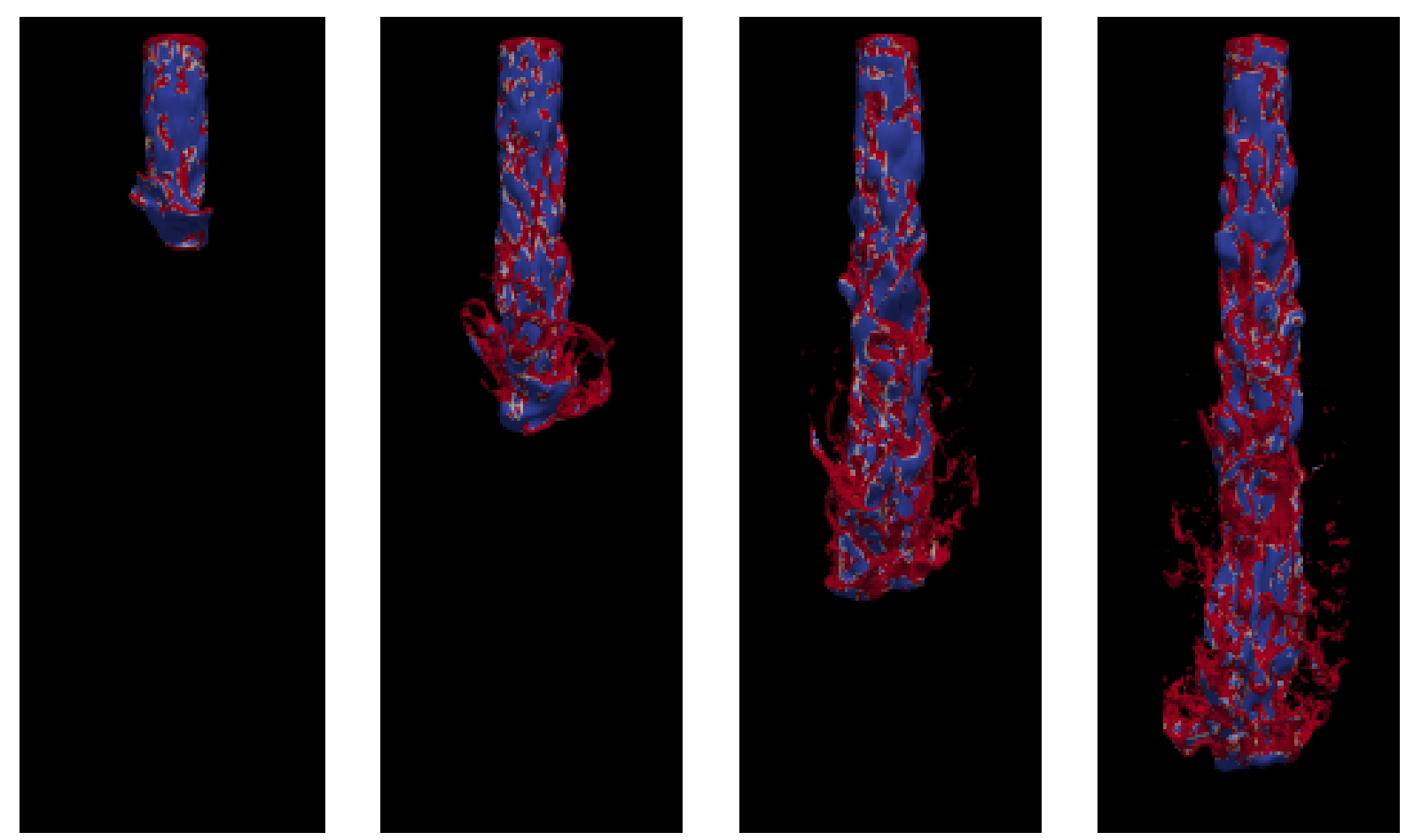

Figure 38: Turbulent atomization of liquid diesel jet with temporal spacing $\Delta t^{*}=3$ between each image. Red regions correspond to MOF interface reconstructed regions and blue regions correspond to CLSVOF interface reconstructed regions.

the HyMOFLS method compared to the MOF method. This justifies the motivation and development of an accurate and robust numerical method for simulating primary atomization at a low to modest computational cost.

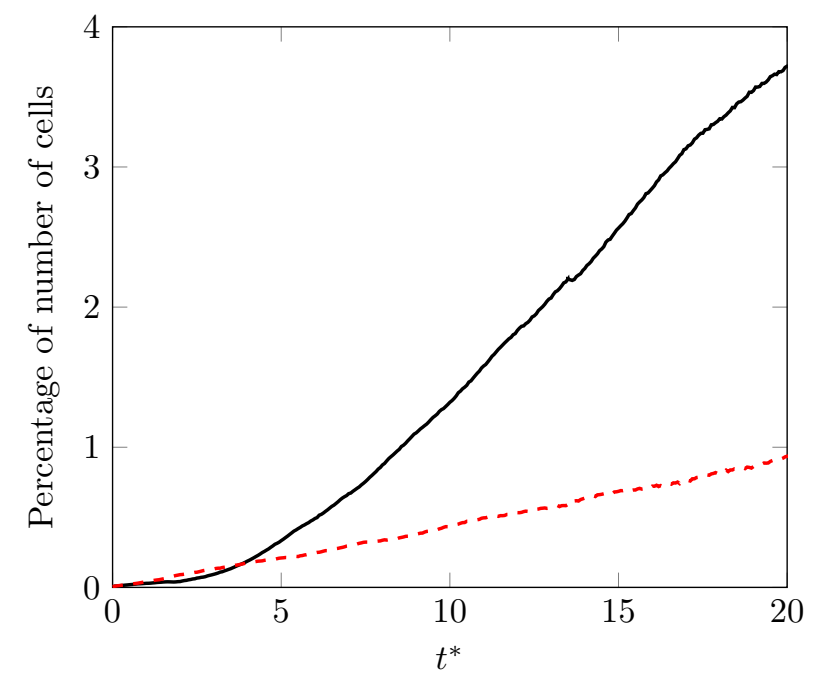

Figure 39: Time evolution of the percentage of number of MOF cells $\square$ and CLSVOF cells --- in the computational domain in the simulation duration. 


\subsection{Airblast atomization}

Finally, we apply the HyMOFLS method to perform simulations of a fuel injection configuration and operating condition relevant for aircraft engine called planar pre-filming Airblast atomization technique. In this technique, a thin film of liquid fuel is injected on a solid plate which is then destabilized by the high speed gas flowing above and below the plate. The simulations of the Airblast atomization presented in this work follows the work of Warncke et al. [76. The computational domain as well as the inlet phase velocity profiles considered in this work are shown in Figures 40 and 41.
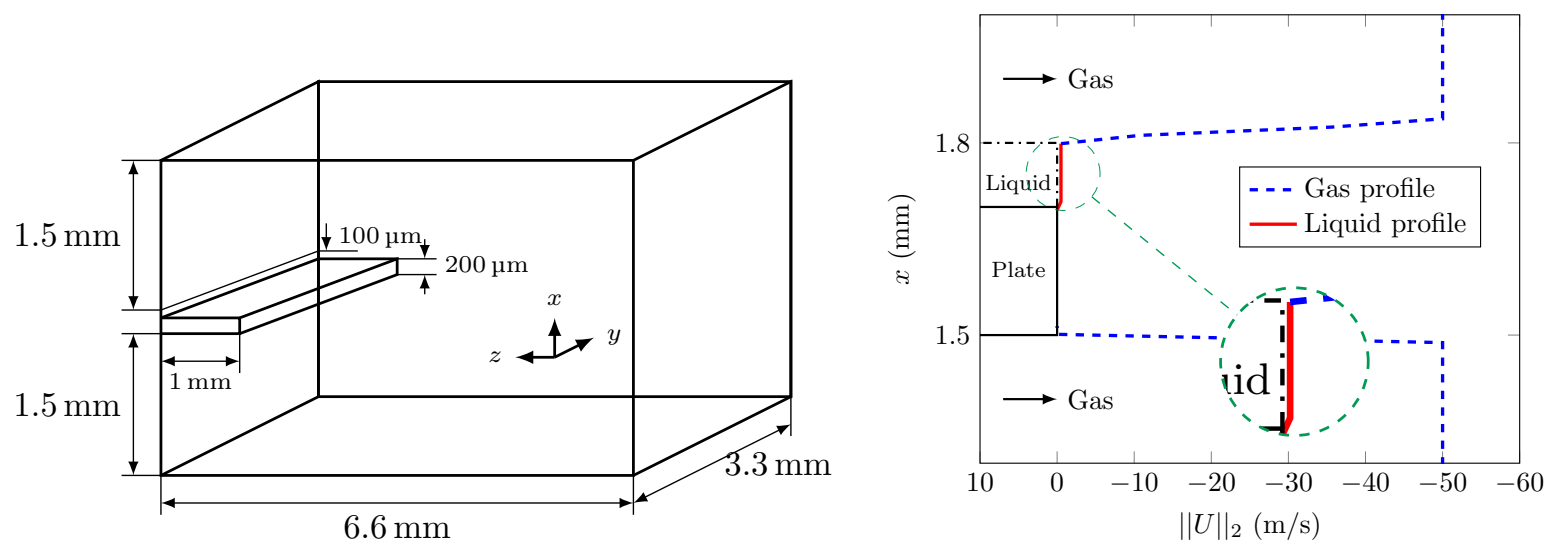

Figure 40: Computational domain.

Figure 41: Inflow velocity profile with zoomed liquid profile view.

This simulation is performed in a $6.6 \mathrm{~mm} \times 3.3 \mathrm{~mm} \times 3.3 \mathrm{~mm}$ domain that is discretized using a $256 \times$ $256 \times 512$ mesh with Shellsol D70 fuel which has similar properties of a Jet A-1 fuel with the liquid velocity $u_{\text {liq }}=0.5 \mathrm{~m} / \mathrm{s}$ and gas velocity $u_{\text {gas }}=50 \mathrm{~m} / \mathrm{s}$. The operating conditions of the simulations corresponding to altitude relight conditions are presented in Table 13. A turbulent flat velocity profile is imposed on the

Table 13: Operating condition for the turbulent liquid jet atomization simulation.

\begin{tabular}{ccccc}
\hline$\rho_{\text {liq }} / \rho_{\text {gas }}$ & $\mu_{\text {liq }} / \mu_{\text {gas }}$ & $\sigma$ & $\mathrm{Re}_{\text {gas }}$ & We $e_{\text {liq.film }}$ \\
\hline 641.67 & 86.83 & 0.0275 & 13,333 & 10.69 \\
\hline
\end{tabular}

liquid and gas phase with their respective velocities as shown in Figure 41 The turbulence is generated only in the gas phase using the synthetic turbulence generation method of Klein et al. 67. with the fluctuations kept to $10 \%$ of the bulk gas velocity and the turbulent integral length scale set to $3 \%$ of the total gas channel height of $8.1 \mathrm{~mm}$. The faces of the solid pre-filmer plate on which the liquid fuel is injected is modeled using staircase immersed boundary method (SIBM).

Figure 42 show the instantaneous snapshots of the top and side views of the atomizing liquid film from the simulation. It can be seen in the top view (Figure 42a) that instability waves are formed on the liquid film on top of the pre-filmer plate. It is speculated that these waves are due Kelvin-Helmholtz instability, however, a more detailed investigation is needed to verify this conjecture. In fact, the simulations accurately captures these waves especially near the edge of the pre-filmer plate when compared to the experimental investigations 78, 76. These waves carry the liquid to the edge of the pre-filmer solid plate thereby forming either a bag which then breakups into droplets or form finger-like ligament structures which then subsequently breakup into droplets due to Rayleigh-Plateau instability. Furthermore, the ligaments and varying sized cluster of droplets produced downstream of the domain are visible in these instantaneous snapshots. Finally, the zoomed portions beside the side view (Figure 42b) with the underlying computational mesh (vertical and horizontal lines in the background) are shown to give insights into the thickness of liquid sheet as well as size of a liquid ligament. It can be seen that for this instantaneous time step, the liquid sheet thickness is 
spanning about $4 \Delta x$ to $7 \Delta x$ while the liquid ligament size varies in the range $7 \Delta x$ to $10 \Delta x$ demonstrating that these liquid structures are well resolved in the simulation.

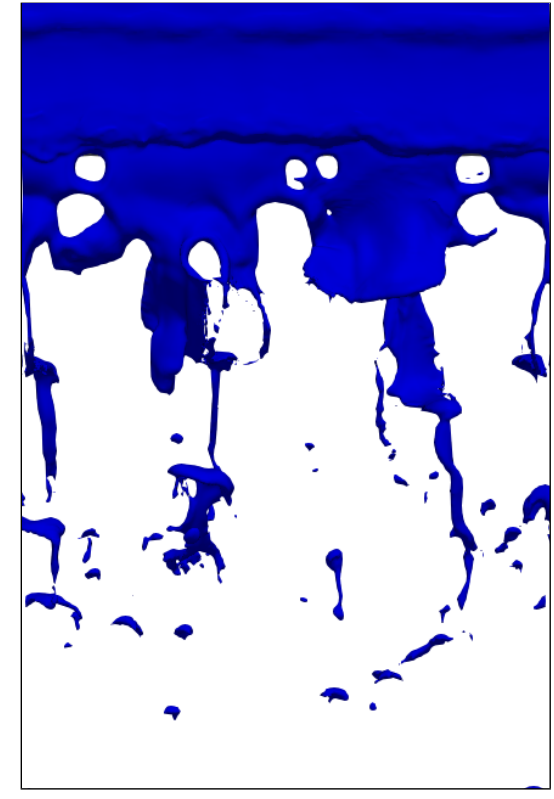

(a) Top view of liquid film.

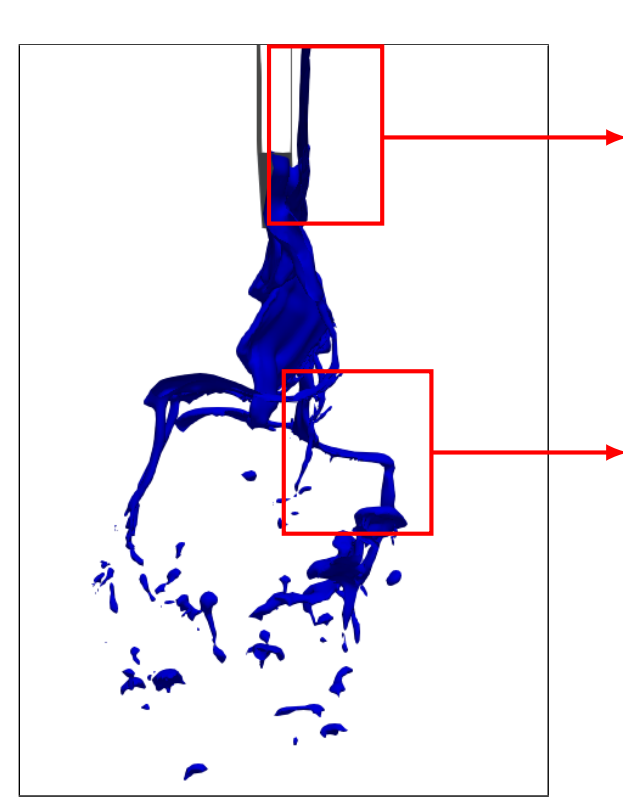

(b) Side view of pre-filmer plate.
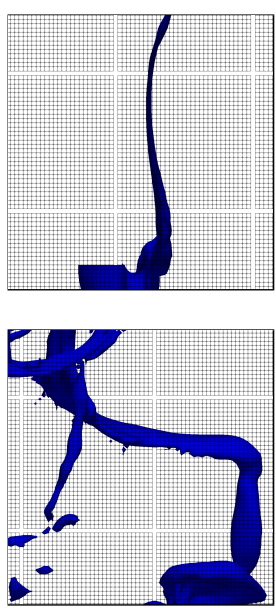

Figure 42. Top and side views of the solid pre-filmer plate in Airblast atomizer. Flow direction is from top to bottom. The zoomed subfigures show the Cartesian grid in the background depicting the computational thickness of the liquid sheet (top subfigure in (b)) and size of a liquid ligament (bottom subfigure in (b)).

Hence, based on these results presented, HyMOFLS method is proved to be a viable interface reconstruction method that captures both resolved and under-resolved liquid structures for an atomization application. Moreover, it was observed to strike a balance between the accuracy and computational cost as well as prevent the artificial/numerically induced premature breakup of the liquid structure from the coherent liquid structure. These are the prominent desirable characteristics for a numerical method especially when used for simulating practically relevant injection configuration and operating conditions.

\section{Conclusions}

A hybrid moment of fluid-level set (HyMOFLS) method has been developed for the purpose of the liquid/gas interface reconstruction within the context of multiphase flows. This hybrid framework involves the usage of an accurate moment of fluid (MOF) method coupled with the computationally cost effective coupled level set volume of fluid (CLSVOF) method for interface reconstruction. The advantage of the HyMOFLS method is that the accurate MOF method has been used only for reconstructing the underresolved liquid structures while the CLSVOF method is used for the resolved liquid structure. Within this method, a narrow band of cells around the interface is tagged (or labeled) with either MOF or CLSVOF method based on the interface resolution quality (IRQ) criterion proposed within the framework of the HyMOFLS method. This criterion relates the interface curvature and mesh spacing to the resolution of the interface thereby identifying the under-resolved regions in the flow. The results from canonical academic verification tests such as Zalesak's notched disk, 2D, and 3D droplet deformation have been presented which compared the performance of the HyMOFLS, MOF, and CLSVOF methods. A parametric study has been performed for the cell tagging (with MOF or CLSVOF) criterion within the framework of HyMOFLS method for these verification tests. 
Then, the HyMOFLS method has been applied to simulate the evolution of the double shear layer under high convective conditions which yielded stable velocity results without any intermittent bursts thereby deeming to be employed for the real-time engineering applications. This method is then assessed to simulate the growth of Rayleigh-Taylor instability for which a second-order spike penetration error was observed. The HyMOFLS method is then put to work to simulate the binary droplet collision wherein the simulation results agreed with the experimental study. As a final validation test, the HyMOFLS method has been employed to simulate the Rayleigh-Plateau instability which is a purely surface tension-driven instability. The qualitative analyses of the results agreed well with the literature. On the quantitative aspect, first, we observed that the breakup time is converged for the mesh resolutions considered and the time taken by the interface reconstruction subroutines are lesser than the Poisson solver which is a typical trend observed in the literature.

Finally, this hybrid framework has been employed to simulate primary atomization of liquid for two configurations - turbulent jet atomization under diesel engine-like condition and planar pre-filming Airblast atomization under aircraft altitude relight operating condition. Despite the complexity of the flow structures, the method is found to be robust and stable in terms of simulating the high speed fuel injection scenarios. In fact, this method is able to capture the surface waves formed on the liquid column for the Diesel jet injection. Furthermore, a good qualitative agreement was found between the simulations and the experiments for the Airblast atomization configuration. For many complex cases of liquid fuel atomization, the HyMOFLS method has been shown to produce accurate results in a computationally cost effective manner thereby making it a viable numerical method for simulations of liquid fuel atomization processes.

\section{Acknowledgements}

The funding for this project from the European Union's Horizon 2020 research and innovation programme under the Marie Skłodowska-Curie grant agreement $\mathrm{N}^{\circ} 675676$ is gratefully acknowledged. The computing time at CRIANN (Centre Régional Informatique et d'Applications Numériques de Normandie) under the scientific project No. 2003008 and at GENCI-[TGCC/CINES/IDRIS] (Grant2019-2613) are also gratefully acknowledged. Anirudh Asuri Mukundan expresses gratitude to Dr. Antoine Lemoine, Dr. Alexandre Poux, Dr. Victor Chéron, and Dr. Alberto Remigi for useful discussions and comments during the MOF method development.

\section{Appendix A. Algorithm of coupled level set volume of fluid (CLSVOF) method}

The CLSVOF method employed in this paper corresponds to that developed by Ménard et al. [18] which was inspired from the work of Sussman and Puckett [17. This method uses both the liquid volume fraction $F$ as well as level set signed distance function $\phi$ for interface reconstruction. In fact, the reconstruction of the interface as well as its geometric properties are both accurate (due to level set function) and volume conservative (due to volume fraction function). From the level set representation, the interface is reconstructed as a plane in $3 \mathrm{D}$ whose expression is given as

$$
\phi_{i, j, k}^{R}=a_{i, j, k}\left(x-x_{i}\right)+b_{i, j, k}\left(y-y_{j}\right)+c_{i, j, k}\left(z-z_{k}\right)+d_{i, j, k},
$$

with $\boldsymbol{n}=[a, b, c]^{T}$ being the normal to the interface and $d$ being the shortest distance of the interface from the computational cell centre.

The list of steps involved in CLSVOF method employed in this work are given in Algorithm 7. As explained in Section 2.1.3, in order to maintain the accuracy and the volume conservation property of the CLSVOF method, mutual correction of level set and volume fraction is implemented which is evident from the lines 1 and 3 in this algorithm. The following paragraphs elucidates the implementation methodology of the steps shown in this algorithm. 


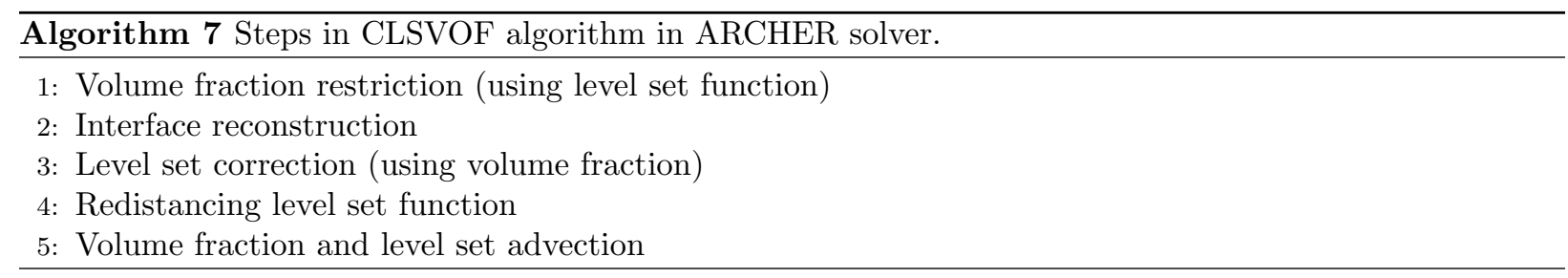

\section{Appendix A.1. Volume fraction restriction}

Quite often, the Eulerian interface reconstruction method such as CLSVOF method can generate jetsam and flotsam which can cause inaccuracies in the conservation of mass in the simulations. Since a directional splitting advection of the quantities is implemented in our in-house ARCHER solver, a natural way to remove these jetsams and flotsams is through volume restriction. We perform this restriction operation as shown in Algorithm 8 .

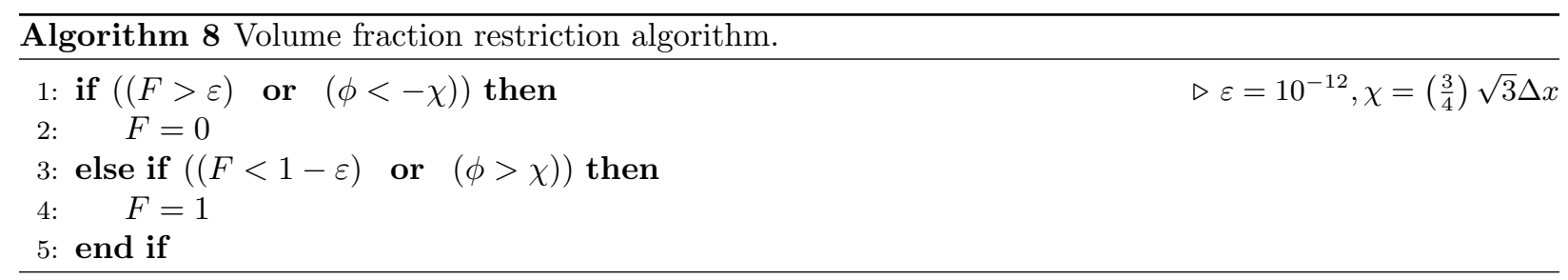

\section{Appendix A.2. Interface reconstruction}

Once the volume fraction is cleaned up, the interface is then reconstructed. The interface reconstruction is performed using the method of minimization of the least square error following the work of Sussman and Puckett [17. The first step in reconstructing the interface is to compute the coefficients $a, b, c$, (coefficients of the unit normal $\boldsymbol{n}$ ) and $d$ which are obtained by minimizing the least square error computed as

$$
\begin{aligned}
E_{i, j, k}^{\mathrm{CLSVOF}}=\int_{z_{k-1 / 2}}^{z_{k+1 / 2}} \int_{y_{j-1 / 2}}^{y_{j+1 / 2}} \int_{x_{i-1 / 2}}^{x_{i+1 / 2}} K^{\prime}(\phi) \times \\
\left(\phi_{i, j, k}-a_{i, j, k}\left(x-x_{i}\right)+b_{i, j, k}\left(y-y_{j}\right)+c_{i, j, k}\left(z-z_{k}\right)+d_{i, j, k}\right)^{2} d x d y d z
\end{aligned}
$$

which in the discrete form is given as

$$
\begin{aligned}
E_{i, j, k}^{\mathrm{CLSVOF}, \Delta}=\sum_{k^{\prime}=k-1}^{k+1} \sum_{j^{\prime}=j-1}^{j+1} \sum_{i^{\prime}=i-1}^{i+1} w_{i^{\prime}-i, j^{\prime}-j, k^{\prime}-k} K_{\epsilon}^{\prime}\left(\phi_{i^{\prime}, j^{\prime}, k^{\prime}}\right) \times \\
\left(\phi_{i^{\prime}, j^{\prime}, k^{\prime}}-a_{i, j, k}\left(x_{i^{\prime}}-x_{i}\right)+b_{i, j, k}\left(y_{j^{\prime}}-y_{j}\right)+c_{i, j, k}\left(z_{k^{\prime}}-z_{k}\right)+d_{i, j, k}\right)^{2}
\end{aligned}
$$

where the weights $w_{i^{\prime}-i, j^{\prime}-j, k^{\prime}-k}$ are chosen such that the Equation A.3 approximates Equation A.2 (similar to that of Sussman and Puckett [17]) and $K_{\epsilon}^{\prime}$ represents a smoothed delta function with thickness $\epsilon=\sqrt{3} \Delta x$ (with $\Delta x=\Delta y=\Delta z$ in our solver) expressed as

$$
K_{\epsilon}^{\prime}(\varphi)=\left\{\begin{array}{l}
\frac{1}{2}\left(1+\cos \left(\frac{\pi \varphi}{\epsilon}\right)\right), \quad \text { if }|\varphi|<\epsilon \\
0, \quad \text { otherwise }
\end{array}\right.
$$

Once the coefficients for the interface are obtained, the value of $d$ is corrected to conserve the liquid volume (upto the machine precision) under reconstructed interface by solving

$$
\left|F^{\text {original }}-F^{\text {reconstructed }}(\boldsymbol{n}, d)\right|=0,
$$


using geometric method [56] where the superscript "original" represent the original liquid volume fraction in the mixed computational cell.

\section{Appendix A.3. Level set correction}

As seen in the Algorithm 8, the level set function at time $t^{n}$ is used for correcting the volume fraction at time $t^{n}$ and subsequently for the next time steps. However, the loss of mass occurred from the level set function is not taken into account in the positioning and orientation of the interface. Moreover, the presence of liquid volume fraction is to reduce such mass loss, hence, it is required to correct the level set function in the vicinity of the interface. The important criterion to keep in mind in designing the correction procedure is to avoid the spurious oscillations in the values of interface curvature. To that end, we have employed the below correction formula for the level set function $\phi$

$$
\phi \leftarrow \omega \phi+(1-\omega) d
$$

where the $\leftarrow$ represents the assignment operator, $d$ is the exact signed distance function, i.e., the shortest distance of the interface from the computational cell center, and $\omega$ is a parameter obtained based on the difference between $\phi$ and $d$ according to Le Chenadec and Pitsch [19].

\section{Appendix A.4. Redistancing level set function}

Due to the correction of the level set function, its signed distance property gets eroded and does not represent the accurate location of the liquid/gas interface anymore. In order to regain the signed distance property, redistancing of this level set function is required and is performed using the method inspired from the work of Sussman et al. 49. The highlight of our redistancing algorithm is that, unlike Sussman et al. [49, we apply it only to the cells which are not corrected by the level set correction algorithm.

\section{Appendix A.5. Volume fraction and level set advection}

A directional splitting advection approach is employed for the transport of the liquid volume fraction $F$ and level set function $\phi$ whose procedure, numerical methods and schemes are explained in Sections 2.1.1 and 2.1.2 the details of which are not recalled here. It is to be noted that this directional splitting advection approach, based on the corrected values of $F$ and $\phi$ computed from the previous steps of the CLSVOF algorithm, involves interface reconstruction after advection along each Cartesian direction.

This algorithm for liquid/gas interface reconstruction within the context of CLSVOF method is coupled with the Navier-Stokes equations for the time advancement of the velocity and pressure fields.

\section{Appendix B. Discretization of centroid advection equation}

This appendix details the numerical method behind the discretization of equation of the advection of the phase centroid (see Equation (22)). The advection of the phase centroid is performed in tandem with that of the liquid volume fraction. The advection equation of the phase centroid (liquid and gas phase centroids) is given as

$$
\frac{\partial \boldsymbol{x}_{\mathrm{COM}}}{\partial t}=\boldsymbol{u}\left(\boldsymbol{x}_{\mathrm{COM}}\right) .
$$

This equation is discretized using Eulerian Implicit-Lagrangian Explicit (EI-LE) scheme.

Now, consider a 2D computational cell shown in Figure B.43 with the velocity on the cell faces, $x$-coordinate of the left and right edges of the cell, and the centroid of liquid and gas phase marked. Before proceeding to the presentation of discretization of Equation (B.1), let $x_{i \pm 1 / 2}=x_{ \pm}$and the subscript "COM" will be dropped hereon (i.e., $\boldsymbol{x}_{\mathrm{COM}}=\boldsymbol{x}$ ). For the sake of simplicity, the subscript "COM" will be dropped for the remainder of this appendix. Without loss of generality, the presentation of the discretization of the advection equation is for the $u$-component of the velocity specifically for the liquid phase centroid advection. The advection along $y$ - and $z$-directions and for the gas phase are fairly straightforward to be 

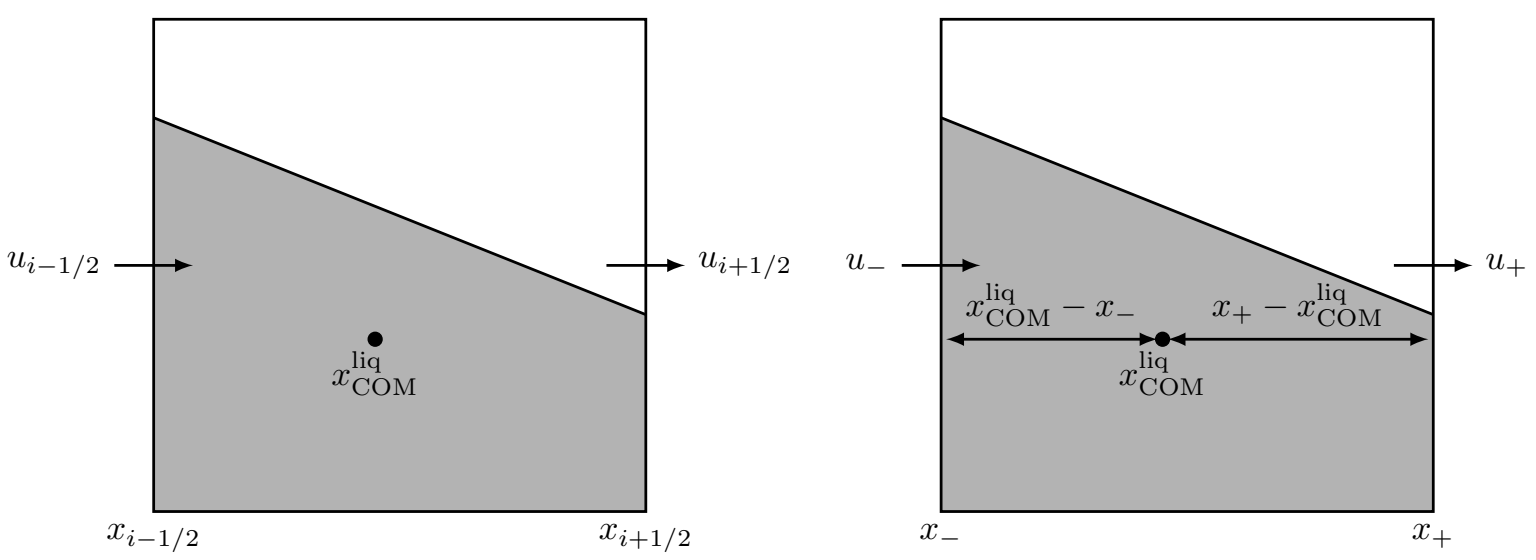

Figure B.43: Illustration of 2D computational cell with liquid centroid for discretization of phase centroid advection equation.

derived similar to that for the liquid phase. Now, discretizing the advection equation using explicit Euler scheme for temporal derivative, we get

$$
\frac{x^{n+1}-x^{n}}{\Delta t}=u(x)
$$

The velocity $u(x)$ is obtained as an interpolated velocity from those at the cell faces according to

$$
u(x)=\frac{u_{i+1 / 2}^{n}\left(x-x_{-}\right)+u_{i-1 / 2}^{n}\left(x_{+}-x\right)}{\Delta x}
$$

Now, substituting Equation $(\bar{B} .3)$ in Equation $(\bar{B} .2)$ and rearranging the terms, we get

$$
x^{n+1}=x^{n}+\frac{u_{i+1 / 2}^{n} \Delta t}{\Delta x}\left(x-x_{-1}\right)+\frac{u_{i-1 / 2}^{n} \Delta t}{\Delta x}\left(x_{+}-x\right)
$$

Now, let $u_{ \pm}^{n}=\frac{u_{i \pm 1 / 2}^{n} \Delta t}{\Delta x}$ which becomes the local cell-based CFL number. Hence, we get,

$$
x^{n+1}=x^{n}+u_{+}^{n}\left(x-x_{-}\right)+u_{-}^{n}\left(x_{+}-x\right),
$$

where $x_{+}, x_{-}$are constants, $u_{ \pm}^{n}$ are taken from time step $t^{n}$. We have two choices for the $x$ to be taken either from time instant $t^{n}$ or $t^{n+1}$ - leading to Lagrangian Explicit (LE) or Eulerian Implicit (EI) schemes, i.e.,

$$
x^{*}=\left\{\begin{array}{l}
x^{n} \Rightarrow \text { Lagrangian Explicit (LE) scheme } \\
x^{n+1} \Rightarrow \text { Eulerian Implicit (EI) scheme }
\end{array}\right.
$$

Appendix B.1. Lagrangian Explicit (LE) scheme: $x=x^{n}$

When the choice is made to take $x^{n}$ for the centroid $x$-coordinate, the equation simplifies to

$$
x^{n+1}=x^{n}+u_{+}\left(x^{n}-x_{-}\right)+u_{-}\left(x_{+}-x^{n}\right) .
$$

Upon grouping the terms that belong to $x^{n}$, we get the final expression

$$
x^{n+1}=L x^{n}-\left(u_{+} x_{-}-u_{-} x_{+}\right),
$$

where $L=1+\left(u_{+}-u_{-}\right)$is the Lagrangian coefficient. 
Appendix B.2. Eulerian Implicit (EI) scheme: $x=x^{n+1}$

With $x=x^{n+1}$ choice for the discretization of the advection equation, we get

$$
x^{n+1}=x^{n}+u_{+}\left(x^{n+1}-x_{-}\right)+u_{-}\left(x_{+}-x^{n+1}\right) .
$$

Now, we group the terms belonging to $x^{n+1}$, we get the final expression as

$$
x^{n+1}=E \times\left(x^{n}-\left(u_{+} x_{-}-u_{-} x_{+}\right)\right)
$$

where $E=1 /\left(1-\left(u_{+}-u_{-}\right)\right)$is the Eulerian coefficient.

In order to be consistent with the liquid volume fraction advection within the framework of directionallysplit advection operations, we switch between the EI and LE schemes between the coordinate directions of advection and also between consecutive time steps in the simulation.

\section{Appendix C. Liquid structure resolution criteria}

This appendix details and derives the interface resolution quality (IRQ) criterion (see Algorithm 3) used within the hybrid moment of fluid-level set (HyMOFLS) framework for tagging cells with moment of fluid (MOF) and coupled level set volume of fluid (CLSVOF) methods of interface reconstruction. In the context of HyMOFLS method, the differentiation between the resolved liquid structures (RLS) and under-resolved liquid structures (URLS) is made using this criterion. The rationale behind the development of HyMOFLS method is to use MOF method for capturing URLS and CLSVOF method for capturing RLS. To that end, let us first consider the expression of IRQ as given in Equation (31),

$$
\operatorname{IRQ}=\frac{1}{\kappa \Delta x},
$$

where $\kappa$ is the local curvature of the interface and $\Delta x$ is the local grid spacing. Albeit the IRQ identifies the resolution of the liquid structure, following the work of Canu [58, the expression in Equation (C.2) is applied to regions of the flow, i.e., it is evaluated in each computational cell containing liquid/gas interface. Hence, Equation (C.2) within the context of ARCHER solver becomes,

$$
\operatorname{IRQ}(i, j, k)=\frac{1}{\kappa(i, j, k) \Delta x}=\left\{\begin{array}{l}
<\alpha, \Rightarrow \text { under-resolved region } \\
\geq \alpha, \Rightarrow \text { resolved region },
\end{array},\right.
$$

where $i, j, k$ are the computational cell indices. Since ARCHER solver has constant mesh spacing, i.e., $\Delta x=\Delta y=\Delta z$ throughout the domain, therefore, the mesh spacing parameter becomes independent of the cell indices. In order to give a context on the IRQ for different computational cells (and regions of the domain), Figure C.44 shows the illustration of an interface topology containing to different curvature regions marked by their respective (approximate constant) radii of curves $R_{1}$ and $R_{2}$. The size of the dashed and dashdotted circles in this figure pertains to these radii values. From the concept of geometry, it is known that curvature is the reciprocal of the radius of the curve, hence, the smaller the radius of the curve, large is the curvature, and hence, according to Equation (C.2) smaller is the IRQ value. When this IRQ value passes below the threshold of $\alpha$, this cell present in this region is termed as the under-resolved region (URR) else it is in the resolved region (RR).

Now, let us derive Equation (C.2) from a geometrical and physical point of view. In a typical atomization simulations, numerous liquid structures of varying sizes and shapes are produced. For the sake of simplicity of the derivation and without loss of generality, consider a sphere (as shown in Figure C.45) as an idealized scenario of the RR and URR presented above in Figure C.44. In mathematical terms, this sphere is set to be under-resolved, when $D<\beta \Delta x$, where $D$ is its diameter. As shown in Equation [30), the $\kappa$ is computed as

$$
\kappa=-\boldsymbol{\nabla} \cdot\left(\frac{\boldsymbol{\nabla} \phi}{\|\boldsymbol{\nabla} \phi\|_{2}}\right)
$$




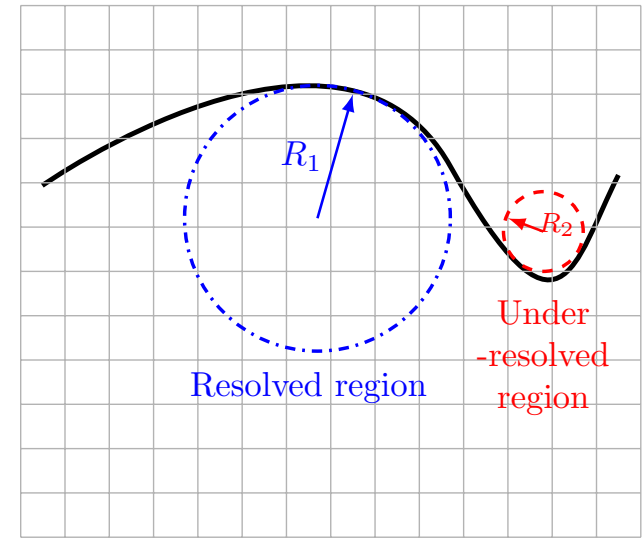

Figure C.44: Radii of curves (approximate constant values) at two regions of interface (black solid line) showing resolved region (RR) and under-resolved region (URR) on an underlying computational grid.

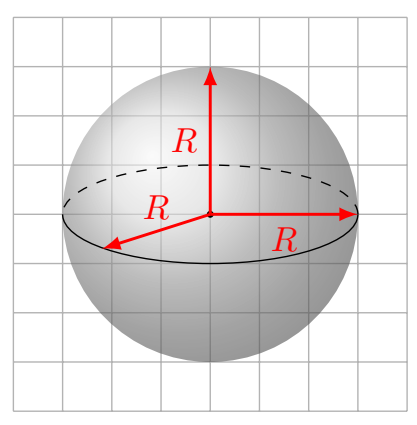

Figure C.45: Radius of sphere for illustration of IRQ.

where $\phi$ is the level set signed distance function. Now, we have

$$
D<\beta \Delta x \Rightarrow \mathrm{URR},
$$

Therefore, $2 R<\beta \Delta x \Rightarrow \mathrm{URR}$,

$\Rightarrow \frac{2 R}{\beta \Delta x}<1 \Rightarrow \mathrm{URR}$,

Therefore, $\frac{2}{\beta} \frac{1}{\frac{1}{R} \Delta x}<1 \Rightarrow$ URR.

Now, from the knowledge of geometry, we know the following relation between the local principal curvatures and local radius of a sphere given as

$$
\kappa_{1}(i, j, k)=\frac{1}{R(i, j, k)} \quad \kappa_{2}(i, j, k)=\frac{1}{R(i, j, k)} .
$$

For the sake of clarity, these indices $i, j, k$ will be dropped hereon for the remainder of this derivation. We also know that the total curvature of an object is computed as $\kappa=\left|\kappa_{1}+\kappa_{2}\right|$. Therefore, we get

$$
\begin{aligned}
& \kappa=\left|\frac{1}{R}+\frac{1}{R}\right|=\left|\frac{2}{R}\right|=\frac{2}{R}(\because R>0), \\
& \text { Therefore, } \frac{\kappa}{2}=\frac{1}{R} .
\end{aligned}
$$

We substitute in this relation into Equation C.7 to get

$$
\begin{aligned}
& \frac{2}{\beta} \frac{1}{\frac{\kappa}{2} \Delta x}<1 \Rightarrow \text { URLS } \\
& \Rightarrow \frac{4}{\beta} \frac{1}{\kappa \Delta x}<1 \Rightarrow \text { URLS } \\
& \frac{1}{\kappa \Delta x}<\frac{\beta}{4} \Rightarrow \text { URLS } \\
& \text { Therefore, IRQ }<\frac{\beta}{4} \Rightarrow \text { URLS } \\
& \text { Therefore, IRQ }<\alpha \Rightarrow \text { URLS }
\end{aligned}
$$


where $\alpha=\beta / 4$. In this work, we choose $\beta=8$ thereby obtaining $\alpha=2$. The value of $\beta=8$ corresponds to the scenario, when there are 8 or less than 8 computational cells spanning the diameter of the sphere, it is considered under-resolved region otherwise resolved region [58]. Therefore, the final under-resolved liquid structure/resolved liquid structure (URLS/RLS) criterion for distinguishing the derivation of the criterion for detecting URLS arrives to the following condition,

$$
\operatorname{IRQ}=\frac{1}{\kappa \Delta x}=\left\{\begin{array}{l}
<2, \Rightarrow \text { Under-resolved structure } \\
\geq 2, \Rightarrow \text { Resolved liquid structure }
\end{array}\right.
$$

Since the value of IRQ is computed from the local curvature $\kappa$ and the local mesh spacing $\Delta x$, the URLS/RLS criterion is checked in every computational cell that contains liquid structure, i.e., liquid/gas interface. This means that for each computational cell containing a non-zero value of liquid volume fraction (deeming it belonging to a liquid structure), the IRQ value is computed based on local $\kappa$ and local $\Delta x$ in that cell. The computed value for IRQ is then checked for the URLS/RLS criterion (Equation (C.11) for the purposes of tagging cells with MOF or CLSVOF method within the context of HyMOFLS method of interface reconstruction.

\section{References}

[1] J. Anez, A. Ahmed, N. Hecht, J. Reveillon, F.-X. Demoulin, Eulerian-lagrangian spray atomization model coupled with interface capturing method for diesel injectors, International Journal of Multiphase Flow 113 (2019) 325-342. URL: https://doi.org/10.1016/j.ijmultiphaseflow.2018.10.009 doi 10.1016/j.ijmultiphaseflow.2018.10.009

[2] M. Gorokhovski, M. Herrmann, Modeling primary atomization, Annual Review of Fluid Mechanics 40 (2008) $343-366$. URL: https://doi.org/10.1146/annurev.fluid.40.111406.102200 doi 10.1146/annurev.fluid.40.111406.102200

[3] J. U. Brackbill, D. B. Kothe, C. Zemach, A continuum method for modeling surface tension 100 (1992) 335-354. URL: https://doi.org/10.1016/0021-9991(92)90240-Y doi 10.1016/0021-9991(92)90240-Y

[4] R. Fedkiw, T. Aslam, B. Merriman, S. Osher, A Non-oscillatory Eulerian Approach to Interfaces in Multimaterial Flows (the Ghost Fluid Method), Journal of Computational Physics 152 (1999) 457-492. URL: https://doi.org/10.1006/jcph. 1999.6236 doi $10.1006 / \mathrm{jcph} .1999 .6236$.

[5] E. Aulisa, S. Manservisi, R. Scardovelli, S. Zaleski, A geometrical area-preserving Volume-of-Fluid advection method, Journal of Computational Physics 192 (2003) 355-364. URL:https://doi.org/10.1016/j.jcp.2003.07.003 doi 10.1016/ j.jcp.2003.07.003

[6] J. Hernández, J. López, P. Gómez, C. Zanzi, F. Faura, A new volume of fluid method in three dimensions-Part I: Multidimensional advection method with face-matched flux polyhedra, International Journal for Numerical Methods in Fluids 58 (2008) 897-921. URL: https://doi.org/10.1002/fld.1776 doi 10.1002/fld.1776

[7] J. López, C. Zanzi, P. Gómez, F. Faura, J. Hernández, A new volume of fluid method in three dimensions-Part II: Piecewise-planar interface reconstruction with cubic-Bézier fit, International Journal for Numerical Methods in Fluids 58 (2008) 923-944. URL: https://doi.org/10.1002/fld.1775 doi 10.1002/fld.1775

[8] S. Osher, J. A. Sethian, Fronts propagating with curvature-dependent speed: Algorithms based on Hamilton-Jacobi formulations, Journal of Computational Physics 79 (1988) 12-49. URL: https://doi.org/10.1016/0021-9991(88)90002-2 doi $10.1016 / 0021-9991(88) 90002-2$

[9] S. Osher, R. Fedkiw, Level Set Methods and Dynamic Implicit Surfaces, Springer, 2003.

[10] O. Desjardins, V. Moureau, H. Pitsch, An accurate conservative level set/ghost fluid method for simulating turbulent atomization, Journal of Computational Physics 227 (2008) 8395-8416. URL: https://doi.org/10.1016/j.jcp.2008.05. 027. doi $10.1016 / j \cdot j c p .2008 .05 .027$.

[11] E. Aulisa, S. Manservisi, R. Scardovelli, S. Zaleski, Interface reconstruction with least-squares fit and split advection in three-dimensional cartesian geometry, Journal of Computational Physics 225 (2007) 2301-2319. URL: https://doi.org/ $10.1016 / j \cdot j c p .2007 .03 .015$ doi $10.1016 / j \cdot j c p .2007 .03 .015$

[12] P. Cifani, W. R. Michalek, G. J. M. Priems, J. G. M. Kuerten, C. W. M. van der Geld, B. J. Geurts, A comparison between the surface compression method and an interface reconstruction method for the VOF approach, Computers \& Fluids 136 (2016) 421-435. URL: https://doi.org/10.1016/j.compfluid.2016.06.026 doi 10.1016/j.compfluid.2016.06.026

[13] H. Grosshans, R.-B. Szász, L. Fuchs, Development of a Combined VOF-LPT Method to Simulate Two-phase Flows in Various Regimes, in: Proceedings of the 7th International Symposium on Turbulence and Shear Flow Phenomena, TSFP7, July 28-31, Ottawa, Canada, 2011.

[14] A. Orazzo, I. Lagrange, J.-L. Estivalézes, D. Zuzio, A VoF-Based Consistent Mass-Momentum Transport for TwoPhase Flow Simulations, in: Proceedings of the ASME 17th International Symposium on Numerical Methods for Multiphase in FEDSM2017, July 30-August 3, 2017, Waikoloa, HI, USA, 2017, pp. 1-11. URL: https://doi.org/10.1115/ FEDSM2017-69190 doi 10.1115/FEDSM2017-69190

[15] E. Olsson, G. Kreiss, A conservative level set method for two phase flow, Journal of Computational Physics 210 (2005) 225-246. URL: https://doi.org/10.1016/j.jcp.2005.04.007 doi 10.1016/j.jcp.2005.04.007 
[16] R. Chiodi, O. Desjardins, A reformulation of the conservative level set reinitialization equation for accurate and robust simulation of complex multiphase flows, Journal of Computational Physics 343 (2017) 186-200. URL: https://doi.org/ $10.1016 / j \cdot j c p .2017 .04 .053$ doi $10.1016 / j \cdot j c p .2017 .04 .053$

[17] M. Sussman, E. G. Puckett, A Coupled Level Set and Volume-of-Fluid Method for Computing 3D and Axisymmetric Incompressible Two-Phase Flows, Journal of Computational Physics 162, (2000) 301-337. URL: https://doi.org/10. 1006/jcph.2000.6537 doi $10.1006 /$ jcph.2000.6537.

[18] T. Ménard, S. Tanguy, A. Berlemont, Coupling level set/VOF/ghost fluid methods: Validation and application to 3D simulation of the primary break-up of a liquid jet, International Journal of Multiphase Flow 33 (2007) 510-524. URL: https://doi.org/10.1016/j.ijmultiphaseflow.2006.11.001 doi 10.1016/j.ijmultiphaseflow.2006.11.001

[19] V. Le Chenadec, H. Pitsch, A 3D Unsplit Forward/Backward Volume-of-Fluid Approach and Coupling to the Level Set Method, Journal of Computational Physics 233 (2013) 10-33. URL: https://doi.org/10.1016/j.jcp.2012.07.019 doi $10.1016 / j \cdot j c p .2012 .07 .019$

[20] M. Herrmann, A balanced force refined level set grid method for two-phase flows on unstructured flow solver grids, Journal of Computational Physics 227 (2008) 2674-2706. URL: https://doi.org/10.1016/j.jcp.2007.11.002. doi 10. $1016 / j \cdot j \mathrm{jcp} .2007 .11 .002$

[21] O. Desjardins, H. Pitsch, A spectrally refined interface approach for simulating multiphase flows, Journal of Computational Physics 228 (2009) 1658-1677. URL: https://doi.org/10.1016/j.jcp.2008.11.005 doi 10.1016/j.jcp.2008.11.005

[22] V. Dyadechko, M. Shashkov, Reconstruction of multi-material interfaces from moment data, Journal of Computational Physics 227 (2008) 5361-5384. URL: https://doi.org/10.1016/j.jcp.2007.12.029 doi 10.1016/j.jcp.2007.12.029

[23] H. T. Ahn, M. Shashkov, Adaptive Moment-of-Fluid Method, Technical Report LA-UR-08-2153, Los Alamos National Laboratory, 2008

[24] H. T. Ahn, M. Shashkov, Adaptive moment-of-fluid method, Journal of Computational Physics 228 (2009) $2792-2821$. URL: https://doi.org/10.1016/j·jcp.2008.12.031 doi 10.1016/j·jcp.2008.12.031

[25] H. T. Ahn, M. Shashkov, M. A. Christon, The moment-of-fluid method in action, Communications in Numerical Methods in Engineering 25 (2009) 1009-1018. doi 10.1002/cnm.1135

[26] R. N. Hill, M. Shashkov, The symmetric moment-of-fluid interface reconstruction algorithm, Journal of Computational Physics 249 (2013) 180-184. URL: https://doi.org/10.1016/j.jcp.2013.04.037 doi 10.1016/j.jcp.2013.04.037

[27] M. Jemison, E. Loch, M. Sussman, M. Shashkov, M. Arienti, M. Ohta, Y. Wang, A Coupled Level Set-Moment of Fluid Method for Incompressible Two-Phase Flows, Journal of Scientific Computing 54 (2013) 454-491. URL: https: //doi.org/10.1007/s10915-012-9614-7 doi 10.1007/s10915-012-9614-7

[28] X. Chen, X. Zhang, An improved 3d mof method based on analytical partial derivatives, Journal of Computational Physics 326 (2016) 156-170. URL: http://dx.doi.org/10.1016/j.jcp.2016.08.051 doi 10.1016/j.jcp.2016.08.051

[29] X. Chen, X. Zhang, An improved 2D MoF method by using high order derivatives, Journal of Computational Physics 349 (2017) 176-190. URL: http://dx.doi.org/10.1016/j.jcp.2017.08.031 doi 10.1016/j.jcp.2017.08.031

[30] A. Lemoine, S. Glockner, J. Breil, Moment-of-fluid analytic reconstruction on 2D Cartesian grids, Journal of Computational Physics 328 (2017) 131-139. URL: https://doi.org/10.1016/j.jcp.2016.10.013 doi 10.1016/j.jcp.2016.10.013

[31] T. Milcent, A. Lemoine, Moment-of-fluid analytic reconstruction on 3D rectangular hexahedrons 409 (2020) 109346. URL: https://doi.org/10.1016/j.jcp.2020.109346 doi 10.1016/j.jcp.2020.109346

[32] A. Lemoine, Analytic gradient for the moment-of-fluid method in axisymmetric and on general polyhedrons in any dimension 422 (2020). URL: https://doi.org/10.1016/j.jcp.2020.109741 doi 10.1016/j.jcp.2020.109741.

[33] M. Jemison, M. Sussman, M. Arienti, Compressible, multiphase semi-implicit method with moment of fluid interface representation, Journal of Computational Physics 279 (2014) 182-217.

[34] M. Jemison, M. Sussman, M. Shashkov, Filament capturing with the multimaterial moment-of-fluid method, Journal of Computational Physics 285 (2015) 149-172.

[35] R. Chiodi, O. Desjardins, A two-plane interface reconstruction strategy to enable thin-films in vof simulations, in: Proceedings of the ICLASS, 14th Triennial International Conference on Liquid Atomization and Spray Systems, July 22-26, Chicago, IL, USA, 2018.

[36] H. Anbarlooei, K. Mazaheri, Moment of fluid interface reconstruction method in multi-material arbitrary lagrangian eulerian (mmale) algorithms, Computer Methods in Applied Mechanics and Engineering 198 (2009) 3782-3794. URL: https://doi.org/10.1016/j.cma.2009.08.009 doi 10.1016/j.cma.2009.08.009

[37] M. Kucharik, R. V. Garimella, S. P. Schofield, M. Shashkov, A comparative study of interface reconstruction methods for multi-material ale simulations, Journal of Computational Physics 229 (2010) 2432-2452. URL: https://doi.org/10. $1016 / j \cdot j c p .2009 .07 .009$ doi $10.1016 / j \cdot j c p .2009 .07 .009$

[38] H. R. Anbarlooei, K. Mazaheri, Moment of fluid interface reconstruction method in axisymmetric coordinates, International Journal for Numerical Methods in Biomedical Engineering 27 (2011) 1640-1651. doi 10.1002/cnm.1426

[39] S. Galera, J. Breil, P.-H. Maire, A 2D unstructured multi-material Cell-Centered Arbitrary Lagrangian-Eulerian (CCALE) scheme using MOF interface reconstruction, Computers \& Fluids 46 (2011) 237-244. URL: https://doi.org/10.1016/j. compfluid.2010.09.038 doi 10.1016/j.compfluid.2010.09.038

[40] S. P. Schofield, , M. A. Christon, Effects of element order and interface reconstruction in FEM/volume-of-fluid incompressible flow simulation, International Journal for Numerical Methods in Fluids 68 (2012) 1422-1437. doi 10.1002/fld.3657

[41] J. Breil, T. Harribey, P.-H. Maire, M. Shashkov, A multi-material ReALE method with MOF interface reconstruction, Computers \& Fluids 83 (2013) 115-125. URL: http://dx.doi.org/10.1016/j.compfluid.2012.08.015 doi 10.1016/j. compfluid.2012.08.015

[42] M. B. Freiss, J. Breil, P.-H. Maire, M. Shashkov, A Multi-Material CCALE-MOF Approach in Cylindrical Geometry, Communications in Computational Physics 15 (2014) 330-364. URL: https://doi.org/10.4208/cicp.190912.080513a 
doi $10.4208 /$ cicp. 190912.080513a

[43] G. Li, Y. Lian, Y. Guo, M. Jemison, M. Sussman, T. Helms, M. Arienti, Incompressible multiphase flow and encapsulation simulations using the moment-of-fluid method, International Journal for Numerical Methods in Fluids 79 (2015) 456-490. URL: https://doi.org/10.1002/fld.4062 doi 10.1002/fld.4062

[44] H. K. Zinjala, J. Banerjee, A lagrangian-eulerian advection scheme with moment-of-fluid interface reconstruction, Numerical Heat Transfer, Part B: Fundamentals 69 (2016) 563-574. URL: http://dx.doi.org/10.1080/10407790.2016.1138753 doi $10.1080 / 10407790.2016 .1138753$

[45] A. Asuri Mukundan, T. Ménard, J. C. Brändle de Motta, A. Berlemont, A 3D Moment of Fluid method for simulating complex turbulent multiphase flows, Computers \& Fluids 198 (2020). URL: https://doi.org/10.1016/j.compfluid. 2019.104364 doi $10.1016 / \mathrm{j}$. compfluid.2019.104364

[46] A. Asuri Mukundan, Numerical development of Moment of Fluid/Level Set method and Application to liquid jet and sheet atomization, Ph.D. thesis, Université de Rouen Normandie, 2020.

[47] R. Canu, B. Duret, J. Reveillon, F.-X. Demoulin, Curvature-based interface resolution quality (IRQ) indicator to assess simulation accuracy 30 (2020) 31-53. doi 10.1615/AtomizSpr.2020033923

[48] G. Vaudor, T. Ménard, W. Aniszewski, M. Doring, A. Berlemont, A consistent mass and momentum flux computation method for two phase flows. Application to atomization process, Computers \& Fluids 152 (2017) 204-216. URL: https: //doi.org/10.1016/j.compfluid.2017.04.023 doi 10.1016/j.compfluid.2017.04.023

[49] M. Sussman, E. Fatemi, P. Smereka, S. Osher, An improved level set method for incompressible two-phase flows, Computers \& Fluids 27 (1998) 663-680. URL: https://doi.org/10.1016/S0045-7930(97)00053-4 doi 10.1016/S0045-7930(97) 00053-4

[50] M. Sussman, A. S. Almgren, J. B. Bell, P. Colella, L. H. Howell, M. L. Welcome, An adaptive level set approach for incompressible two-phase flows, Journal of Computational Physics 148 (1999) 81-124. URL: https://doi.org/10.1006/ jcph.1998.6106 doi $10.1006 / j \mathrm{jph} .1998 .6106$

[51] G. D. Weymouth, D. K.-P. Yue, Conservative Volume-of-Fluid method for free-surface simulations on Cartesian-grids, Journal of Computational Physics 229 (2010) 2853-2865. URL: https://doi.org/10.1016/j.jcp.2009.12.018 doi 10 . $1016 / j \cdot j c p .2009 .12 .018$

[52] R. Scardovelli, S. Zaleski, Interface reconstruction with least-square fit and split eulerian-lagrangian advection, International Journal for Numerical Methods in Fluids 41 (2003) 251-274. URL: https://doi.org/10.1002/fld.431 doi $10.1002 /$ fld.431

[53] J. Cousin, T. Ménard, A. Berlemont, S. Grout, Primary breakup simulation of a liquid jet discharged by a low-pressure compound nozzle, Computers \& Fluids 63 (2012) 165-173. URL: http://dx.doi.org/10.1016/j.compfluid.2012.04.013 doi $10.1016 / j$.compfluid.2012.04.013

[54] B. Duret, J. Réveillon, T. Ménard, F. X. Demoulin, Improving primary atomization modeling through DNS of two-phase flows, International Journal of Multiphase Flow 55 (2013) 130-137. URL: https://doi.org/10.1016/j.ijmultiphaseflow. 2013.05.004 doi 10.1016/j.ijmultiphaseflow.2013.05.004

[55] H. T. Ahn, M. Shashkov, Multi-material interface reconstruction on generalized polyhedral meshes, Journal of Computational Physics 226 (2007) 2096-2132. URL: https://doi.org/10.1016/j.jcp.2007.06.033 doi 10.1016/j.jcp.2007.06. 033 .

[56] D. Gueyffier, A. Nadim, R. Scardovelli, S. Zaleski, Volume-of-Fluid Interface Tracking with Smoothed Surface Stress Methods for Three-Dimensional Flows, Journal of Computational Physics 152 (1999) 423-456. URL: https://doi.org/ $10.1006 / \mathrm{jcph} .1998 .6168$ doi $10.1006 / \mathrm{jcph} .1998 .6168$

[57] G. Kindlmann, R. Whitaker, T. Rasdizen, T. Möller, Curvature-based transfer functions for direct volume rendering: Methods and applications, in: Proceedings of the Visualization, VIS 2003. IEEE, 2003. doi 10.1109/VISUAL.2003.1250414

[58] R. Canu, Développement d'une méthode compressible avec évaporation pour la simulation d'interface résolue dans le cadre de l'atomisation, Ph.D. thesis, Université de Rouen Normandie, 2019.

[59] K. E. Wardle, H. G. Weller, Hybrid Multiphase CFD Solver for Coupled Dispersed/Segregated Flows in Liquid-Liquid Extraction, International Journal of Chemical Engineering 2013 (2013) 1-13. URL: https://doi.org/10.1155/2013/ 128936 doi $10.1155 / 2013 / 128936$

[60] Utilisation du Cluster Myria, CRIANN Supercomputing Facility, Centre Régional Informatique et d'Applications Numériques de Normandie, 2018. URL: http://www-tech.criann.fr/calcul/tech/myria-doc/guide-util/

[61] D. Enright, R. Fedkiw, J. Ferziger, I. Mitchell, A Hybrid Particle Level Set Method for Improved Interface Capturing, Journal of Computational Physics 183 (2002) 83-116. URL: https://doi.org/10.1006/jcph.2002.7166 doi 10.1006/ jcph.2002.7166

[62] R. J. LeVeque, High-Resolution Conservative Algorithms for Advection in Incompressible Flow, SIAM Journal on Numerical Analysis 33 (1996) 627-665. URL: https://doi.org/10.1137/0733033 doi $10.1137 / 0733033$

[63] B. Duret, G. Luret, J. Réveillon, T. Ménard, A. Berlemont, F.-X. Demoulin, DNS analysis of turbulent mixing in two-phase flows, International Journal of Multiphase Flow 40 (2012) 93-105. URL: https://doi.org/10.1016/j.ijmultiphaseflow. 2011.11.014 doi 10.1016/j.ijmultiphaseflow.2011.11.014

[64] R. Canu, S. Puggelli, M. Essadiki, B. Duret, T. Ménard, M. Massot, J. Réveillon, F. Demoulin, Where does the droplet size distribution come from?, International Journal of Multiphase Flow 107 (2018) 230-245. URL: https://doi.org/10. 1016/j.ijmultiphaseflow.2018.06.010 doi 10.1016/j.ijmultiphaseflow.2018.06.010

[65] V. Chéron, J. C. Brändle de Motta, G. Vaudor, T. Ménard, A. Berlemont, From droplets to particles: Transformation criteria, in: Proceedings of the ILASS Europe, 29th Annual Conference on Liquid Atomization and Spray Systems, September 2-4, Paris, France, 2019.

[66] S. Tanguy, A. Berlemont, Application of a level set method for simulation of droplet collisions, International Jour- 
nal of Multiphase Flow 31 (2005) 1015-1035. URL: https://10.1016/j.ijmultiphaseflow.2005.05.010 doi 10.1016/j. ijmultiphaseflow.2005.05.010

[67] M. Klein, A. Sadiki, J. Janicka, A digital filter based generation of inflow data for spatially developing direct numerical or large eddy simulations, Journal of Computational Physics 186 (2003) 652-665. URL: https://doi.org/10.1016/ S0021-9991(03)00090-1 doi 10.1016/S0021-9991(03)00090-1

[68] M. Sussman, K. M. Smith, M. Y. Hussaini, M. Ohta, R. Zhi-Wei, A sharp interface method for incompressible twophase flows, Journal of Computational Physics 221 (2007) 469-505. URL: https://doi.org/10.1016/j.jcp.2006.06.020 doi $10.1016 / j \cdot j c p .2006 .06 .020$

[69] M. Kang, R. Fedkiw, X. D. Liu, A Boundary Condition Capturing Method for Multiphase Incompressible Flow, Journal of Scientific Computing 15 (2000) 323-360. URL: https://doi.org/10.1023/A:1011178417620 doi 10.1023/A: 1011178417620

[70] N. Ashgriz, J. Poo, Coalescence and separation in binary collisions of liquid drops, Journal of Fluid Mechanics 221 (1990) 183-204. URL: https://doi.org/10.1017/S0022112090003536 doi 10.1017/S0022112090003536

[71] A. Prosperetti, Viscous effects on small-amplitude surface waves, Physics of Fluids 19 (1976) 195-203. URL: https: //doi.org/10.1063/1.861446 doi 10.1063/1.861446

[72] R. Menikoff, R. C. Mjolsness, D. H. Sharp, C. Zemach, B. J. Doyle, Initial value problem for Rayleigh-Taylor instability of viscous fluids, Physics of Fluids 21 (1978) 1674-1687. URL: https://doi.org/10.1063/1.862107 doi 10.1063/1.862107

[73] A. Prosperetti, Motion of two superposed viscous fluids, Physics of Fluids 24 (1981) 1217-1223. URL: https://doi.org/ 10.1063/1.863522 doi 10.1063/1.863522

[74] C. Weber, Disintegration of liquid jets, Zeitschrift für Angewandte Mathematik und Mechanik 11 (1931) 136-154.

[75] M. Herrmann, A parallel Eulerian interface tracking/Lagrangian point particle multi-scale coupling procedure, Journal of Computational Physics 229 (2010) 745-759.

[76] K. Warncke, S. Gepperth, B. Sauer, A. Sadiki, J. Janicka, R. Koch, H.-J. Bauer, Experimental and numerical investigation of the primary breakup of an airblasted liquid sheet, International Journal of Multiphase Flow 91 (2017) 208-224. URL: http://dx.doi.org/10.1016/j.ijmultiphaseflow.2016.12.010 doi 10.1016/j.ijmultiphaseflow.2016.12.010

[77] C. Dumouchel, Liquid Atomization and Spray: A Multi-Scale Description, in: Proceedings of the ASME 2017 Fluids Engineering Division Summer Meeting, FEDSM2017, July 30-August 3, Waikoloa, HI, USA, 2017. URL: https://doi.org/10.1115/FEDSM2017-69590 doi 10.1115/FEDSM2017-69590 arXiv:https://hal-normandie-univ.archives-ouvertes.fr/hal-01621280/document

[78] S. Gepperth, A. Müller, R. Koch, H.-J. Bauer, Ligament and droplet characteristics in prefilming airblast atomization, in: Proceedings of the ICLASS, 12th Triennial International Conference on Liquid Atomization and Spray Systems, September 2-6, Heidelberg, Germany, 2012. 